\title{
Asymptotic Behavior of Stable Structures Made of Beams
}

\author{
Georges Griso $^{1}$ - Larysa Khilkova ${ }^{2} \cdot$ Julia Orlik $^{2}$. \\ Olena Sivak ${ }^{2}$
}

Received: 24 July 2020 / Accepted: 12 January 2021 / Published online: 5 February 2021

(C) The Author(s) 2021

\begin{abstract}
In this paper, we study the asymptotic behavior of an $\varepsilon$-periodic 3D stable structure made of beams of circular cross-section of radius $r$ when the periodicity parameter $\varepsilon$ and the ratio $r / \varepsilon$ simultaneously tend to 0 . The analysis is performed within the frame of linear elasticity theory and it is based on the known decomposition of the beam displacements into a beam centerline displacement, a small rotation of the cross-sections and a warping (the deformation of the cross-sections). This decomposition allows to obtain Korn type inequalities. We introduce two unfolding operators, one for the homogenization of the set of beam centerlines and another for the dimension reduction of the beams. The limit homogenized problem is still a linear elastic, second order PDE.
\end{abstract}

Keywords Linear elasticity · Homogenization - Stable structure - Periodic beam structure · Periodic unfolding method $\cdot$ Dimension reduction $\cdot$ Korn inequalities

Mathematics Subject Classification (2010) 35B27 · 35J50 • 47H05 · 74B05 · 74K10 • $74 \mathrm{~K} 20$

\section{Introduction}

The aim of this work is to study the asymptotic behavior of an $\varepsilon$-periodic 3D stable structure made of "thin" beams of circular cross-section of radius $r$ when the periodicity parameter $\varepsilon$

J. Orlik

julia.orlik@itwm.fraunhofer.de

G. Griso

griso@1j11.math.upmc.fr

L. Khilkova

LarisaHilkova@gmail.com

O. Sivak

olena.sivak@itwm.fraunhofer.de

1 Sorbonne Université, CNRS, Université de Paris, Laboratoire Jacques-Louis Lions (LJLL), F-75005 Paris, France

2 Department SMS, Fraunhofer ITWM, 1 Fraunhofer Platz, 67663 Kaiserslautern, Germany 
tends to 0 , in the framework of the linear elasticity. By "thin", we mean that the radius $r$ of the beams is much smaller than the periodicity parameter $\varepsilon$ and that we deal with the case where $\varepsilon$ and $r / \varepsilon$ simultaneously tend to 0 .

It is well known to engineers that for wire trusses, lattices made of very thin beams, bending dominates the stretching-compression. A contrario, if the same structures are made of thick beams the stretching-compression dominates. This is what several mathematical studies of recent decades have obtained for periodic structures made of beams. For such structures, from the mathematical point of view, this means that the processes of homogenization and dimension reduction do not commute (see the pioneer works $[5,11,12]$ and also $[1,6,8,24,25,27,28,31])$. Our aim is to investigate between these extreme cases. More precisely, we consider the case for which the ratios $\operatorname{diam}(\Omega) / \varepsilon$ and $\varepsilon / r$ are of the same order ( $\Omega$ is the $3 D$ domain covered by the beam structure). In Sects. 5 and following, we show that the ratio $r / \varepsilon^{2}$ and its limit $\kappa \in[0,+\infty]$ play an important role in the estimates and the asymptotic behaviors. It worth to notice that in our analysis, $\kappa=0$ also corresponds to the case where first the dimension reduction is done and then the homogenization, while $\kappa=+\infty$ is for the vice-versa case. In the convergences (7.12) of Theorem 2, we show that the rescaled global displacement depends on $\kappa$. If $\kappa \in(0,+\infty)$, its limit is a combination of a global displacement (a pure stretching-compression) and a local bending; if $\kappa=+\infty$ it is just a global displacement and if $\kappa=0$ it is a local bending.

Our analysis relies on a displacement decomposition for a single beam introduced in [13-15]. According to those studies, a beam displacement is the sum of an elementary displacement and a warping. The elementary displacement has two components. The first one is the displacement of the beam centerline while the second stands for the small rotation of the beam cross-sections (see $[13,15]$ ). This decomposition has been extended for structures made of a large number of beams in [14] (see [4] for the structures made of beams in the nonlinear elasticity framework). Here, similar displacement decompositions are obtained, these decompositions are used for stable beam structures (see Lemma 5) and then for periodic 3D stable structures made of beams. It is important to note that estimate $(4.5)_{1}$ is the key point of this paper. It characterizes the stable structures. In a forthcoming paper, we will investigate the unstable and auxetic 3D periodic structures made of beams and we will see that all the estimates of Lemma 5 will remain except (4.5) 1 . These decompositions allow to obtain Korn type inequalities as well as relevant estimates of the centerline displacements.

To study the asymptotic behavior of periodic stable structures and derive limit problem we use the periodic unfolding method introduced in [9] and then developed in [10]. This method has been applied to a large number of different types of problems. We mention only a few of them which deal with periodic structures in the framework of the linear elasticity (see $[3,16-21,26])$. As general references on the theory of beams or structures made of beams, we refer to $[2,7,22,23,29,30]$.

The paper is organized as follows. Section 2 introduces structures made of segments and remind properties of Sobolev spaces defined on these structures. Furthermore, in this section we give a simple definition of stable and unstable structures and present several examples. In Sect. 3 we remind known results concerning the decomposition of a beam displacement into an elementary displacement and a warping. This section also gives estimates with respect to the $L^{2}$-norm of the strain tensor of the terms appearing in the decomposition. In Sect. 4 we extend the results of the previous section to structures made of beams. Complete estimates of our decomposition terms and Korn-type inequalities are obtained for stable structures.

In Sect. 5 we deal with an $\varepsilon$-periodic stable 3D structure made of $r$-thin beams, $\mathcal{S}_{\varepsilon, r}$. For this structure we introduce a linearized elasticity problem and specify the assumptions on the applied forces. Using results from the previous section we decompose every displacement 
of $\mathcal{S}_{\varepsilon, r}$ as the sum of an elementary displacement and a warping and provide estimates of the terms of this decomposition. The scaling of the applied forces are given with respect to $\varepsilon$ and $r$. That leads to an upper bound for the $L^{2}$-norm of the strain tensor of the solution of the elasticity problem of order 1 .

In Sect. 6 we introduce different types of unfolding operators, mainly one for the centerline beams and another for the cross-sections. This last operator concerns the dimension reduction. Several results on these operators are given in this section and Appendix C.

Sect. 7, deals with the asymptotic behavior of a sequence of displacements and their strain tensors. Then, in Sect. 8, in order to obtain the limit unfolded problem we split it into three problems: the first involving the limit warpings (these fields are concentrated in the cross-sections, this step corresponds mainly to the process of dimension reduction), the second involving the local extensional and inextensional limit displacements posed on the skeleton structure and the third involving the macroscopic limit displacement posed in the homogeneous domain $\Omega$.

In Sect. 9 we complete this analysis by giving the homogenized limit problem (Theorem 4). We obtain a linear elasticity problem with constant coefficients calculated using the correctors.

In Sect. 10 we apply the previously obtained results in the case where the periodic 3D beam structure is made of isotropic and homogeneous material. We present an approximation to the solution of the linearized elasticity problem which can be explicitly computed using the solution of the homogenized problem.

In the Appendix we give the most technical results.

\section{Geometric Setting}

\subsection{Structures Made of Segments}

In this paper we consider structures made up of a large number of segments.

Definition 1 Let $\mathcal{S}=\bigcup_{\ell=1}^{m} \gamma_{\ell}, \gamma_{\ell} \doteq\left[\mathbf{A}^{\ell}, \mathbf{B}^{\ell}\right]$, be a set of segments and $\mathcal{K}$ the set of the extremities of these segments.

\section{$\mathcal{S}$ is a structure if}

- $\mathcal{S}$ is nonincluded in a plane,

- $\mathcal{S}$ is connected,

- a common point to two segments is a common extremity of these segments,

- if an element of $\mathcal{K}$ belongs to only two segments then the directions of these segments are noncollinear,

- for every segment $\gamma_{\ell}$ we denote $\mathbf{t}_{1}^{\ell}$ a unit vector in the direction of $\gamma_{\ell}, \ell \in\{1, \ldots, m\}$.

We denote $\mathbf{t}_{1}$ the field belonging to $L^{\infty}(\mathcal{S})^{3}$ defined by

$$
\mathbf{t}_{1}=\mathbf{t}_{1}^{\ell} \quad \text { a.e. in } \gamma_{\ell}, \quad \ell \in\{1, \ldots, m\} .
$$

The segment $\gamma_{\ell} \subset \mathcal{S}$ of length $l_{\ell}$ is parameterized by $S_{1} \in\left[0, l_{\ell}\right], \ell \in\{1, \ldots, m\}$

$$
\gamma_{\ell} \doteq\left[\mathbf{A}^{\ell}, \mathbf{B}^{\ell}\right] \doteq\left\{\mathbf{A}^{\ell}+S_{1} \mathbf{t}_{1}^{\ell} \in \mathbb{R}^{3} \mid \quad S_{1} \in\left[0, l_{\ell}\right]\right\}, \quad\left(\mathbf{A}^{\ell}, \mathbf{B}^{\ell}\right) \in \mathcal{K}^{2}
$$

The running point of $\mathcal{S}$ is denoted $\mathbf{S}$. For all $\mathbf{S} \in \gamma_{\ell}$ one has $\mathbf{S}=\mathbf{A}^{\ell}+S_{1} \mathbf{t}_{1}^{\ell}, S_{1} \in\left[0, l_{\ell}\right]$, $\ell \in\{1, \ldots, m\}$. 


\subsection{Some Reminders on the Sobolev Spaces $L^{p}(\mathcal{S})$ and $H^{1}(\mathcal{S})$}

A measurable function $\Phi$ defined on $\mathcal{S}$ belongs to $L^{p}(\mathcal{S}), p \in[1,+\infty]$, if for every segment $\gamma_{\ell} \subset \mathcal{S}$, one has $\Phi_{\mid \gamma_{\ell}} \in L^{p}\left(\gamma_{\ell}\right), \ell \in\{1, \ldots, m\}$.

For every $\Phi \in L^{1}(\mathcal{S})$ define

$$
\int_{\mathcal{S}} \Phi(\mathbf{S}) d \mathbf{S} \doteq \sum_{\ell=1}^{m} \int_{0}^{l_{\ell}} \Phi\left(\mathbf{A}^{\ell}+S_{1} \mathbf{t}_{1}^{\ell}\right) d S_{1} .
$$

Observe that the right-hand side of the above equality does not depend on the choice of a unit vector in the directions of the segments. The space $L^{2}(\mathcal{S})$ is endowed with the norm

$$
\|\psi\|_{L^{2}(\mathcal{S})} \doteq \sqrt{\int_{\mathcal{S}}|\psi(\mathbf{S})|^{2} d \mathbf{S},} \quad \forall \psi \in L^{2}(\mathcal{S}) .
$$

Set

$$
H^{1}(\mathcal{S}) \doteq\left\{\psi \in C(\mathcal{S}) \mid \psi_{\mid \gamma_{\ell}} \in H^{1}\left(\gamma_{\ell}\right), \quad \ell \in\{1, \ldots, m\}\right\},
$$

where $C(\mathcal{S})$ is the set of continuous functions on $\mathcal{S}$.

For every $\phi \in H^{1}(\mathcal{S})$ denote

$$
\frac{d \phi}{d \mathbf{S}}(\mathbf{S}) \doteq \frac{d \phi}{d S_{1}}\left(\mathbf{A}^{\ell}+S_{1} \mathbf{t}_{1}^{\ell}\right) \quad \text { for a.e. } \mathbf{S}=\mathbf{A}^{\ell}+S_{1} \mathbf{t}_{1}^{\ell}, S_{1} \in\left(0, l_{\ell}\right), \ell \in\{1, \ldots, m\} .
$$

We endow $H^{1}(\mathcal{S})$ with the norm

$$
\|\psi\|_{H^{1}(\mathcal{S})} \doteq \sqrt{\|\psi\|_{L^{2}(\mathcal{S})}^{2}+\left\|\frac{d \psi}{d \mathbf{S}}\right\|_{L^{2}(\mathcal{S})}^{2}}, \quad \forall \psi \in H^{1}(\mathcal{S}) .
$$

\subsection{Stable Structures}

The space of all rigid displacements is denoted by $\mathbf{R}$

$$
\mathbf{R} \doteq\left\{\mathbf{r} \in C^{1}\left(\mathbb{R}^{3}\right) \mid \mathbf{r}(x)=\mathbf{a}+\mathbf{b} \wedge x, \quad \forall x \in \mathbb{R}^{3}, \quad(\mathbf{a}, \mathbf{b}) \in \mathbb{R}^{3} \times \mathbb{R}^{3}\right\} .
$$

We define the space $\mathbf{U}_{\mathcal{S}}$ as follows:

$\mathbf{U}_{\mathcal{S}} \doteq\left\{U \in C(\mathcal{S})^{3} \mid\right.$ for every segment $\gamma_{\ell} \subset \mathcal{S}, U_{\mid \gamma_{\ell}}$ is an affine function, $\left.\ell \in\{1, \ldots, m\}\right\}$.

Definition 2 A structure $\mathcal{S}$ is a stable structure if

$$
\forall U \in \mathbf{U}_{\mathcal{S}}, \quad \frac{d U}{d \mathbf{S}} \cdot \mathbf{t}_{1}=0 \quad \Longrightarrow \quad U \in \mathbf{R} .
$$

If the above condition is not satisfied, $\mathcal{S}$ is an unstable structure.

Remark 1 1. The structure made of the edges of a tetrahedron is stable (see Fig. 1.a). If we remove one edge then the structure becomes unstable (see Fig. 1.b).

2. The structure made of 12 edges and 6 diagonals of the faces of a cube is stable (see Fig. 1.c). If we remove one diagonal then the structure becomes unstable (see Fig. 1.d). 


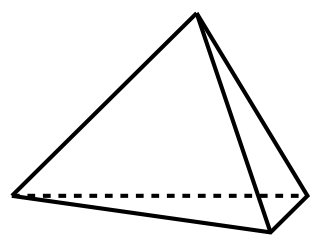

a. Stable tetrahedron

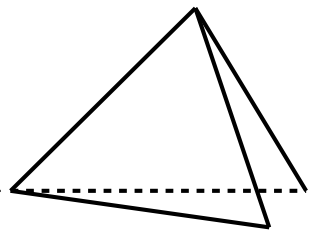

b. Unstable tetrahedron

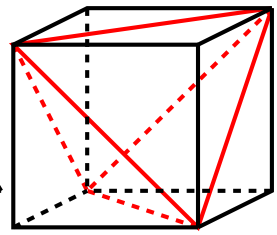

c. Stable cube

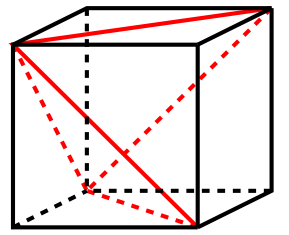

d. Unstable cube

Fig. 1 Stable and unstable structures

We equip $\mathbf{U}_{\mathcal{S}}$ with the following bilinear form:

$$
<\Phi, \Psi>_{1}=\int_{\mathcal{S}} \frac{\partial \Phi}{\partial S_{1}} \cdot \mathbf{t}_{1} \frac{\partial \Psi}{\partial S_{1}} \cdot \mathbf{t}_{1} d \mathbf{S}, \quad \forall(\Phi, \Psi) \in \mathbf{U}_{\mathcal{S}} \times \mathbf{U}_{\mathcal{S}}
$$

and the associated semi-norm

$$
\|U\|_{\mathcal{S}} \doteq \sqrt{<U, U>_{1}}=\left\|\frac{d U}{d \mathbf{S}} \cdot \mathbf{t}_{1}\right\|_{L^{2}(\mathcal{S})}, \quad \forall U \in \mathbf{U}_{\mathcal{S}} .
$$

Lemma 1 Let $\mathcal{S}$ be a stable structure. There exists a constant $C$, which depends on $\mathcal{S}$, such that for every $U$ in $\mathbf{U}_{\mathcal{S}}$ there exists $\mathbf{r} \in \mathbf{R}$ such that

$$
\|U-\mathbf{r}\|_{H^{1}(\mathcal{S})} \leq C\|U\|_{\mathcal{S}}
$$

Proof Let $\mathbf{R}^{\perp}$ be the orthonormal of $\mathbf{R}$ in $\mathbf{U}_{\mathcal{S}}$ for the scalar product

$$
<\Phi, \Psi>=\int_{\mathcal{S}} \Phi \cdot \Psi d \mathbf{S}, \quad \forall(\Phi, \Psi) \in \mathbf{U}_{\mathcal{S}} \times \mathbf{U}_{\mathcal{S}} .
$$

If $U$ belongs to $\mathbf{R}^{\perp}$ and satisfies $\|U\|_{\mathcal{S}}=0$ then, since $\mathcal{S}$ is a stable structure, $U$ belongs to R. Therefore $U$ is equal to 0 . The semi-norm $\|\cdot\|_{\mathcal{S}}$ is a norm on the space $\mathbf{R}^{\perp}$. Since $\mathbf{R}^{\perp}$ is a finite dimensional vector space, all the norms are equivalent. Thus (2.4) is proved.

\section{Decomposition of Beam Displacements}

In this section, we remind some results concerning the decomposition of a beam displacement. These results will be used later and can be found in [15]. For the sake of simplicity these results are formulated for the beam $B_{l, \mathfrak{r}} \doteq(0, l) \times D_{\mathfrak{r}}$ whose cross-sections are disc of radius $\mathfrak{r}(\mathfrak{r} \leq l)$. The beam is referred to the orthonormal frame $\left(O ; \mathbf{e}_{1}, \mathbf{e}_{2}, \mathbf{e}_{3}\right)\left(\mathbf{e}_{1}\right.$ is the direction of the centerline). In this frame the running point is denoted $x=\left(x_{1}, x_{2}, x_{3}\right)$.

Any displacement $u \in H^{1}\left(B_{l, \mathfrak{r}}\right)^{3}$ of the beam $B_{l, \mathfrak{r}}$ is uniquely decomposed as follows

$$
u=U^{e}+\bar{u}
$$

where $U^{e}$ is called elementary displacement and it stands for the displacement of the centerline of the beam and the small rotation of the cross-section at every point of the centerline (see Fig. 2):

$$
U^{e}(x)=\mathcal{U}\left(x_{1}\right)+\mathcal{R}\left(x_{1}\right) \wedge\left(x_{2} \mathbf{e}_{2}+x_{3} \mathbf{e}_{3}\right), \quad \text { for a.e. } x=\left(x_{1}, x_{2}, x_{3}\right) \in B_{l, \mathfrak{r}} .
$$


Fig. 2 Beam $B_{\ell, \mathfrak{r}}$

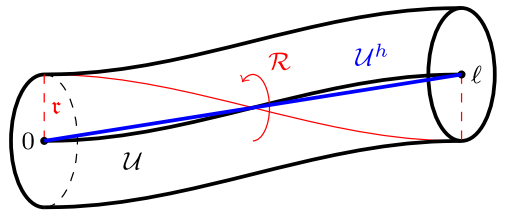

$\mathcal{U}=\left(\mathcal{U}_{1}, \mathcal{U}_{2}, \mathcal{U}_{3}\right)$ and $\mathcal{R}=\left(\mathcal{R}_{1}, \mathcal{R}_{2}, \mathcal{R}_{3}\right)$ belong to $H^{1}(0, l)^{3}$. The residual displacement $\bar{u} \in$ $H^{1}\left(B_{l, \mathfrak{r}}\right)^{3}$ is the warping (the deformation of the cross-sections), it satisfies (for more details see [15])

$$
\int_{D_{\mathfrak{r}}} \bar{u}(x) d x_{2} d x_{3}=\int_{D_{\mathfrak{r}}} \bar{u}(x) \wedge\left(x_{2} \mathbf{e}_{2}+x_{3} \mathbf{e}_{3}\right) d x_{2} d x_{3}=0 \quad \text { for a.e. } x_{1} \in(0, l) .
$$

Taking into account the decomposition (3.1) and the representation for the elementary displacement given by (3.2) the strain tensor $e(u)$ has the following form:

$$
\begin{aligned}
& e(u)=e\left(U^{e}\right)+e(\bar{u}) \\
& =\left(\begin{array}{ccc}
\frac{d \mathcal{U}_{1}}{d x_{1}}-x_{2} \frac{d \mathcal{R}_{3}}{d x_{1}}+x_{3} \frac{d \mathcal{R}_{2}}{d x_{1}} & \frac{1}{2}\left[\left(\frac{d \mathcal{U}_{2}}{d x_{1}}-\mathcal{R}_{3}\right)-x_{3} \frac{d \mathcal{R}_{1}}{d x_{1}}\right] & \frac{1}{2}\left[\left(\frac{d \mathcal{U}_{3}}{d x_{1}}+\mathcal{R}_{2}\right)+x_{2} \frac{d \mathcal{R}_{1}}{d x_{1}}\right] \\
\frac{1}{2}\left[\left(\frac{d \mathcal{U}_{2}}{d x_{1}}-\mathcal{R}_{3}\right)-x_{3} \frac{d \mathcal{R}_{1}}{d x_{1}}\right] & 0 & 0 \\
\frac{1}{2}\left[\left(\frac{d \mathcal{U}_{3}}{d x_{1}}+\mathcal{R}_{2}\right)+x_{2} \frac{d \mathcal{R}_{1}}{d x_{1}}\right] & 0 & 0
\end{array}\right) \\
& +e(\bar{u}) \text {. }
\end{aligned}
$$

Below is a lemma proven in $[13,15]$. It gives estimates for the warping and the terms from $U^{e}$ in the above strain tensor (3.4).

Lemma 2 Let $u$ be in $H^{1}\left(B_{l, \mathfrak{r}}\right)^{3}$ decomposed as (3.1)-(3.2)-(3.3). The following estimates hold:

$$
\begin{aligned}
& \|\bar{u}\|_{L^{2}\left(B_{l, \mathfrak{r}}\right)} \leq C \mathfrak{r}\|e(u)\|_{L^{2}\left(B_{l, \mathfrak{r}}\right)}, \quad\|\nabla \bar{u}\|_{L^{2}\left(B_{l, \mathfrak{r}}\right)} \leq C\|e(u)\|_{L^{2}\left(B_{l, \mathfrak{r}}\right)}, \\
& \left\|\frac{d \mathcal{R}}{d x_{1}}\right\|_{L^{2}(0, l)} \leq \frac{C}{\mathfrak{r}^{2}}\|e(u)\|_{L^{2}\left(B_{l, \mathfrak{r}}\right)}, \quad\left\|\frac{d \mathcal{U}}{d x_{1}}-\mathcal{R} \wedge \mathbf{e}_{1}\right\|_{L^{2}(0, l)} \leq \frac{C}{\mathfrak{r}}\|e(u)\|_{L^{2}\left(B_{l, \mathfrak{r}}\right)} .
\end{aligned}
$$

The constants are independent of $l$ and $\mathfrak{r} \leq l$.

The function $\mathcal{U}$, defined in (3.1), is decomposed into the sum of two functions $\mathcal{U}^{h}$ and $\overline{\mathcal{U}}$, where $\mathcal{U}^{h}$ coincides with $\mathcal{U}$ in the extremities of the centerline and is laffine between them (see Fig. 2), and $\overline{\mathcal{U}}=\mathcal{U}-\mathcal{U}^{h}$ is the residual part, i.e.,

$$
\mathcal{U}^{h}\left(x_{1}\right)=\frac{\left(l-x_{1}\right)}{l} \mathcal{U}(0)+\frac{x_{1}}{l} \mathcal{U}(l)
$$

In the same way the function $\mathcal{R}$, defined in (3.1), is decomposed into the sum of two functions $\mathcal{R}^{h}$ and $\overline{\mathcal{R}}$. It is obvious, but important to note that

$$
\overline{\mathcal{U}}(0)=\overline{\mathcal{U}}(l)=0, \quad \overline{\mathcal{R}}(0)=\overline{\mathcal{R}}(l)=0 .
$$


Lemma 3 The following estimates hold:

$$
\begin{aligned}
& \left\|\frac{d \overline{\mathcal{R}}}{d x_{1}}\right\|_{L^{2}(0, l)} \leq \frac{C}{\mathfrak{r}^{2}}\|e(u)\|_{L^{2}\left(B_{l, \mathfrak{r}}\right)}, \quad\|\overline{\mathcal{R}}\|_{L^{2}(0, l)} \leq \frac{C l}{\mathfrak{r}^{2}}\|e(u)\|_{L^{2}\left(B_{l, \mathfrak{r}}\right)} \\
& \left\|\frac{d \overline{\mathcal{U}}}{d x_{1}} \cdot \mathbf{e}_{1}\right\|_{L^{2}(0, l)} \leq \frac{C}{\mathfrak{r}}\|e(u)\|_{L^{2}\left(B_{l, \mathfrak{r}}\right)}, \quad\left\|\frac{d \overline{\mathcal{U}}}{d x_{1}}\right\|_{L^{2}(0, l)} \leq C \frac{l}{\mathfrak{r}^{2}}\|e(u)\|_{L^{2}\left(B_{l, \mathfrak{r}}\right)}, \\
& \left\|\overline{\mathcal{U}} \cdot \mathbf{e}_{1}\right\|_{L^{2}(0, l)} \leq C \frac{l}{\mathfrak{r}}\|e(u)\|_{L^{2}\left(B_{l, \mathfrak{r}}\right)}, \quad\|\overline{\mathcal{U}}\|_{L^{2}(0, l)} \leq C \frac{l^{2}}{\mathfrak{r}^{2}}\|e(u)\|_{L^{2}\left(B_{l, \mathfrak{r}}\right)}, \\
& \left\|\frac{d \mathcal{U}^{h}}{d x_{1}}-\mathcal{R}^{h} \wedge \mathbf{e}_{1}\right\|_{L^{2}(0, l)} \leq \frac{C l}{\mathfrak{r}^{2}}\|e(u)\|_{L^{2}\left(B_{l, \mathfrak{r}}\right)}, \\
& \left\|\frac{d \mathcal{R}^{h}}{d x_{1}}\right\|_{L^{2}(0, l)}+\frac{1}{\mathfrak{r}}\left\|\frac{d \mathcal{U}^{h}}{d x_{1}} \cdot \mathbf{e}_{1}\right\|_{L^{2}(0, l)} \leq \frac{C}{\mathfrak{r}^{2}}\|e(u)\|_{L^{2}\left(B_{l, \mathfrak{r}}\right)} .
\end{aligned}
$$

The constants do not depend on $l$ and $\mathfrak{r}$.

Proof Since $\frac{d \mathcal{R}^{h}}{d x_{1}}$ and $\frac{d \overline{\mathcal{U}}}{d x_{1}}-(\mathcal{R}-m(\mathcal{R})) \wedge \mathbf{e}_{1}\left(m(\mathcal{R})=\frac{1}{l} \int_{0}^{l} \mathcal{R}(t) d t\right)$ are constant on $(0, l)$, one gets

$$
\begin{aligned}
& \left\|\frac{d \mathcal{R}^{h}}{d x_{1}}\right\|_{L^{2}(0, l)}^{2}+\left\|\frac{d \overline{\mathcal{R}}}{d x_{1}}\right\|_{L^{2}(0, l)}^{2}=\left\|\frac{d \mathcal{R}}{d x_{1}}\right\|_{L^{2}(0, l)}^{2} \leq \frac{C}{\mathfrak{r}^{4}}\|e(u)\|_{L^{2}\left(B_{l, \mathfrak{r}}\right)}^{2}, \\
& \left\|\frac{d \mathcal{U}^{h}}{d x_{1}}-m(\mathcal{R}) \wedge \mathbf{e}_{1}\right\|_{L^{2}(0, l)}^{2}+\left\|\frac{d \overline{\mathcal{U}}}{d x_{1}}-(\mathcal{R}-m(\mathcal{R})) \wedge \mathbf{e}_{1}\right\|_{L^{2}(0, l)}^{2}=\left\|\frac{d \mathcal{U}}{d x_{1}}-\mathcal{R} \wedge \mathbf{e}_{1}\right\|_{L^{2}(0, l)}^{2} \\
& \quad \leq \frac{C}{\mathfrak{r}^{2}}\|e(u)\|_{L^{2}\left(B_{l, \mathfrak{r}}\right)}^{2} .
\end{aligned}
$$

Then, the Poincaré and the Poincaré-Wirtinger inequalities together with the above estimates yield

$$
\begin{aligned}
& \left\|\mathcal{R}-\mathcal{R}^{h}\right\|_{L^{2}(0, l)}=\|\overline{\mathcal{R}}\|_{L^{2}(0, l)} \leq C \frac{l}{\mathfrak{r}^{2}}\|e(u)\|_{L^{2}\left(B_{l, \mathfrak{r}}\right)} \quad \text { and } \\
& \|\mathcal{R}-m(\mathcal{R})\|_{L^{2}(0, l)} \leq C \frac{l}{\mathfrak{r}^{2}}\|e(u)\|_{L^{2}\left(B_{l, \mathfrak{r}}\right)},
\end{aligned}
$$

from which we derive the other estimates in (3.6).

\section{Decomposition of the Displacements of a Beam Structure}

From now on, $\mathcal{S}$ is a stable structure.

The beam structure $\mathcal{S}_{1, \mathfrak{r}}$ is defined as follows:

$$
\mathcal{S}_{1, \mathfrak{r}}=\left\{x \in \mathbb{R}^{3} \mid \operatorname{dist}(x, \mathcal{S})<\mathfrak{r}\right\} .
$$

For $\ell \in\{1, \ldots, m\}$, denote $\mathcal{P}_{\ell, \mathfrak{r}}$ the straight beam with centerline $\gamma_{\ell}=\left[\mathbf{A}^{\ell}, \mathbf{B}^{\ell}\right]$ and reference cross-section the disk $D_{\mathfrak{r}} \doteq D(O, \mathfrak{r})$ of radius $\mathfrak{r}, 0<\mathfrak{r} \leq l_{\ell}$ (the disk $D_{1}$ for simplicity will be denoted $D$ ). The straight beam $\mathcal{P}_{\ell, \mathfrak{r}}$ is referred to the orthonormal frame $\left(\mathbf{A}^{\ell} ; \mathbf{t}_{1}^{\ell}, \mathbf{t}_{2}^{\ell}, \mathbf{t}_{3}^{\ell}\right)$

$$
\mathcal{P}_{\ell, \mathfrak{r}}=\left\{x \in \mathbb{R}^{3} \mid x=\mathbf{A}^{\ell}+S_{1} \mathbf{t}_{1}^{\ell}+S_{2} \mathbf{t}_{2}^{\ell}+S_{3} \mathbf{t}_{3}^{\ell}, \quad\left(S_{1}, S_{2}, S_{3}\right) \in\left(0, l_{\ell}\right) \times D_{\mathfrak{r}}\right\} .
$$


By definition, the whole structure $\mathcal{S}_{1, \mathfrak{r}}$ contains the straight beams $\mathcal{P}_{\ell, \mathfrak{r}}, \ell \in\{1, \ldots, m\}$ and the balls of radius $\mathfrak{r}$ centered in the points of $\mathcal{K}$, more precisely one has

$$
\mathcal{S}_{1, \mathfrak{r}} \doteq\left(\bigcup_{A \in \mathcal{K}} B(A, \mathfrak{r})\right) \cup\left(\bigcup_{\ell=1}^{m} \mathcal{P}_{\ell, \mathfrak{r}}\right) \text {. }
$$

The set of junction domains is denoted by $\mathcal{J}_{\mathfrak{r}}$. There exists $c_{0}$ which only depends on $\mathcal{S}$ such that

$$
\mathcal{J}_{\mathfrak{r}} \subset \bigcup_{A \in \mathcal{K}} B\left(A, c_{0} \mathfrak{r}\right)
$$

The set $\mathcal{J}_{\mathfrak{r}}$ is defined in such a way that $\mathcal{S}_{1, \mathfrak{r}} \backslash \overline{\mathcal{J}}_{\mathfrak{r}}$ only consists of disjoint straight beams.

Definition 3 An elementary beam-structure displacement is a displacement $U^{e}$ belonging to $H^{1}\left(\mathcal{S}_{1, \mathfrak{r}}\right)^{3}$ whose restriction to each beam is an elementary displacement and whose restriction to each junction is a rigid displacement

$$
\begin{aligned}
U^{e}(x)= & \mathcal{U}\left(\mathbf{A}^{\ell}+S_{1} \mathbf{t}_{1}^{\ell}\right)+\mathcal{R}\left(\mathbf{A}^{\ell}+S_{1} \mathbf{t}_{1}^{\ell}\right) \wedge\left(S_{2} \mathbf{t}_{2}^{\ell}+S_{3} \mathbf{t}_{3}^{\ell}\right), \\
\text { for a.e. } x=\mathbf{A}^{\ell}+S_{1} \mathbf{t}_{1}^{\ell}+S_{2} \mathbf{t}_{2}^{\ell}+S_{3} \mathbf{t}_{3}^{\ell} \in \mathcal{P}_{\ell, \mathfrak{r}}, & \\
& \left(S_{1}, S_{2}, S_{3}\right) \in\left(0, l_{\ell}\right) \times D_{\mathfrak{r}}, \ell \in\{1, \ldots, m\}, \\
U^{e}(x)= & \mathcal{U}(A)+\mathcal{R}(A) \wedge(x-A), \quad \text { for a.e. } x \in B(A, r), \quad \text { for all } A \in \mathcal{K}
\end{aligned}
$$

with $\mathcal{U}$ and $\mathcal{R}$ in $H^{1}(\mathcal{S})^{3}$.

In [14] it is shown that every displacement $u \in H^{1}\left(\mathcal{S}_{1, \mathfrak{r}}\right)^{3}$ can be decomposed as

$$
u=U^{e}+\bar{u}
$$

where $U^{e}$ is an elementary beam-structure displacement and where $\bar{u} \in H^{1}\left(\mathcal{S}_{1, \mathfrak{r}}\right)^{3}$ is the warping. Here, the pair $\left(U^{e}, \bar{u}\right)$ is not uniquely determined. Furthermore, the warping satisfies the conditions (3.3) "outside" the domain $\mathcal{J}_{\mathfrak{r}}$ (see $[14,15]$ ), more precisely, one has

$$
\begin{aligned}
& \int_{D_{\mathfrak{r}}} \bar{u}\left(\cdot, S_{2}, S_{3}\right) d S_{2} d S_{3}=0 \\
& \int_{D_{\mathfrak{r}}} \bar{u}\left(\cdot, S_{2}, S_{3}\right) \wedge\left(S_{2} \mathbf{e}_{2}+S_{3} \mathbf{e}_{3}\right) d S_{2} d S_{3}=0,
\end{aligned}
$$

The following lemma is proved in [14, Lemma 3.4]:

Lemma 4 Let $u$ be in $H^{1}\left(\mathcal{S}_{1, \mathfrak{r}}\right)^{3}$. There exists a decomposition of $u, u=U^{e}+\bar{u}$ for which $U^{e}$ is an elementary beam-structure displacement. The terms of this decomposition satisfy

$$
\begin{aligned}
& \|\bar{u}\|_{L^{2}\left(\mathcal{S}_{1, \mathfrak{r}}\right)} \leq C \mathfrak{r}\|e(u)\|_{L^{2}\left(\mathcal{S}_{1, \mathfrak{r}}\right)}, \quad\|\nabla \bar{u}\|_{L^{2}\left(\mathcal{S}_{1, \mathfrak{r}}\right)} \leq C\|e(u)\|_{L^{2}\left(\mathcal{S}_{1, \mathfrak{r}}\right)}, \\
& \left\|\frac{d \mathcal{R}}{d \mathbf{S}}\right\|_{L^{2}(\mathcal{S})} \leq \frac{C}{\mathfrak{r}^{2}}\|e(u)\|_{L^{2}\left(\mathcal{S}_{1, \mathfrak{r}}\right)}, \quad\left\|\frac{d \mathcal{U}}{d \mathbf{S}}-\mathcal{R} \wedge \mathbf{t}_{1}\right\|_{L^{2}(\mathcal{S})} \leq \frac{C}{\mathfrak{r}}\|e(u)\|_{L^{2}\left(\mathcal{S}_{1, \mathfrak{r}}\right)} .
\end{aligned}
$$

The constants do not depend on $\mathfrak{r}$. 
Here, again we split the field $\mathcal{U}$ into the sum of two fields $\mathcal{U}^{h}$ and $\overline{\mathcal{U}}$, where $\mathcal{U}^{h}$ coincides with $\mathcal{U}$ in the nodes of $\mathcal{S}$ and is affine between two contiguous nodes and $\overline{\mathcal{U}}=\mathcal{U}-\mathcal{U}^{h}$ is the residual part.

In the same way the fields $\mathcal{R}^{h}$ and $\overline{\mathcal{R}}$ are introduced. The field $\mathcal{U}^{h}$ describes the displacement of the nodes, i.e. the global behavior of the structure, whereas $\overline{\mathcal{U}}$ stands for the local displacement of the beams.

By construction the fields $\mathcal{U}^{h}$ and $\mathcal{R}^{h}$ belong to $\mathbf{U}_{\mathcal{S}}$. Furthermore one has

Lemma 5 For every $u \in H^{1}\left(\mathcal{S}_{1, \mathfrak{r}}\right)^{3}$ the following estimates hold:

$$
\begin{aligned}
& \left\|\frac{d \overline{\mathcal{R}}}{d \mathbf{S}}\right\|_{L^{2}(\mathcal{S})}+\|\overline{\mathcal{R}}\|_{L^{2}(\mathcal{S})} \leq \frac{C}{\mathfrak{r}^{2}}\|e(u)\|_{L^{2}\left(\mathcal{S}_{1, \mathfrak{r}}\right)}, \\
& \left\|\frac{d \overline{\mathcal{U}}}{d \mathbf{S}} \cdot \mathbf{t}_{1}\right\|_{L^{2}(\mathcal{S})}+\left\|\overline{\mathcal{U}} \cdot \mathbf{t}_{1}\right\|_{L^{2}(\mathcal{S})} \leq \frac{C}{\mathfrak{r}}\|e(u)\|_{L^{2}\left(\mathcal{S}_{1, \mathfrak{r}}\right)}, \\
& \left\|\frac{d \overline{\mathcal{U}}}{d \mathbf{S}}\right\|_{L^{2}(\mathcal{S})}+\|\overline{\mathcal{U}}\|_{L^{2}(\mathcal{S})} \leq \frac{C}{\mathfrak{r}^{2}}\|e(u)\|_{L^{2}\left(\mathcal{S}_{1, \mathfrak{r}}\right)}, \\
& \left\|\frac{d \mathcal{U}^{h}}{d \mathbf{S}}-\mathcal{R}^{h} \wedge \mathbf{t}_{1}\right\|_{L^{2}(\mathcal{S})}+\left\|\frac{d \mathcal{R}^{h}}{d \mathbf{S}}\right\|_{L^{2}(\mathcal{S})}+\frac{1}{\mathfrak{r}}\left\|\frac{d \mathcal{U}^{h}}{d \mathbf{S}} \cdot \mathbf{t}_{1}\right\|_{L^{2}(\mathcal{S})} \leq \frac{C}{\mathfrak{r}^{2}}\|e(u)\|_{L^{2}\left(\mathcal{S}_{1, \mathfrak{r}}\right)} .
\end{aligned}
$$

Moreover, since $\mathcal{S}$ is a stable structure, there exists a rigid displacement $\mathbf{r} \in \mathbf{R},(\mathbf{r}(x)=$ $\mathbf{a}+\mathbf{b} \wedge x)$, such that

$$
\left\|\mathcal{U}^{h}-\mathbf{r}\right\|_{H^{1}(\mathcal{S})} \leq \frac{C}{\mathfrak{r}}\|e(u)\|_{L^{2}\left(\mathcal{S}_{1, \mathfrak{r}}\right)}, \quad\left\|\mathcal{R}^{h}-\mathbf{b}\right\|_{L^{2}(\mathcal{S})} \leq \frac{C}{\mathfrak{r}^{2}}\|e(u)\|_{L^{2}\left(\mathcal{S}_{1, \mathfrak{r}}\right)} .
$$

The constants do not depend on $\mathfrak{r}$.

Proof Estimates (4.4) are the immediate consequences of the Lemmas 3 and 4. Since $\mathcal{S}$ is a stable structure, Lemma 1 and again (4.4) yield a rigid displacement $\mathbf{r} \in \mathbf{R}(\mathbf{r}(x)=\mathbf{a}+\mathbf{b} \wedge x)$ such that $(4.5)_{1}$ holds.

Besides, from the Poincaré-Wirtinger inequality and (4.4) $)_{4}$, there exists $\widetilde{\mathbf{b}} \in \mathbb{R}^{3}$ such that

$$
\left\|\mathcal{R}^{h}-\widetilde{\mathbf{b}}\right\|_{L^{2}(\mathcal{S})} \leq \frac{C}{\mathfrak{r}^{2}}\|e(u)\|_{L^{2}\left(\mathcal{S}_{1, \mathfrak{r}}\right)} .
$$

The constant does not depend on $\mathfrak{r}$. Then, $(4.5)_{1}$ and the above estimate give

$$
\left\|(\mathbf{b}-\widetilde{\mathbf{b}}) \wedge \mathbf{t}_{1}\right\|_{L^{2}(\mathcal{S})} \leq \frac{C}{\mathfrak{r}^{2}}\|e(u)\|_{L^{2}\left(\mathcal{S}_{1, \mathfrak{r}}\right)} .
$$

Since the structure has more than two segments with non-collinear directions, this yields

$$
|\mathbf{b}-\widetilde{\mathbf{b}}| \leq \frac{C}{\mathfrak{r}^{2}}\|e(u)\|_{L^{2}\left(\mathcal{S}_{1, \mathfrak{r}}\right)} .
$$

Hence, $(4.5)_{2}$ is proved.

Let $\mathcal{S}$ be a stable structure such that $\mathcal{S} \cup\left(\mathcal{S}+\mathbf{e}_{1}\right)$ is a stable structure. For every displacement $u \in H^{1}\left(\mathcal{S}_{1, \mathfrak{r}} \cup\left(\mathcal{S}_{1, \mathfrak{r}}+\mathbf{e}_{1}\right)\right)^{3}$, Lemma 5 gives two rigid displacements $\mathbf{r}_{0}, \mathbf{r}_{1}$ such 
that

$$
\begin{aligned}
& \mathbf{r}_{0}(x)=\mathbf{a}_{0}+\mathbf{b}_{0} \wedge(x-G), \quad \mathbf{r}_{1}(x)=\mathbf{a}_{1}+\mathbf{b}_{1} \wedge\left(x-G-\mathbf{e}_{1}\right) \quad \forall x \in \mathbb{R}^{3}, \\
& \left\|\mathcal{U}^{h}-\mathbf{r}_{0}\right\|_{H^{1}(\mathcal{S})} \quad \leq \frac{C}{\mathfrak{r}}\|e(u)\|_{L^{2}\left(\mathcal{S}_{1, \mathfrak{r}}\right)}, \quad\left\|\mathcal{U}^{h}-\mathbf{r}_{1}\right\|_{H^{1}\left(\mathcal{S}+\mathbf{e}_{1}\right)} \leq \frac{C}{\mathfrak{r}}\|e(u)\|_{L^{2}\left(\mathcal{S}+\mathbf{e}_{1}\right)},
\end{aligned}
$$

where $G$ is the center of mass of $\mathcal{S}$.

Lemma 6 Let $\mathcal{S}$ be a stable structure such that $\mathcal{S} \cup\left(\mathcal{S}+\mathbf{e}_{1}\right)$ is also a stable structure. The following estimate holds:

$$
\left\|\mathbf{r}_{1}-\mathbf{r}_{0}\right\|_{H^{1}\left(\mathcal{S} \cup\left(\mathcal{S}+\mathbf{e}_{1}\right)\right)} \leq \frac{C}{\mathfrak{r}}\|e(u)\|_{L^{2}\left(\mathcal{S}_{1, \mathfrak{r}} \cup\left(\mathcal{S}_{1, \mathfrak{r}}+\mathbf{e}_{1}\right)\right)} .
$$

The constant does not depend on $\mathfrak{r}$.

Proof From Lemma 5, there exists a rigid displacement $\mathbf{r}$ such that

$$
\begin{aligned}
& \mathbf{r}(x)=\mathbf{a}+\mathbf{b} \wedge\left(x-G-\mathbf{e}_{1} / 2\right) \quad \forall x \in \mathbb{R}^{3}, \\
& \left\|\mathcal{U}^{h}-\mathbf{r}\right\|_{H^{1}\left(\mathcal{S} \cup\left(\mathcal{S}+\mathbf{e}_{1}\right)\right)} \leq \frac{C}{\mathfrak{r}}\|e(u)\|_{L^{2}\left(\mathcal{S}_{1, \mathfrak{r}} \cup\left(\mathcal{S}_{1, \mathfrak{r}}+\mathbf{e}_{1}\right)\right)} .
\end{aligned}
$$

The constant does not depend on $\mathfrak{r}$. Hence

$$
\begin{aligned}
& \left\|\mathbf{r}-\mathbf{r}_{0}\right\|_{H^{1}(\mathcal{S})} \leq \frac{C}{\mathfrak{r}}\|e(u)\|_{L^{2}\left(\mathcal{S}_{1, \mathfrak{r}} \cup\left(\mathcal{S}_{1, \mathfrak{r}}+\mathbf{e}_{1}\right)\right)}, \\
& \left\|\mathbf{r}-\mathbf{r}_{1}\right\|_{H^{1}\left(\mathcal{S}+\mathbf{e}_{1}\right)} \leq \frac{C}{\mathfrak{r}}\|e(u)\|_{L^{2}\left(\mathcal{S}_{1, \mathfrak{r}} \cup\left(\mathcal{S}_{1, \mathfrak{r}}+\mathbf{e}_{1}\right)\right)} .
\end{aligned}
$$

The above estimates yield (4.7) since in $\mathbf{R}$ the norms $\|\cdot\|_{H^{1}(\mathcal{S})},\|\cdot\|_{H^{1}\left(\mathcal{S}+\mathbf{e}_{1}\right)}$ and $\| \cdot$ $\|_{H^{1}\left(\mathcal{S} \cup\left(\mathcal{S}+\mathbf{e}_{1}\right)\right)}$ are equivalent.

\section{A Periodic Beam Structure as 3D-Like Domain}

From now on, in all the estimates, we denote by $C$ a strictly positive constant which does not depend on $\varepsilon$ and $r$.

\subsection{Notations and Statement of the Problem}

Below we consider periodic structures $\mathcal{S}$ included in a closed parallelotope.

Definition 4 A structure $\mathcal{S}$ is a $3 D$-periodic structure if for every $i \in\{1,2,3\}$ the set $\mathcal{S} \cup\left(\mathcal{S}+\mathbf{e}_{i}\right)$ is a structure in the sense of Definition 1.

Definition 5 A $3 D$-periodic structure $\mathcal{S}$ is a $3 D$-periodic stable structure (briefly 3-PSS) if $\mathcal{S}$ and $\mathcal{S} \cup\left(\mathcal{S}+\mathbf{e}_{i}\right), i \in\{1,2,3\}$, are stable structures in the sense of Definition 2.

Remark 2 1. The structure made of 12 edges and 6 diagonals of the faces of a cube is a $3 D$-periodic stable structure (Fig. 3.a). 

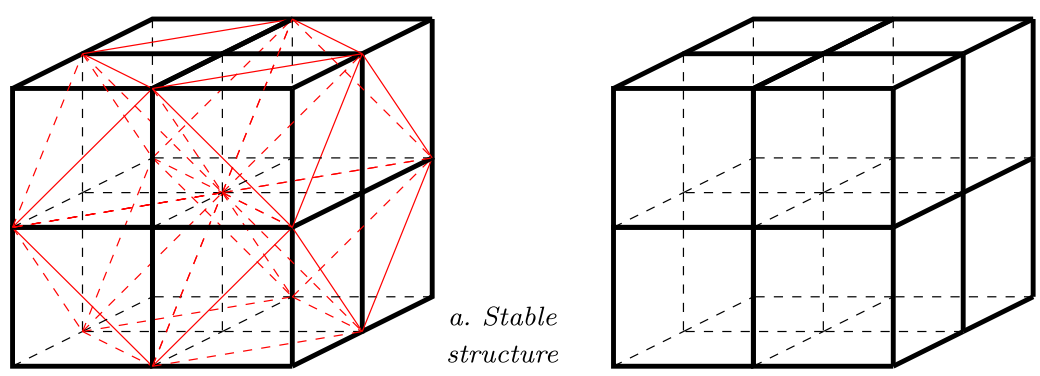

b. Unstable

a. Stable structure structure

Fig. 3 3D-periodic stable and unstable structures

2. The structure made of 12 edges of a cube is not a $3 D$-periodic stable structure (Fig. 3.b).

Let $\Omega$ be a bounded domain in $\mathbb{R}^{3}$ with a Lipschitz boundary and $\Gamma$ be a subset of $\partial \Omega$ with nonnull measure. We assume that there exists an open set $\Omega^{\prime}$ with a Lipschitz boundary such that $\Omega \subset \Omega^{\prime}$ and $\Omega^{\prime} \cap \partial \Omega=\Gamma$.

Denote

$-\Omega_{1} \doteq\left\{x \in \mathbb{R}^{N} \mid \operatorname{dist}(x, \Omega)<1\right\}, \Omega_{\varepsilon}^{i n t}=\{x \in \Omega \mid \operatorname{dist}(x, \partial \Omega)>2 \sqrt{3} \varepsilon\}$,

$-Y \doteq(0,1)^{3}$,

- $G=(1 / 2,1 / 2,1 / 2)$ the center of mass of $Y$,

- $\mathcal{S}$ a 3-periodic structure included in $\bar{Y}$,

$-\boldsymbol{\Xi}_{\varepsilon} \doteq\left\{\xi \in \mathbb{Z}^{3} \mid(\varepsilon \xi+\varepsilon Y) \cap \Omega \neq \emptyset\right\}, \widetilde{\Xi}_{\varepsilon} \doteq\left\{\xi \in \mathbb{Z}^{3} \mid(\varepsilon \xi+\varepsilon Y) \subset \Omega\right\}$

- $\Xi_{\varepsilon}^{i n t} \doteq\left\{\xi \in \mathbb{Z}^{3} \mid(\varepsilon \xi+\varepsilon Y) \subset \Omega_{\varepsilon}^{i n t}\right\}$,

- $\Xi_{\varepsilon}^{\prime} \doteq\left\{\xi \in \mathbb{Z}^{3} \mid(\varepsilon \xi+\varepsilon Y) \cap \Omega^{\prime} \neq \emptyset\right\}$,

- $\widehat{\Xi}_{\varepsilon} \doteq\left\{\xi \in \Xi_{\varepsilon} \mid\right.$ all the vertices of $\xi+\bar{Y}$ belong to $\left.\Xi_{\varepsilon}\right\}$,

- $\boldsymbol{\Xi}_{\varepsilon, i} \doteq\left\{\xi \in \mathbf{\Xi}_{\varepsilon} \mid \xi+\mathbf{e}_{i} \in \mathbf{\Xi}_{\varepsilon}\right\}, i \in\{1,2,3\}$,

$-\Omega_{\varepsilon} \doteq \operatorname{interior}\left(\bigcup_{\xi \in \Xi_{\varepsilon}}(\varepsilon \xi+\varepsilon \bar{Y})\right), \quad \widehat{\Omega}_{\varepsilon} \doteq \operatorname{interior}\left(\bigcup_{\xi \in \widehat{\Xi}_{\varepsilon}}(\varepsilon \xi+\varepsilon \bar{Y})\right), \quad \Omega_{\varepsilon}^{\prime} \doteq$ interior $\left(\bigcup_{\xi \in \Xi_{\varepsilon}^{\prime}}(\varepsilon \xi+\varepsilon \bar{Y})\right)$

$-\widehat{\Omega}_{\varepsilon}^{i n t} \doteq \operatorname{interior}\left(\bigcup_{\xi \in \Xi_{\varepsilon}^{i n t}}(\varepsilon \xi+\varepsilon \bar{Y})\right), \widetilde{\Omega}_{\varepsilon} \doteq \operatorname{interior}\left(\bigcup_{\xi \in \widetilde{\Xi}_{\varepsilon}}(\varepsilon \xi+\varepsilon \bar{Y})\right)$.

One has

$$
\Xi_{\varepsilon}^{i n t} \subset \widehat{\Xi}_{\varepsilon} \subset \bigcap_{i=1}^{3} \Xi_{\varepsilon, i} \subset \bigcup_{i=1}^{3} \Xi_{\varepsilon, i}=\Xi_{\varepsilon} .
$$

The open sets $\Omega_{\varepsilon}, \Omega_{\varepsilon}^{\prime}, \widehat{\Omega}_{\varepsilon}, \widehat{\Omega}_{\varepsilon}^{i n t}$ and $\Omega_{\varepsilon}^{i n t}$ are connected. Moreover, the following inclusions hold

$$
\widehat{\Omega}_{\varepsilon}^{i n t} \subset \Omega_{\varepsilon}^{i n t} \subset \Omega \subset \Omega_{\varepsilon} \subset \Omega_{\varepsilon}^{\prime}, \quad \widehat{\Omega}_{\varepsilon}^{i n t} \subset \Omega_{\varepsilon}^{i n t} \subset \widehat{\Omega}_{\varepsilon} \subset \Omega_{\varepsilon}
$$


Set

$$
\begin{aligned}
& \mathcal{S}_{\varepsilon} \doteq \bigcup_{\xi \in \Xi_{\varepsilon}}(\varepsilon \xi+\varepsilon \mathcal{S}), \quad \mathcal{S}_{\varepsilon, r} \doteq\left\{x \in \mathbb{R}^{3} \mid \operatorname{dist}\left(x, \mathcal{S}_{\varepsilon}\right)<r\right\}, \\
& \mathcal{S}_{\varepsilon}^{\prime} \doteq \bigcup_{\xi \in \Xi_{\varepsilon}^{\prime}}(\varepsilon \xi+\varepsilon \mathcal{S}), \quad \mathcal{S}_{\varepsilon, r}^{\prime} \doteq\left\{x \in \mathbb{R}^{3} \mid \operatorname{dist}\left(x, \mathcal{S}_{\varepsilon}^{\prime}\right)<r\right\}, \\
& \mathcal{K}_{\varepsilon} \doteq \bigcup_{\xi \in \Xi_{\varepsilon}}(\varepsilon \xi+\varepsilon \mathcal{K}) .
\end{aligned}
$$

The running point of $\mathcal{S}_{\varepsilon}$ is denoted $\mathbf{s}$.

Let $\mathcal{S}_{\varepsilon, r}$ be a beam structure consisting of balls of radius $r$ centered on the points of $\mathcal{K}_{\varepsilon}$ and beams, whose cross-sections are discs of radius $r$ and their centerlines are the segments of $\mathcal{S}_{\varepsilon}$

$$
\begin{aligned}
& \mathcal{P}_{\varepsilon \ell, r}^{\xi} \doteq \varepsilon \xi+\varepsilon \mathcal{P}_{\ell, \mathfrak{r}}, \quad \ell \in\{1, \ldots, m\}, \quad \mathfrak{r}=r / \varepsilon, \\
& \mathcal{S}_{\varepsilon, r} \doteq\left(\bigcup_{A \in \mathcal{K}_{\varepsilon}} B(A, r)\right) \cup\left(\bigcup_{\xi \in \Xi_{\varepsilon}} \bigcup_{\ell=1}^{m} \mathcal{P}_{\varepsilon \ell, r}^{\xi}\right) .
\end{aligned}
$$

The parametrization of the beam $\mathcal{P}_{\varepsilon \ell, r}^{\xi}(\ell \in\{1, \ldots, m\})$ is given by (see (4.1))

$$
x=\varepsilon \xi+\varepsilon \mathbf{A}^{\ell}+s_{1} \mathbf{t}_{1}^{\ell}+s_{2} \mathbf{t}_{2}^{\ell}+s_{3} \mathbf{t}_{3}^{\ell}, \quad\left(s_{1}, s_{2}, s_{3}\right) \in\left(0, \varepsilon l_{\ell}\right) \times D_{r} .
$$

The junction domains (the common parts of the beams) is denoted $\mathcal{J}_{\varepsilon, r}$. One has

$$
\bigcup_{A \in \mathcal{K}_{\varepsilon}} B(A, r) \subset \mathcal{J}_{\varepsilon, r} \subset \bigcup_{A \in \mathcal{K}_{\varepsilon}} B\left(A, c_{0} r\right) .
$$

The structure $\mathcal{S}_{\varepsilon, r}$ is included in $\Omega_{\varepsilon}$.

The space of all admissible displacements is denoted $\mathbf{V}_{\varepsilon, r}$

$$
\mathbf{V}_{\varepsilon, r}=\left\{u \in H^{1}\left(\mathcal{S}_{\varepsilon, r}\right)^{3} \quad \mid \quad \exists u^{\prime} \in H^{1}\left(\mathcal{S}_{\varepsilon, r}^{\prime}\right)^{3} \text { such that } u_{\mid \mathcal{S}_{\varepsilon, r}}^{\prime}=u \text { and } u^{\prime}=0 \text { in } \mathcal{S}_{\varepsilon, r}^{\prime} \backslash \overline{\mathcal{S}_{\varepsilon, r}}\right\}
$$

It means that the displacements belonging to $\mathbf{V}_{\varepsilon, r}$ "vanish" on a part $\Gamma_{\varepsilon, r}$ included in $\partial \mathcal{S}_{\varepsilon, r} \cap$ $\partial \Omega$.

We assume that $\mathcal{S}_{\varepsilon, r}$ is made of isotropic and homogeneous material.

For a displacement $u \in \mathbf{V}_{\varepsilon, r}$, we denote by $e$ the strain tensor (or symmetric gradient)

$$
e(u) \doteq \frac{1}{2}\left(\nabla u+(\nabla u)^{T}\right), \quad e_{i j}(u) \doteq \frac{1}{2}\left(\frac{\partial u_{i}}{\partial x_{j}}+\frac{\partial u_{j}}{\partial x_{i}}\right)
$$

We have two coordinate systems. The first one is the global Cartesian system $\left(x_{1}, x_{2}, x_{3}\right)$ and is related to the frame $\left(O ; \mathbf{e}_{1}, \mathbf{e}_{2}, \mathbf{e}_{3}\right)$. The second one is the local coordinate system $\left(s_{1}, s_{2}, s_{3}\right)$ defined for every beam and related to the frame $\left(\varepsilon \xi+\varepsilon \mathbf{A}_{2}^{\ell} ; \mathbf{t}_{1}^{\ell}, \mathbf{t}_{2}^{\ell}, \mathbf{t}_{3}^{\ell}\right)$, $\ell \in\{1, \ldots, m\}$. The orthonormal transformation matrix from the basis $\left(\mathbf{t}_{1}^{\ell}, \mathbf{t}_{2}^{\ell}, \mathbf{t}_{3}^{\ell}\right)$ to the basis $\left(\mathbf{e}_{1}, \mathbf{e}_{2}, \mathbf{e}_{3}\right)$ is $\mathbf{T}^{\ell}=\left(\mathbf{t}_{1}^{\ell}\left|\mathbf{t}_{2}^{\ell}\right| \mathbf{t}_{3}^{\ell}\right)$, this matrix belongs to $S O(3)$. 
Hence, for every displacement $v \in H^{1}\left(\mathcal{P}_{\varepsilon \ell, r}^{\xi}\right)$ a straightforward calculation gives

$$
\begin{aligned}
e(v)= & \frac{1}{2}\left(\nabla_{x} v+\left(\nabla_{x} v\right)^{T}\right)=\frac{1}{2} \mathbf{T}^{\ell}\left(\nabla_{s} v+\left(\nabla_{s} v\right)^{T}\right)\left(\mathbf{T}^{\ell}\right)^{T}=\frac{1}{2} \mathbf{T}^{\ell} e_{s}(v)\left(\mathbf{T}^{\ell}\right)^{T} \\
e_{s}(v)= & \left(\begin{array}{ccc}
\frac{\partial v}{\partial s_{1}} \cdot \mathbf{t}_{1}^{\ell} & \frac{1}{2}\left(\frac{\partial v}{\partial s_{2}} \cdot \mathbf{t}_{1}^{\ell}+\frac{\partial v}{\partial s_{1}} \cdot \mathbf{t}_{2}^{\ell}\right) & \frac{1}{2}\left(\frac{\partial v}{\partial s_{3}} \cdot \mathbf{t}_{1}^{\ell}+\frac{\partial v}{\partial s_{1}} \cdot \mathbf{t}_{3}^{\ell}\right) \\
* & \frac{\partial v}{\partial s_{2}} \cdot \mathbf{t}_{2}^{\ell} & \frac{1}{2}\left(\frac{\partial v}{\partial s_{3}} \cdot \mathbf{t}_{2}^{\ell}+\frac{\partial v}{\partial s_{2}} \cdot \mathbf{t}_{3}^{\ell}\right) \\
* & * & \frac{\partial v}{\partial s_{3}} \cdot \mathbf{t}_{3}^{\ell}
\end{array}\right) .
\end{aligned}
$$

Let $a_{i j k l}^{\varepsilon, r} \in L^{\infty}\left(\mathcal{S}_{\varepsilon, r}\right),(i, j, k, l) \in\{1,2,3\}^{4}$, be the components of the elasticity tensor. These functions satisfy the usual symmetry and positivity conditions

- $a_{i j k l}^{\varepsilon, r}=a_{j i k l}^{\varepsilon, r}=a_{k l i j}^{\varepsilon, r} \quad$ a.e. in $\mathcal{S}_{\varepsilon, r}$;

- for any $\tau \in M_{s}^{3}$, where $M_{s}^{3}$ is the space of $3 \times 3$ symmetric matrices, there exists $C_{0}>0$ (independent of $\varepsilon$ and $r$ ) such that

$$
a_{i j k l}^{\varepsilon, r} \tau_{i j} \tau_{k l} \geq C_{0} \tau_{i j} \tau_{i j} \quad \text { a.e. in } \quad \mathcal{S}_{\varepsilon, r}
$$

The coefficients $a_{i j k l}^{\varepsilon}$ are given via the functions $a_{i j k l} \in L^{\infty}(\mathcal{S} \times D)$

$$
\begin{aligned}
& a_{i j k l}^{\varepsilon, r}(x)=a_{i j k l}^{\varepsilon, r}\left(\varepsilon \xi+\varepsilon A^{\ell}+s_{1} \mathbf{t}_{1}^{\ell}+s_{2} \mathbf{t}_{2}^{\ell}+s_{3} \mathbf{t}_{3}^{\ell}\right)=a_{i j k l}\left(A^{\ell}+\frac{s_{1}}{\varepsilon}, \frac{s_{2}}{r}, \frac{s_{3}}{r}\right) \\
& \text { for a.e. } x=\varepsilon \xi+\varepsilon A^{\ell}+s_{1} \mathbf{t}_{1}^{\ell}+s_{2} \mathbf{t}_{2}^{\ell}+s_{3} \mathbf{t}_{3}^{\ell} \text { in } \mathcal{P}_{\ell, r}^{\xi}, \quad \ell \in\{1, \ldots, m\}, \quad \xi \in \Xi_{\varepsilon} .
\end{aligned}
$$

The constitutive law for the material occupying the domain $\mathcal{S}_{\varepsilon, r}$ is given by the relation between the linearized strain tensor and the stress tensor

$$
\sigma_{i j}(u) \doteq a_{i j k l}^{\varepsilon, r} e_{s, k l}(u), \quad \forall u \in \mathbf{V}_{\varepsilon, r} .
$$

The unknown displacement $u_{\varepsilon}{ }^{1}: \mathcal{S}_{\varepsilon, r} \rightarrow \mathbb{R}^{3}$ is the solution to the linearized elasticity system:

$$
\begin{cases}\nabla \cdot \sigma\left(u_{\varepsilon}\right)=-f_{\varepsilon} & \text { in } \quad \mathcal{S}_{\varepsilon, r} \\ u_{\varepsilon}=0 & \text { on } \quad \Gamma_{\varepsilon, r} \cap \partial \mathcal{S}_{\varepsilon, r} \\ \sigma\left(u_{\varepsilon}\right) \cdot v_{\varepsilon}=0 & \text { on } \quad \partial \mathcal{S}_{\varepsilon, r} \backslash \Gamma_{\varepsilon, r}\end{cases}
$$

where $v_{\varepsilon}$ is the outward normal vector to $\partial \mathcal{S}_{\varepsilon, r} \backslash \Gamma, f_{\varepsilon}$ is the density of volume forces.

The variational formulation of problem (5.7) is

$$
\left\{\begin{array}{l}
\text { Find } u_{\varepsilon} \in \mathbf{V}_{\varepsilon, r} \text { such that, } \\
\int_{\mathcal{S}_{\varepsilon, r}} \sigma\left(u_{\varepsilon}\right): e(v) d x=\int_{\mathcal{S}_{\varepsilon, r}} f_{\varepsilon} \cdot v d x, \quad \forall v \in \mathbf{V}_{\varepsilon, r} .
\end{array}\right.
$$

\subsection{Final Decomposition of the Displacements of a Periodic Beam Stable Structure as a 3D-Like Domain}

Let $u$ be a displacement belonging to $\mathbf{V}_{\varepsilon, r}$. As proved in [14], we can decompose $u$ as the sum of an elementary displacement and a warping.

\footnotetext{
${ }^{1}$ Of course, the solution to this problem depends on $\varepsilon$ and $r$, but for simplicity, we omit the index $r$. The same holds for the applied forces $f_{\varepsilon}$ and for every function which in fact depends on both indexes.
} 
The decompositions introduced in Sect. 4, the estimates of Lemma 5 lead to the following estimates:

Lemma 7 For every $u \in \mathbf{V}_{\varepsilon, r}$ the following estimates hold:

$$
\begin{aligned}
& \|\bar{u}\|_{L^{2}\left(\mathcal{S}_{\varepsilon, r}\right)} \leq C r\|e(u)\|_{L^{2}\left(\mathcal{S}_{\varepsilon, r}\right)}, \quad\|\nabla \bar{u}\|_{L^{2}\left(\mathcal{S}_{\varepsilon, r}\right)} \leq C\|e(u)\|_{L^{2}\left(\mathcal{S}_{\varepsilon, r}\right)}, \\
& \left\|\frac{d \mathcal{R}}{d \mathbf{s}}\right\|_{L^{2}\left(\mathcal{S}_{\varepsilon}\right)} \leq \frac{C}{r^{2}}\|e(u)\|_{L^{2}\left(\mathcal{S}_{\varepsilon, r}\right)}, \quad\left\|\frac{d \mathcal{U}}{d \mathbf{s}}-\mathcal{R} \wedge \mathbf{t}_{1}\right\|_{L^{2}\left(\mathcal{S}_{\varepsilon}\right)} \leq \frac{C}{r}\|e(u)\|_{L^{2}\left(\mathcal{S}_{\varepsilon, r}\right)} .
\end{aligned}
$$

Moreover, one has

$$
\begin{aligned}
& \left\|\frac{d \overline{\mathcal{R}}}{d \mathbf{s}}\right\|_{L^{2}\left(\mathcal{S}_{\varepsilon}\right)} \leq \frac{C}{r^{2}}\|e(u)\|_{L^{2}\left(\mathcal{S}_{\varepsilon, r}\right)}, \quad\|\overline{\mathcal{R}}\|_{L^{2}\left(\mathcal{S}_{\varepsilon}\right)} \leq C \frac{\varepsilon}{r^{2}}\|e(u)\|_{L^{2}\left(\mathcal{S}_{\varepsilon, r}\right)}, \\
& \left\|\frac{d \overline{\mathcal{U}}}{d \mathbf{s}} \cdot \mathbf{t}_{1}\right\|_{L^{2}\left(\mathcal{S}_{\varepsilon}\right)} \leq \frac{C}{r}\|e(u)\|_{L^{2}\left(\mathcal{S}_{\varepsilon, r}\right)}, \quad\left\|\overline{\mathcal{U}} \cdot \mathbf{t}_{1}\right\|_{L^{2}\left(\mathcal{S}_{\varepsilon}\right)} \leq C \frac{\varepsilon}{r}\|e(u)\|_{L^{2}\left(\mathcal{S}_{\varepsilon, r}\right)}, \\
& \left\|\frac{d \overline{\mathcal{U}}}{d \mathbf{s}}\right\|_{L^{2}\left(\mathcal{S}_{\varepsilon}\right)} \leq C \frac{\varepsilon}{r^{2}}\|e(u)\|_{L^{2}\left(\mathcal{S}_{\varepsilon, r}\right)}, \quad\|\overline{\mathcal{U}}\|_{L^{2}\left(\mathcal{S}_{\varepsilon}\right)} \leq C \frac{\varepsilon^{2}}{r^{2}}\|e(u)\|_{L^{2}\left(\mathcal{S}_{\varepsilon, r}\right)}, \\
& \left\|\frac{d \mathcal{U}^{h}}{d \mathbf{S}}-\mathcal{R}^{h} \wedge \mathbf{t}_{1}\right\|_{L^{2}\left(\mathcal{S}_{\varepsilon}\right)} \leq C \frac{\varepsilon}{r^{2}}\|e(u)\|_{L^{2}\left(\mathcal{S}_{\varepsilon, r}\right)}, \\
& \left\|\frac{d \mathcal{R}^{h}}{d \mathbf{s}}\right\|_{L^{2}\left(\mathcal{S}_{\varepsilon}\right)}+\frac{1}{r}\left\|\frac{d \mathcal{U}^{h}}{d \mathbf{s}} \cdot \mathbf{t}_{1}\right\|_{L^{2}\left(\mathcal{S}_{\varepsilon}\right)} \leq \frac{C}{r^{2}}\|e(u)\|_{L^{2}\left(\mathcal{S}_{\varepsilon, r}\right)} .
\end{aligned}
$$

Proof We apply Lemma 5 to the structure $\varepsilon\left(\xi+\mathcal{S}_{1, \mathfrak{r}}\right)$. Replacing $\mathfrak{r}$ by $\frac{r}{\varepsilon}$ and then summing over all $\xi \in \Xi_{\varepsilon}$ give the estimates (5.9) and (5.10).

Let $u$ be in $H^{1}\left(\mathcal{S}_{\varepsilon, r}\right)^{3}$. In Lemma 5 replace $\mathcal{S}_{1, \mathfrak{r}}$ by $\varepsilon\left(\xi+\mathcal{S}_{r / \varepsilon}\right)$, with $\xi \in \Xi_{\varepsilon}$, and let $\mathbf{r}_{\varepsilon \xi}$ be a rigid displacement given by this lemma

$$
\mathbf{r}_{\varepsilon \xi}(x)=\mathbf{a}(\varepsilon \xi)+\mathbf{b}(\varepsilon \xi) \wedge(x-\varepsilon G-\varepsilon \xi), \quad \forall x \in \mathbb{R}^{3}
$$

One has

$$
\left\{\begin{array}{l}
\left\|\mathcal{U}^{h}-\mathbf{r}_{\varepsilon \xi}\right\|_{L^{2}(\varepsilon(\xi+\mathcal{S}))} \leq \frac{C \varepsilon}{r}\|e(u)\|_{L^{2}\left(\varepsilon\left(\xi+\mathcal{S}_{r / \varepsilon}\right)\right)}, \\
\left\|\frac{d \mathcal{U}^{h}}{d \mathbf{s}}-\mathbf{b}(\varepsilon \xi) \wedge \mathbf{t}_{1}\right\|_{L^{2}(\varepsilon(\xi+\mathcal{S}))} \leq \frac{C}{r}\|e(u)\|_{L^{2}\left(\varepsilon\left(\xi+\mathcal{S}_{r / \varepsilon}\right)\right)}
\end{array}\right.
$$

and

$$
\left\|\mathcal{R}^{h}-\mathbf{b}(\varepsilon \xi)\right\|_{L^{2}(\varepsilon(\xi+\mathcal{S}))} \leq C \frac{\varepsilon}{r^{2}}\|e(u)\|_{L^{2}\left(\varepsilon\left(\xi+\mathcal{S}_{r / \varepsilon}\right)\right)}
$$

Recall that if $\xi$ belongs to $\boldsymbol{\Xi}_{\varepsilon, i}$, the domains $\varepsilon\left(\xi+\mathcal{S}_{r / \varepsilon}\right)$ and $\varepsilon\left(\xi+\mathbf{e}_{i}+\mathcal{S}_{r / \varepsilon}\right), i \in\{1,2,3\}$, are included in $\mathcal{S}_{\varepsilon, r}$. Then, applying estimates (4.7) in Lemma 6 to the structure $\varepsilon\left(\xi+\mathcal{S}_{r / \varepsilon}\right)$ 
we obtain

$$
\left\{\begin{array}{l}
\sum_{1=1}^{3} \sum_{\xi \in \Xi_{\varepsilon, i}}\left|\mathbf{b}\left(\varepsilon \xi+\varepsilon \mathbf{e}_{i}\right)-\mathbf{b}(\varepsilon \xi)\right|^{2} \varepsilon^{3} \leq C \frac{\varepsilon^{2}}{r^{2}}\|e(u)\|_{L^{2}\left(\mathcal{S}_{\varepsilon, r}\right)}^{2}, \\
\sum_{i=1}^{3} \sum_{\xi \in \Xi_{\varepsilon, i}}\left|\mathbf{a}\left(\varepsilon \xi+\varepsilon \mathbf{e}_{i}\right)-\mathbf{a}(\varepsilon \xi)-\varepsilon \mathbf{b}\left(\varepsilon \xi+\varepsilon \mathbf{e}_{i}\right) \wedge \mathbf{e}_{i}\right|^{2} \varepsilon^{3} \leq C \frac{\varepsilon^{4}}{r^{2}}\|e(u)\|_{L^{2}\left(\mathcal{S}_{\varepsilon, r}\right)}^{2} .
\end{array}\right.
$$

Set

$$
\mathcal{U}(\varepsilon \xi)=\mathbf{a}(\varepsilon \xi), \quad \mathcal{R}(\varepsilon \xi)=\mathbf{b}(\varepsilon \xi), \quad \text { for every } \xi \in \Xi_{\varepsilon}
$$

Now, define

$-\mathcal{U}$ (resp. $\mathcal{R}$ ) in the cell $\varepsilon(\xi+\bar{Y}), \xi \in \widehat{\Xi}_{\varepsilon}$, as the $Q_{1}$ interpolate of its values on the vertices of this parallelotope.

$$
\mathcal{U}, \mathcal{R} \in W^{1, \infty}\left(\widehat{\Omega}_{\varepsilon}\right)^{3},
$$

- $\mathbf{a}$ (resp. b) as a piecewise constant function, equals to $\mathbf{a}(\varepsilon \xi)(\operatorname{resp} . \mathbf{b}(\varepsilon \xi))$ in the cell $\varepsilon(\xi+Y), \xi \in \Xi_{\varepsilon}$.

$$
\mathbf{a}, \mathbf{b} \in L^{\infty}\left(\Omega_{\varepsilon}\right)^{3} .
$$

We remind the following classical results ([10, Lemmas 5.22 and 5.35] and [16, Lemmas 5.2 and 5.3]):

Lemma 8 Let $\Omega$ be a bounded domain in $\mathbb{R}^{N}$ with Lipschitz boundary. There exists $\delta_{0}>0$ such that for all $\delta \in\left(0, \delta_{0}\right]$ the sets $\Omega_{\delta}^{\text {int }}=\{x \in \Omega \mid \operatorname{dist}(x, \partial \Omega)>\delta\}$ are uniformly Lipschitz.

Lemma 9 Let $\Psi$ be a function defined on $\Xi_{\varepsilon}$ and extended using the classical $Q_{1}$ interpolation procedure in a function denoted $\Psi$ and belonging to $W^{1, \infty}\left(\widehat{\Omega}_{\varepsilon}\right)$ then we have

$$
\begin{aligned}
& \varepsilon^{3} \sum_{\xi \in \Xi_{\varepsilon}^{i n t}}|\Psi(\xi)|^{2} \leq\|\Psi\|_{L^{2}\left(\Omega_{\varepsilon}^{i n t}\right)}^{2}, \\
& \sum_{\xi \in \Xi_{\varepsilon}}|\Psi(\xi)|^{2} \leq C\left(\sum_{\xi \in \Xi_{\varepsilon}^{i n t}}|\Psi(\xi)|^{2}+\sum_{i=1}^{3} \sum_{\xi \in \Xi_{\varepsilon, i}}\left|\Psi\left(\xi+\mathbf{e}_{i}\right)-\Psi(\xi)\right|^{2}\right) .
\end{aligned}
$$

Proposition 1 Let $\mathcal{S}$ be a 3-PSS. For every displacement $u \in H^{1}\left(\mathcal{S}_{\varepsilon, r}\right)^{3}$, one has

$$
\begin{aligned}
& \|\nabla \mathcal{R}\|_{L^{2}\left(\Omega_{\varepsilon}^{i n t}\right)} \leq \frac{C}{r}\|e(u)\|_{L^{2}\left(\mathcal{S}_{\varepsilon, r}\right)}, \\
& \left\|\frac{\partial \mathcal{U}}{\partial x_{i}}-\mathcal{R} \wedge \mathbf{e}_{i}\right\|_{L^{2}\left(\Omega_{\varepsilon}^{i n t}\right)} \leq C \frac{\varepsilon}{r}\|e(u)\|_{L^{2}\left(\mathcal{S}_{\varepsilon, r}\right)}, \quad i \in\{1,2,3\} \\
& \|e(\mathcal{U})\|_{L^{2}\left(\Omega_{\varepsilon}^{i n t}\right)} \leq C \frac{\varepsilon}{r}\|e(u)\|_{L^{2}\left(\mathcal{S}_{\varepsilon, r}\right)} .
\end{aligned}
$$

Moreover, there exists a rigid displacement $\mathbf{r}$ such that

$$
\|\mathcal{U}-\mathbf{r}\|_{H^{1}\left(\Omega_{\varepsilon}^{i n t}\right)} \leq C \frac{\varepsilon}{r}\|e(u)\|_{L^{2}\left(\mathcal{S}_{\varepsilon, r}\right)} .
$$


Proof The estimates $(5.13)_{1,2}$ and Lemma 9 yield

$$
\begin{aligned}
& \|\nabla \boldsymbol{R}\|_{L^{2}\left(\Omega_{\varepsilon}^{i n t}\right)} \leq\|\nabla \mathcal{R}\|_{L^{2}\left(\widehat{\Omega}_{\varepsilon}\right)} \leq C \frac{\varepsilon}{r^{2}}\|e(u)\|_{L^{2}\left(\mathcal{S}_{\varepsilon, r}\right)}, \\
& \left\|\frac{\partial \mathcal{U}}{\partial x_{i}}-\mathcal{R} \wedge \mathbf{e}_{i}\right\|_{L^{2}\left(\Omega_{\varepsilon}^{i n t}\right)} \leq\left\|\frac{\partial \mathcal{U}}{\partial x_{i}}-\mathcal{R} \wedge \mathbf{e}_{i}\right\|_{L^{2}\left(\widehat{\Omega}_{\varepsilon}\right)} \leq C \frac{\varepsilon}{r}\|e(u)\|_{L^{2}\left(\mathcal{S}_{\varepsilon, r}\right)}, \quad i \in\{1,2,3\} .
\end{aligned}
$$

And (5.16) $)_{1,2}$ are proved. From which we get

$$
\begin{aligned}
& \left\|\frac{\partial \mathcal{U}}{\partial x_{i}} \cdot \mathbf{e}_{j}+\frac{\partial \mathcal{U}}{\partial x_{j}} \cdot \mathbf{e}_{i}\right\|_{L^{2}\left(\Omega_{\varepsilon}^{i n t}\right)} \leq\left\|\frac{\partial \mathcal{U}}{\partial x_{i}} \cdot \mathbf{e}_{j}+\frac{\partial \mathcal{U}}{\partial x_{j}} \cdot \mathbf{e}_{i}\right\|_{L^{2}\left(\widehat{\Omega}_{\varepsilon}\right)} \leq C \frac{\varepsilon}{r}\|e(u)\|_{L^{2}\left(\mathcal{S}_{\varepsilon, r}\right)}, \\
& \forall(i, j) \in\{1,2,3\}^{2},
\end{aligned}
$$

which also read $(5.16)_{3}$. Lemma 8 allows to apply the 3D-Korn inequality in the domain $\Omega_{\varepsilon}^{i n t}$ using estimate $(5.16)_{3}$. That gives (5.17).

Proposition 2 Let $\mathcal{S}$ be a 3-PSS. For every $u$ in $\mathbf{V}_{\varepsilon, r}$, the following estimates of the elementary displacement holds:

$$
\begin{array}{ll}
\|\mathcal{U}\|_{L^{2}\left(\mathcal{S}_{\varepsilon}\right)} \leq \frac{C}{r}\left(1+\frac{\varepsilon^{2}}{r}\right)\|e(u)\|_{L^{2}\left(\mathcal{S}_{\varepsilon, r}\right)}, & \left\|\frac{d \mathcal{U}}{d \mathbf{s}}\right\|_{L^{2}\left(\mathcal{S}_{\varepsilon}\right)} \leq C \frac{\varepsilon}{r^{2}}\|e(u)\|_{L^{2}\left(\mathcal{S}_{\varepsilon, r}\right)}, \\
\|\mathcal{R}\|_{L^{2}\left(\mathcal{S}_{\varepsilon}\right)}+\varepsilon\left\|\frac{d \mathcal{R}}{d \mathbf{s}}\right\|_{L^{2}\left(\mathcal{S}_{\varepsilon}\right)} \leq C \frac{\varepsilon}{r^{2}}\|e(u)\|_{L^{2}\left(\mathcal{S}_{\varepsilon, r}\right)}, & \\
\left\|U^{e}\right\|_{L^{2}\left(\mathcal{S}_{\varepsilon, r}\right)} \leq C\left(1+\frac{\varepsilon^{2}}{r}\right)\|e(u)\|_{L^{2}\left(\mathcal{S}_{\varepsilon, r}\right)}, \quad & \left\|\nabla U^{e}\right\|_{L^{2}\left(\mathcal{S}_{\varepsilon, r}\right)} \leq C \frac{\varepsilon}{r}\|e(u)\|_{L^{2}\left(\mathcal{S}_{\varepsilon, r}\right)} .
\end{array}
$$

Moreover, one has the Korn type inequalities

$$
\|u\|_{L^{2}\left(\mathcal{S}_{\varepsilon, r}\right)} \leq C\left(1+\frac{\varepsilon^{2}}{r}\right)\|e(u)\|_{L^{2}\left(\mathcal{S}_{\varepsilon, r}\right)}, \quad\|\nabla u\|_{L^{2}\left(\mathcal{S}_{\varepsilon, r}\right)} \leq C \frac{\varepsilon}{r}\|e(u)\|_{L^{2}\left(\mathcal{S}_{\varepsilon, r}\right)}
$$

Proof This proposition is a consequence of Proposition 1 and two lemmas postponed in Appendix A.

\subsection{Assumptions on the Applied Forces}

We distinguish two types of applied forces. The first ones are applied in the beams (between the junctions) and the second ones are applied in the junctions.

$\star$ The applied forces $\mathbf{f}_{\varepsilon}$ in the set of beams $\bigcup_{\xi \in \Xi_{\varepsilon}} \bigcup_{\ell=1}^{m} \mathcal{P}_{\varepsilon \ell, r}^{\xi}$.

For simplicity, we choose these applied forces constant in the cross-sections and equal to

$$
\mathbf{f}_{\varepsilon}=\frac{\varepsilon}{r+\varepsilon^{2}} \mathbf{f}_{\mathcal{S}_{\varepsilon}} \quad \text { a.e. in } \bigcup_{\xi \in \Xi_{\varepsilon}} \bigcup_{\ell=1}^{m} \mathcal{P}_{\varepsilon \ell, r}^{\xi}
$$

$\star$ The applied forces $F_{r, \mathcal{K}_{\varepsilon}}$ in the junctions. 
These forces are defined in the balls centered in the nodes with radius $r$

$$
F_{r, \mathcal{K}_{\varepsilon}}=\sum_{A \in \mathcal{K}_{\varepsilon}} \frac{\varepsilon^{2}}{r^{2}} F(A) \mathbf{1}_{B(A, r)}+\sum_{A \in \mathcal{K}_{\varepsilon}} \frac{\varepsilon}{r^{3}} G(A) \wedge(x-A) \mathbf{1}_{B(A, r)},
$$

Lemma 10 Taking the applied forces as

$$
f_{\varepsilon}=\sum_{A \in \mathcal{K}_{\varepsilon}}\left[\frac{\varepsilon^{2}}{r^{2}} F(A)+\frac{\varepsilon}{r^{3}} G(A) \wedge(x-A)\right] \mathbf{1}_{B(A, r)}+\frac{\varepsilon}{r+\varepsilon^{2}} \mathbf{f}_{\mid \mathcal{S}_{\varepsilon}} \mathbf{1}_{\cup_{\xi \in \Xi_{\varepsilon}} \cup_{\ell=1}^{m} \mathcal{P}_{\varepsilon \ell, r}^{\xi}}
$$

where $(\mathbf{f}, F, G) \in\left(C(\bar{\Omega})^{3}\right)^{3}$ and where $\mathbf{1}_{\mathcal{O}}$ is the characteristic function of the set $\mathcal{O}$, we obtain

$$
\left|\int_{\mathcal{S}_{\varepsilon, r}} f_{\varepsilon} \cdot u d x\right| \leq C\left(\|\mathbf{f}\|_{L^{\infty}(\Omega)}+\|F\|_{L^{\infty}(\Omega)}+\|G\|_{L^{\infty}(\Omega)}\right)\|e(u)\|_{L^{2}\left(\mathcal{S}_{\varepsilon, r}\right)}, \quad \forall u \in \mathbf{V}_{\varepsilon, r} .
$$

Proof The proof is postponed in Appendix B.

As a consequence of the above lemma one obtains

Proposition 3 The solution $u_{\varepsilon}$ to the problem (5.8) satisfies

$$
\left\|e\left(u_{\varepsilon}\right)\right\|_{L^{2}\left(\mathcal{S}_{\varepsilon, r}\right)} \leq C\left(\|\mathbf{f}\|_{L^{\infty}(\Omega)}+\|F\|_{L^{\infty}(\Omega)}+\|G\|_{L^{\infty}(\Omega)}\right) .
$$

Proof In order to obtain apriori estimate of $u_{\varepsilon}$, we test (5.8) with $v=u_{\varepsilon}$. From (5.21), we obtain

$$
\left\|e\left(u_{\varepsilon}\right)\right\|_{L^{2}\left(\mathcal{S}_{\varepsilon, r}\right)}^{2} \leq C\left(\|\mathbf{f}\|_{L^{\infty}(\Omega)}+\|F\|_{L^{\infty}(\Omega)}+\|G\|_{L^{\infty}(\Omega)}\right)\left\|e\left(u_{\varepsilon}\right)\right\|_{L^{2}\left(\mathcal{S}_{\varepsilon, r}\right)},
$$

which leads to (5.22).

\section{The Unfolding Operators}

The classical unfolding operator $\mathcal{T}_{\varepsilon}$ is developed in [9, 10]. Here, we will use similar operators $\mathcal{T}_{\varepsilon}^{\text {ext }}, \mathcal{T}_{\varepsilon}^{\mathcal{S}}, \mathcal{T}_{\varepsilon}^{b, \ell}$ in the context of the domains $\Omega_{\varepsilon}, \mathcal{S}_{\varepsilon}$ and $\mathcal{S}_{\varepsilon, r}$.

Definition 6 (Classical unfolding-operator) For a measurable function $\phi$ on $\Omega$, the unfolding operator $\mathcal{T}_{\varepsilon}$ is defined as follows:

$$
\begin{aligned}
& \mathcal{T}_{\varepsilon}(\phi)(x, y)=\phi\left(\varepsilon\left[\frac{x}{\varepsilon}\right]+\varepsilon y\right) \text { for a.e. }(x, y) \in \widetilde{\Omega}_{\varepsilon} \times Y, \\
& \mathcal{T}_{\varepsilon}(\phi)(x, y)=0 \quad \text { for a.e. }(x, y) \in\left(\Omega \backslash \widetilde{\Omega}_{\varepsilon}\right) \times Y .
\end{aligned}
$$

Definition 7 (Unfolding-operator) For a measurable function $\phi$ on $\Omega_{\varepsilon}$, the unfolding operator $\mathcal{T}_{\varepsilon}^{\text {ext }}$ is defined as follows:

$$
\mathcal{T}_{\varepsilon}^{\text {ext }}(\phi)(x, y)=\phi\left(\varepsilon\left[\frac{x}{\varepsilon}\right]+\varepsilon y\right) \quad \text { for a.e. }(x, y) \in \Omega_{\varepsilon} \times Y .
$$


Lemma 11 Let $\phi$ be in $L^{p}\left(\Omega_{\varepsilon}\right), p \in[1,+\infty)$. One has

$$
\left\|\mathcal{T}_{\varepsilon}^{\text {ext }}(\phi)-\mathcal{T}_{\varepsilon}(\phi)\right\|_{L^{p}(\Omega \times Y)} \leq\|\phi\|_{L^{p}\left(\Omega_{\varepsilon}^{b l}\right)}
$$

where

$$
\Omega_{\varepsilon}^{b l} \doteq\left\{x \in \Omega_{\varepsilon} \mid \operatorname{dist}(x, \partial \Omega) \leq \varepsilon \sqrt{3}\right\}
$$

Proof Inequality (6.1) is an immediate consequence of the definitions of these operators.

As a consequence of the above lemma, the properties of the operator $\mathcal{T}_{\varepsilon}^{\text {ext }}$ are similar to those of the classical unfolding operator $\mathcal{T}_{\varepsilon}$. For the main properties of the unfolding operator $\mathcal{T}_{\varepsilon}$, we refer the reader to [10, Chap. 1].

Below, we introduce two new unfolding operators. The first one is used for the centerlines of beams and the second one is used for the small beams (it concerns the reduction of dimension).

In the definitions below, $\varepsilon\left[\frac{x}{\varepsilon}\right]$ represents a macroscopic coordinate (the same coordinate for all the points in the cell $\varepsilon\left[\frac{x}{\varepsilon}\right]+\varepsilon Y$ ) while $\mathbf{S}$ is the coordinate of a point belonging to $\mathcal{S}$. Hence, $\varepsilon\left[\frac{x}{\varepsilon}\right]+\varepsilon \mathbf{S}$ represents the coordinate of a point belonging to $\mathcal{S}_{\varepsilon}$. In order to get a map $(x, \mathbf{S}) \longmapsto \varepsilon\left[\frac{x}{\varepsilon}\right]+\varepsilon \mathbf{S}$ almost one to one, we have to restrict the set $\mathcal{S}$. This is why from now on, to introduce the unfolding operator, in lieu of $\mathcal{S}$ we consider the set

$$
\mathcal{S} \cap[0,1)^{3} .
$$

For simplicity we still refer to it as $\mathcal{S}$. The set of new nodes is always denoted $\mathcal{K}$ and the number of beams of $\mathcal{S}$ is still denoted $m$.

Definition 8 (Centerlines unfolding) For a measurable function $\phi$ on $\mathcal{S}_{\varepsilon}$, the unfolding operator $\mathcal{T}_{\bar{\varepsilon}}^{\mathcal{S}}$ is defined as follows:

$$
\mathcal{T}_{\varepsilon}^{\mathcal{S}}(\phi)(x, \mathbf{S})=\phi\left(\varepsilon\left[\frac{x}{\varepsilon}\right]+\varepsilon \mathbf{S}\right) \text { for a.e. }(x, \mathbf{S}) \in \Omega_{\varepsilon} \times \mathcal{S} .
$$

Definition 9 (Beams unfolding) For a measurable function $u$ on $\mathcal{S}_{\varepsilon, r}$, the unfolding operator $\mathcal{T}_{\varepsilon}^{b, \ell}$ is defined as follows $(\ell \in\{1, \ldots, m\})$ :

$\mathcal{T}_{\varepsilon}^{b, \ell}(u)(x, \widehat{S})=u\left(\varepsilon\left[\frac{x}{\varepsilon}\right]+\varepsilon \mathbf{A}^{\ell}+\varepsilon S_{1} \mathbf{t}_{1}^{\ell}+r S_{2} \mathbf{t}_{2}^{\ell}+r S_{3} \mathbf{t}_{3}^{\ell}\right) \quad$ for a.e. $(x, \widehat{S}) \in \Omega_{\varepsilon} \times\left(0, l_{\ell}\right) \times D$,

where $\widehat{S}=\left(S_{1}, S_{2}, S_{3}\right), \mathbf{A}^{\ell}$ is an extremity of the segment $\gamma_{\ell} \subset \mathcal{S}$ and $D=D_{1}$ is the disc of radius 1 .

Let $\phi$ be measurable on $\mathcal{S}_{\varepsilon}$, one has

$$
\mathcal{T}_{\varepsilon}^{\mathcal{S}}(\phi)(x, \mathbf{S})=\phi\left(\varepsilon\left[\frac{x}{\varepsilon}\right]+\varepsilon \mathbf{S}\right)=\phi\left(\varepsilon\left[\frac{x}{\varepsilon}\right]+\varepsilon \mathbf{A}^{\ell}+\varepsilon S_{1} \mathbf{t}_{1}^{\ell}\right)=\mathcal{T}_{\varepsilon}^{b, \ell}(\phi)\left(x,\left(\widehat{S_{1}, 0,0}\right)\right)
$$

for a.e. $\left(x, S_{1}\right) \in \Omega_{\varepsilon} \times\left(0, l_{\ell}\right)$. 
Lemma 12 (Properties of the operators $\mathcal{T}_{\varepsilon}^{\mathcal{S}}$ and $\mathcal{T}_{\varepsilon}^{b, \ell}$ ) For every $\phi \in L^{1}\left(\mathcal{S}_{\varepsilon}\right)$

$$
\int_{\Omega_{\varepsilon} \times \mathcal{S}} \mathcal{T}_{\varepsilon}^{\mathcal{S}}(\phi)(x, \mathbf{S}) d \mathbf{S} d x=\varepsilon^{2} \int_{\mathcal{S}_{\varepsilon}} \phi(x) d x .
$$

For every $\phi \in L^{2}\left(\mathcal{S}_{\varepsilon}\right)$

$$
\left\|\mathcal{T}_{\varepsilon}^{\mathcal{S}}(\phi)\right\|_{L^{2}\left(\Omega_{\varepsilon} \times \mathcal{S}\right)}=\varepsilon\|\phi\|_{L^{2}\left(\mathcal{S}_{\varepsilon}\right)} .
$$

For every $\phi$ in $H^{1}\left(\mathcal{S}_{\varepsilon}\right)$

$$
\frac{\partial \mathcal{T}_{\varepsilon}^{\mathcal{S}}(\phi)}{\partial \mathbf{S}}(x, \mathbf{S})=\varepsilon \mathcal{T}_{\varepsilon}^{\mathcal{S}}\left(\frac{d \phi}{d \mathbf{s}}\right)(x, \mathbf{S}) \quad \text { for a.e. }(x, \mathbf{S}) \in \Omega_{\varepsilon} \times \mathcal{S} .
$$

For every $\psi$ in $L^{2}\left(\mathcal{S}_{\varepsilon, r}\right)$

$$
\left\|\mathcal{T}_{\varepsilon}^{b, \ell}(\psi)\right\|_{L^{2}\left(\Omega_{\varepsilon} \times \gamma_{\ell} \times D\right)} \leq C \frac{\varepsilon}{r}\|\psi\|_{L^{2}\left(\mathcal{S}_{\varepsilon, r}\right)} \quad \text { for all } \ell \in\{1, \ldots, m\} .
$$

For every $\psi$ in $L^{1}\left(\mathcal{S}_{\varepsilon, r}\right)$

$$
\left|\sum_{\ell=1}^{m} \int_{\Omega_{\varepsilon} \times \gamma_{\ell} \times D} \frac{r^{2}}{\varepsilon^{2}} \mathcal{T}_{\varepsilon}^{b, \ell}(\psi)(x, \widehat{S}) d x d \widehat{S}-\int_{\mathcal{S}_{\varepsilon, r}} \psi(x) d x\right| \leq C\|\psi\|_{L^{1}\left(\mathcal{J}_{\varepsilon, r}\right)} .
$$

The constant only depends on $\mathcal{S}$.

For every $u$ in $H^{1}\left(\mathcal{S}_{\varepsilon, r}\right)(j \in\{2,3\}$ and $\ell \in\{1, \ldots, m\})$

$$
\begin{aligned}
& { }_{\varepsilon} \mathcal{T}_{\varepsilon}^{b, \ell}(\nabla u)(x, \widehat{S}) \cdot \mathbf{t}_{1}^{\ell}=\frac{\partial \mathcal{T}_{\varepsilon}^{b, \ell}(u)}{\partial S_{1}}(x, \widehat{S}), \\
& r \mathcal{T}_{\varepsilon}^{b, \ell}(\nabla u)(x, \widehat{S}) \cdot \mathbf{t}_{j}^{\ell}=\frac{\partial \mathcal{T}_{\varepsilon}^{b, \ell}(u)}{\partial S_{j}}(x, \widehat{S}), \\
& \text { for a.e. }(x, \widehat{S}) \in \Omega_{\varepsilon} \times\left(0, l_{\ell}\right) \times D \text {. }
\end{aligned}
$$

Proof We prove (6.2) and (6.3). Let $\phi$ be in $L^{1}\left(\mathcal{S}_{\varepsilon}\right)$

$$
\begin{aligned}
\int_{\Omega_{\varepsilon} \times \mathcal{S}} \mathcal{T}_{\varepsilon}^{S}(\phi)(x, \mathbf{S}) d \mathbf{S} d x & =\sum_{\ell=1}^{m} \int_{\Omega_{\varepsilon} \times \gamma_{\ell}} \mathcal{T}_{\varepsilon}^{S}(\phi)\left(x, \mathbf{A}^{\ell}+S_{1} \mathbf{t}_{1}^{\ell}\right) d x d S_{1} \\
& =\sum_{\ell=1}^{m} \sum_{\xi \in \Xi_{\varepsilon}}|\varepsilon \xi+\varepsilon Y| \int_{0}^{l_{\ell}} \phi\left(\varepsilon \xi+\varepsilon \mathbf{A}^{\ell}+\varepsilon t\right) d t \\
& =\sum_{\ell=1}^{m} \sum_{\xi \in \Xi_{\varepsilon}} \varepsilon^{3} \int_{0}^{l_{\ell}} \phi\left(\varepsilon \xi+\varepsilon \mathbf{A}^{\ell}+\varepsilon t\right) d t=\varepsilon^{2} \int_{\mathcal{S}_{\varepsilon}} \phi(x) d x .
\end{aligned}
$$

We prove (6.6). For $u \in L^{1}\left(\mathcal{S}_{\varepsilon, r}\right)$ we have

$$
\begin{aligned}
& \int_{\Omega_{\varepsilon} \times \gamma_{\ell} \times D} \mathcal{T}_{\varepsilon}^{b, \ell}(u)(x, \widehat{S}) d x d \widehat{S} \\
& \quad=\sum_{\xi \in \Xi_{\varepsilon}} \int_{(\varepsilon \xi+\varepsilon Y) \times \gamma_{\ell} \times D} u\left(\varepsilon\left[\frac{x}{\varepsilon}\right]+\varepsilon \mathbf{A}^{\ell}+\varepsilon S_{1} \mathbf{t}_{1}^{\ell}+r S_{2} \mathbf{t}_{2}^{\ell}+r S_{3} \mathbf{t}_{3}^{\ell}\right) d x d \widehat{S}
\end{aligned}
$$




$$
\begin{aligned}
& =\sum_{\xi \in \Xi_{\varepsilon}} \int_{(\varepsilon \xi+\varepsilon Y) \times \gamma_{\ell} \times D} u\left(\varepsilon \xi+\varepsilon \mathbf{A}^{\ell}+\varepsilon S_{1} \mathbf{t}_{1}^{\ell}+r S_{2} \mathbf{t}_{2}^{\ell}+r S_{3} \mathbf{t}_{3}^{\ell}\right) d x d \widehat{S} \\
& =\sum_{\xi \in \Xi_{\varepsilon}}|\varepsilon \xi+\varepsilon Y| \int_{\gamma_{\ell} \times D} u\left(\varepsilon \xi+\varepsilon \mathbf{A}^{\ell}+\varepsilon S_{1} \mathbf{t}_{1}^{\ell}+r S_{2} \mathbf{t}_{2}^{\ell}+r S_{3} \mathbf{t}_{3}^{\ell}\right) d \widehat{S} \\
& =\varepsilon^{3} \sum_{\xi \in \Xi_{\varepsilon}} \int_{\gamma_{\ell} \times D} u\left(\varepsilon \xi+\varepsilon \mathbf{A}^{\ell}+\varepsilon S_{1} \mathbf{t}_{1}^{\ell}+r S_{2} \mathbf{t}_{2}^{\ell}+r S_{3} \mathbf{t}_{3}^{\ell}\right) d \widehat{S} .
\end{aligned}
$$

Now, replacing $\varepsilon \xi+\varepsilon \mathbf{A}^{\ell}+\varepsilon S_{1} \mathbf{t}_{1}^{\ell}+r S_{2} \mathbf{t}_{2}^{\ell}+r S_{3} \mathbf{t}_{3}^{\ell}$ by $x$ and taking into account that the matrix $\left(\mathbf{t}_{1}^{\ell}\left|\mathbf{t}_{2}^{\ell}\right| \mathbf{t}_{3}^{\ell}\right)$ belongs to $S O(3)$, we obtain

$$
\int_{\Omega_{\varepsilon} \times \gamma_{\ell} \times D} \mathcal{T}_{\varepsilon}^{b, \ell}(u)(x, \widehat{S}) d x d \widehat{S}=\frac{\varepsilon^{2}}{r^{2}} \sum_{\xi \in \Xi_{\varepsilon}} \int_{\left(\varepsilon \xi+\varepsilon \mathcal{P}_{\ell, r / \varepsilon}\right)} u(x) d x=\frac{\varepsilon^{2}}{r^{2}} \sum_{\xi \in \Xi_{\varepsilon}} \int_{\mathcal{P}_{\varepsilon \ell, r}^{\xi}} u(x) d x
$$

and (6.6) follows.

Properties (6.4)-(6.7) are direct consequences of the definitions of the unfolding operators.

Corollary 1 For every $\phi$ in $L^{2}\left(\mathcal{S}_{\varepsilon}\right), \ell \in\{1, \ldots, m\}$

$$
\left\|\mathcal{T}_{\varepsilon}^{b, \ell}(\phi)\right\|_{L^{2}\left(\Omega \times \gamma_{\ell} \times D\right)} \leq C \varepsilon\|\phi\|_{L^{2}\left(\mathcal{S}_{\varepsilon}\right)} \text {. }
$$

From now on, every function belonging to $L^{p}(\Omega)(p \in[1,+\infty])$ will be extended by 0 in $\Omega_{\varepsilon} \backslash \bar{\Omega}$.

Denote $Q_{1}(Y)$ the subspace of $W^{1, \infty}(Y)$ containing the functions which are the $Q_{1}$ interpolations of their values at the vertices of the parallelotope $\bar{Y}$.

Lemma 13 For every $\Phi$ in $W^{1, \infty}\left(\Omega_{\varepsilon}\right)$ satisfying

$$
\mathcal{T}_{\varepsilon}^{\text {ext }}(\Phi) \in L^{\infty}\left(\Omega ; Q_{1}(Y)\right)
$$

Then $\Phi_{\mid \mathcal{S}_{\varepsilon}}$ belongs to $W^{1, \infty}\left(\mathcal{S}_{\varepsilon}\right)$ and it satisfies

$$
\begin{aligned}
& \left\|\Phi_{\mid \mathcal{S}_{\varepsilon}}\right\|_{L^{2}\left(\mathcal{S}_{\varepsilon}\right)} \leq \frac{C}{\varepsilon}\|\Phi\|_{L^{2}\left(\Omega_{\varepsilon}\right)}, \\
& \frac{d \Phi_{\mid \mathcal{S}_{\varepsilon}}}{d \mathbf{s}}=\nabla \Phi_{\varepsilon} \cdot \mathbf{t}_{1} \quad \text { a.e. in } \mathcal{S}_{\varepsilon} \quad \text { and } \quad\left\|\frac{d \Phi_{\mid \mathcal{S}_{\varepsilon}}}{d \mathbf{s}}\right\|_{L^{2}\left(\mathcal{S}_{\varepsilon}\right)} \leq \frac{C}{\varepsilon}\|\nabla \Phi\|_{L^{2}\left(\Omega_{\varepsilon}\right)} .
\end{aligned}
$$

Let $\left\{\Phi_{\varepsilon}\right\}_{\varepsilon}$ be a sequence of functions belonging to $W^{1, \infty}\left(\Omega_{\varepsilon}\right)$ satisfying (6.9) and

$$
\left\|\Phi_{\varepsilon}\right\|_{L^{2}\left(\Omega_{\varepsilon}\right)} \leq C
$$

then, up to a subsequence of $\{\varepsilon\}$, there exists $\Phi \in L^{2}(\Omega)$ such that

$$
\begin{aligned}
& \Phi_{\varepsilon} \rightarrow \Phi \quad \text { weakly in } L^{2}(\Omega), \\
& \mathcal{T}_{\varepsilon}^{\text {ext }}\left(\Phi_{\varepsilon}\right) \rightarrow \Phi \quad \text { weakly in } L^{2}\left(\Omega ; Q_{1}(Y)\right), \\
& \mathcal{T}_{\varepsilon}^{\text {ext }}\left(\Phi_{\varepsilon}\right)_{\mid \Omega \times \mathcal{S}}=\mathcal{T}_{\varepsilon}^{\mathcal{S}}\left(\Phi_{\varepsilon}\right) \rightarrow \Phi \quad \text { weakly in } L^{2}\left(\Omega ; H^{1}(\mathcal{S})\right) .
\end{aligned}
$$


Moreover, if one also has

$$
\left\|\nabla \Phi_{\varepsilon}\right\|_{L^{2}\left(\Omega_{\varepsilon}\right)} \leq C
$$

then $\Phi$ belongs to $H^{1}(\Omega)$ and

$$
\begin{aligned}
& \Phi_{\varepsilon} \rightarrow \Phi \quad \text { weakly in } H^{1}(\Omega), \\
& \mathcal{T}_{\varepsilon}^{\text {ext }}\left(\nabla \Phi_{\varepsilon}\right) \rightarrow \nabla \Phi \quad \text { weakly in } L^{2}(\Omega \times Y)^{3}, \\
& \mathcal{T}_{\varepsilon}^{\mathcal{S}}\left(\frac{d \Phi_{\varepsilon}}{d \mathbf{s}}\right)=\mathcal{T}_{\varepsilon}^{\text {ext }}\left(\nabla \Phi_{\varepsilon} \cdot \mathbf{t}_{1}\right)_{\mid \Omega \times \mathcal{S}} \rightarrow \nabla \Phi \cdot \mathbf{t}_{1} \quad \text { weakly in } L^{2}(\Omega \times \mathcal{S}) .
\end{aligned}
$$

Proof The proof is given in Appendix C.

First convergence results for sequences in $H^{1}\left(\mathcal{S}_{\varepsilon}\right)$.

Lemma 14 Let $\left\{\phi_{\varepsilon}\right\}_{\varepsilon}$ be a sequence of functions belonging to $H^{1}\left(\mathcal{S}_{\varepsilon}\right)$ satisfying

$$
\left\|\phi_{\varepsilon}\right\|_{L^{2}\left(\mathcal{S}_{\varepsilon}\right)}+\varepsilon\left\|\frac{d \phi_{\varepsilon}}{d \mathbf{s}}\right\|_{L^{2}\left(\mathcal{S}_{\varepsilon}\right)} \leq \frac{C}{\varepsilon} .
$$

Then, up to a subsequence, there exists $\widehat{\phi} \in L^{2}\left(\Omega ; H_{\text {per }}^{1}(\mathcal{S})\right)$ such that

$$
\mathcal{T}_{\varepsilon}^{\mathcal{S}}\left(\phi_{\varepsilon}\right) \rightarrow \widehat{\phi} \quad \text { weakly in } L^{2}\left(\Omega ; H^{1}(\mathcal{S})\right) \text {. }
$$

If we only have

$$
\left\|\phi_{\varepsilon}\right\|_{L^{2}\left(\mathcal{S}_{\varepsilon} \cap \Omega_{\varepsilon}^{i n t}\right)}+\varepsilon\left\|\frac{d \phi_{\varepsilon}}{d \mathbf{s}}\right\|_{L^{2}\left(\mathcal{S}_{\varepsilon} \cap \Omega_{\varepsilon}^{i n t}\right)} \leq \frac{C}{\varepsilon},
$$

then, up to a subsequence, there exists $\widehat{\phi} \in L^{2}\left(\Omega ; H_{p e r}^{1}(\mathcal{S})\right)$ such that

$$
\mathcal{T}_{\varepsilon}^{\mathcal{S}}\left(\phi_{\varepsilon}\right) \mathbf{1}_{\widehat{\Omega}_{\varepsilon}^{i n t} \times \mathcal{S}} \rightarrow \widehat{\phi} \quad \text { weakly in } L^{2}\left(\Omega ; H^{1}(\mathcal{S})\right) .
$$

Proof The proof is postponed in Appendix C.

Definition 10 The local average operator $\mathcal{M}_{\varepsilon}^{*}$ is defined from $L^{2}\left(\mathcal{S}_{\varepsilon}\right)$ to $L^{2}\left(\Omega_{\varepsilon}\right)$ as

$$
\mathcal{M}_{\varepsilon}^{*}(\phi)(x)=\frac{1}{|\mathcal{S}|} \int_{\mathcal{S}} \mathcal{T}_{\varepsilon}^{\mathcal{S}}(\phi)(x, \mathbf{S}) d \mathbf{S}, \quad \text { for a.e. } x \in \Omega_{\varepsilon}
$$

By convention the value of $\mathcal{M}_{\varepsilon}^{*}(\phi)$ on the cell $\varepsilon(\xi+Y)$ is simply denoted $\mathcal{M}_{\varepsilon}^{*}(\phi)(\varepsilon \xi)$.

A second lemma for sequences in $H^{1}\left(\mathcal{S}_{\varepsilon}\right)$.

Lemma 15 Let $\left\{\phi_{\varepsilon}\right\}_{\varepsilon}$ be a sequence of functions belonging to $H^{1}\left(\mathcal{S}_{\varepsilon}\right)$ satisfying

$$
\left\|\phi_{\varepsilon}\right\|_{H^{1}\left(\mathcal{S}_{\varepsilon}\right)} \leq \frac{C}{\varepsilon} .
$$

Then, up to a subsequence, there exists $(\Phi, \widehat{\phi}) \in H^{1}(\Omega) \times L^{2}\left(\Omega ; H_{p e r}^{1}(\mathcal{S})\right)$ such that

$$
\begin{aligned}
& \mathcal{T}_{\varepsilon}^{\mathcal{S}}\left(\phi_{\varepsilon}\right) \mathbf{1}_{\widehat{\Omega}_{\varepsilon}^{i n t} \times \mathcal{S}} \longrightarrow \Phi \quad \text { strongly in } L^{2}\left(\Omega ; H^{1}(\mathcal{S})\right), \\
& \mathcal{T}_{\varepsilon}^{\mathcal{S}}\left(\frac{d \phi_{\varepsilon}}{d \mathbf{s}}\right) \mathbf{1}_{\widehat{\Omega}_{\varepsilon}^{i n t} \times \mathcal{S}} \rightarrow \nabla \Phi \cdot \mathbf{t}_{1}+\frac{\partial \widehat{\phi}}{\partial \mathbf{S}} \quad \text { weakly in } L^{2}(\Omega \times \mathcal{S}) .
\end{aligned}
$$


Proof The proof is postponed in Appendix C.

Denote

$$
H_{\Gamma}^{1}(\Omega) \doteq\left\{\phi \in H^{1}(\Omega) \mid \phi=0 \text { on } \Gamma\right\}
$$

Corollary 2 Let $\left\{\phi_{\varepsilon}\right\}_{\varepsilon}$ be a sequence offunctions belonging to $H^{1}\left(\mathcal{S}_{\varepsilon}\right)^{3} \cap \mathbf{V}_{\varepsilon, r}$ and satisfying the following

$$
\left\|\phi_{\varepsilon}\right\|_{H^{1}\left(\mathcal{S}_{\varepsilon}\right)} \leq \frac{C}{\varepsilon} .
$$

Then, up to a subsequence, there exists $(\Phi, \widehat{\phi}) \in H_{\Gamma}^{1}(\Omega)^{3} \times L^{2}\left(\Omega ; H_{p e r}^{1}(\mathcal{S})\right)^{3}$ such that

$$
\begin{aligned}
& \mathcal{T}_{\varepsilon}^{\mathcal{S}}\left(\phi_{\varepsilon}\right) \mathbf{1}_{\widehat{\Omega}_{\varepsilon}^{\prime i n t} \times \mathcal{S}} \longrightarrow \Phi \quad \text { strongly in } L^{2}\left(\Omega ; H^{1}(\mathcal{S})\right)^{3}, \\
& \mathcal{T}_{\varepsilon}^{\mathcal{S}}\left(\frac{d \phi_{\varepsilon}}{d \mathbf{s}}\right) \mathbf{1}_{\widehat{\Omega}_{\varepsilon}^{\prime i n t} \times \mathcal{S}} \rightarrow \nabla \Phi \cdot \mathbf{t}_{1}+\frac{\partial \widehat{\phi}}{\partial \mathbf{S}} \quad \text { weakly in } L^{2}(\Omega \times \mathcal{S})^{3} .
\end{aligned}
$$

Proof Since $\left\{\phi_{\varepsilon}\right\}_{\varepsilon}$ belongs to $V_{\varepsilon, r}$, these functions equal to 0 in $\mathcal{S}_{\varepsilon}^{\prime} \backslash \mathcal{S}_{\varepsilon}$. Applying Lemma 15 with $\mathcal{S}_{\varepsilon}^{\prime}$ instead $\mathcal{S}_{\varepsilon}$ and with $\Omega^{\prime}$ instead $\Omega$ give the result.

\section{Asymptotic Behaviors}

\subsection{Asymptotic Behavior of a Sequence of Displacements}

From now on, we assume that $r$ is a function of $\varepsilon$ satisfying the following conditions:

$$
\lim _{\varepsilon \rightarrow 0} \frac{r}{\varepsilon}=0, \quad \lim _{\varepsilon \rightarrow 0} \frac{r}{\varepsilon^{2}}=\kappa \in[0,+\infty] .
$$

In addition, every field appearing in the decomposition introduced in the previous sections will be denoted with only the index $\varepsilon$.

In this section we consider a sequence $\left\{u_{\varepsilon}\right\}_{\varepsilon}$ of displacements belonging to $\mathbf{V}_{\varepsilon, r}$ and satisfying

$$
\left\|e\left(u_{\varepsilon}\right)\right\|_{L^{2}\left(\mathcal{S}_{\varepsilon, r}\right)} \leq C .
$$

Theorem 1 For a subsequence of $\{\varepsilon\}$, still denoted $\{\varepsilon\}$, one has

(i) there exist $\mathcal{U} \in H_{\Gamma}^{1}(\Omega)^{3}, \overline{\mathcal{U}} \in L^{2}\left(\Omega ; H_{\text {per }}^{1}(\mathcal{S})\right)^{3}$ such that $S \longmapsto \overline{\mathcal{U}}(\cdot, S) \wedge \mathbf{t}_{1}$ is an affine function on every segment of $\mathcal{S}$ and the following convergences hold:

$$
\begin{aligned}
& \frac{r}{\varepsilon} \mathcal{U}_{\varepsilon} \mathbf{1}_{\Omega_{\varepsilon} \text { int }} \rightarrow \mathcal{U} \quad \text { weakly in } L^{2}(\Omega)^{3}, \\
& \frac{r}{\varepsilon} \nabla \mathcal{U}_{\varepsilon} \mathbf{1}_{\Omega_{\varepsilon}^{i n t}} \rightarrow \nabla \mathcal{U} \quad \text { weakly in } L^{2}(\Omega)^{9}, \\
& \frac{r}{\varepsilon} \mathcal{T}_{\varepsilon}^{\mathcal{S}}\left(\mathcal{U}_{\varepsilon}^{h}+\left(\overline{\mathcal{U}}_{\varepsilon} \cdot \mathbf{t}_{1}\right) \mathbf{t}_{1}\right) \rightarrow \mathcal{U} \quad \text { weakly in } L^{2}\left(\Omega ; H^{1}(\mathcal{S})\right)^{3}, \\
& \frac{r}{\varepsilon} \mathcal{T}_{\varepsilon}^{\mathcal{S}}\left(\frac{d}{d \mathbf{s}}\left(\mathcal{U}_{\varepsilon}^{h}+\left(\overline{\mathcal{U}}_{\varepsilon} \cdot \mathbf{t}_{1}\right) \mathbf{t}_{1}\right)\right) \rightarrow \nabla \mathcal{U} \mathbf{t}_{1}+\frac{\partial \overline{\mathcal{U}}}{\partial \mathbf{S}} \quad \text { weakly in } L^{2}(\Omega \times \mathcal{S})^{3}, \\
& \frac{r}{\varepsilon} \mathcal{T}_{\varepsilon}^{\mathcal{S}}\left(\frac{d \mathcal{U}_{\varepsilon}}{d \mathbf{s}}\right) \cdot \mathbf{t}_{1} \rightarrow\left(e(\mathcal{U}) \mathbf{t}_{1}\right) \cdot \mathbf{t}_{1}+\frac{\partial \overline{\mathcal{U}}}{\partial \mathbf{S}} \cdot \mathbf{t}_{1} \quad \text { weakly in } L^{2}(\Omega \times \mathcal{S}),
\end{aligned}
$$


where $e(\mathcal{U})$ is the symmetric gradient of the displacement $\mathcal{U}$

(ii) there exists $\widehat{\mathcal{U}} \in L^{2}\left(\Omega ; H_{\text {per }}^{1}(\mathcal{S})\right)^{3}$ such that $\widehat{\mathcal{U}}_{\mid \gamma_{\ell}} \in L^{2}\left(\Omega ; H_{0}^{1}\left(\gamma_{\ell}\right) \cap H^{2}\left(\gamma_{\ell}\right)\right)^{3}, \widehat{\mathcal{U}}_{\mid \gamma_{\ell}}$. $\mathbf{t}_{1}^{\ell}=0, \ell \in\{1, \ldots, m\}$ and

$$
\frac{r^{2}}{\varepsilon^{3}} \mathcal{T}_{\varepsilon}^{\mathcal{S}}\left(\overline{\mathcal{U}}_{\varepsilon}-\left(\overline{\mathcal{U}}_{\varepsilon} \cdot \mathbf{t}_{1}\right) \mathbf{t}_{1}\right) \rightarrow \widehat{\mathcal{U}} \quad \text { weakly in } \quad L^{2}\left(\Omega ; H^{1}(\mathcal{S})\right)^{3},
$$

(iii) there exists $\mathcal{Z} \in L^{2}(\Omega \times \mathcal{S})^{3}$ such that

$$
\frac{r}{\varepsilon} \mathcal{T}_{\varepsilon}^{\mathcal{S}}\left(\frac{d \mathcal{U}_{\varepsilon}}{d \mathbf{s}}-\mathcal{R}_{\varepsilon} \wedge \mathbf{t}_{1}\right) \rightarrow \nabla \mathcal{U} \mathbf{t}_{1}+\frac{\partial \overline{\mathcal{U}}}{\partial \mathbf{S}}+\mathcal{Z} \quad \text { weakly in } L^{2}(\Omega \times \mathcal{S})^{3},
$$

(iv) there exists $\widehat{\mathcal{R}} \in L^{2}\left(\Omega ; H_{\text {per }}^{1}(\mathcal{S})\right)^{3}$ such that

$$
\frac{r^{2}}{\varepsilon^{2}} \mathcal{T}_{\varepsilon}^{\mathcal{S}}\left(\mathcal{R}_{\varepsilon}\right) \rightarrow \widehat{\mathcal{R}} \quad \text { weakly in } \quad L^{2}\left(\Omega ; H^{1}(\mathcal{S})\right)^{3}
$$

and

$$
\widehat{\mathcal{R}} \wedge \mathbf{t}_{1}=\frac{\partial \widehat{\mathcal{U}}}{\partial \mathbf{S}},
$$

(v) there exists $\bar{u} \in L^{2}\left(\Omega \times \mathcal{S} ; H^{1}(D)\right)^{3}$ such that

$$
\begin{aligned}
& \frac{1}{\varepsilon} \mathcal{T}_{\varepsilon}^{b, \ell}\left(\bar{u}_{\varepsilon}\right) \rightarrow \bar{u} \quad \text { weaklyin } L^{2}\left(\Omega \times \gamma_{\ell} ; H^{1}(D)\right)^{3}, \\
& \frac{r}{\varepsilon^{2}} \frac{\partial}{\partial S_{1}} \mathcal{T}_{\varepsilon}^{b, \ell}\left(\bar{u}_{\varepsilon}\right) \rightarrow 0 \quad \text { weakly in } L^{2}\left(\Omega \times \gamma_{\ell} \times D\right)^{3} .
\end{aligned}
$$

Proof Below, every convergence is up to a subsequence of $\{\varepsilon\}$ still denoted $\{\varepsilon\}$.

(i) From Lemma 21 and Proposition 3 we have the following estimates:

$$
\frac{r}{\varepsilon}\left\|\mathcal{U}_{\varepsilon}\right\|_{H^{1}\left(\Omega_{\varepsilon}^{\prime i n t}\right)} \leq C
$$

Lemma 5.1 in [16] gives a field $\mathcal{U} \in H_{\Gamma}^{1}(\Omega)^{3}$ such that (7.2) $)_{1,2}$ hold.

From the estimates (5.10) and (A.2) one obtains

$$
\left\|\mathcal{U}_{\varepsilon}^{h}+\left(\overline{\mathcal{U}}_{\varepsilon} \cdot \mathbf{t}_{1}\right) \mathbf{t}_{1}\right\|_{H^{1}\left(\mathcal{S}_{\varepsilon}\right)} \leq \frac{C}{r} .
$$

Hence, the convergences (7.2) $)_{3,4}$ are the consequences of Corollary 2.

Since

$$
\frac{d \mathcal{U}_{\varepsilon}}{d \mathbf{s}} \cdot \mathbf{t}_{1}=\frac{d}{d \mathbf{s}}\left(\mathcal{U}_{\varepsilon}^{h}+\left(\overline{\mathcal{U}}_{\varepsilon} \cdot \mathbf{t}_{1}\right) \mathbf{t}_{1}\right) \cdot \mathbf{t}_{1},
$$

the convergence $(7.2)_{5}$ holds (observe that $\left.\left(\nabla \mathcal{U} \mathbf{t}_{1}\right) \cdot \mathbf{t}_{1}=\left(e(\mathcal{U}) \mathbf{t}_{1}\right) \cdot \mathbf{t}_{1}\right)$.

(ii) From $(5.10),(5.22),(1)$ and the fact that by construction $\overline{\mathcal{U}}_{\varepsilon \mid \gamma_{\ell}}(0)=\overline{\mathcal{U}}_{\varepsilon \mid \gamma_{\ell}}\left(\varepsilon l_{\ell}\right)=0$, we obtain

$$
\frac{r^{2}}{\varepsilon^{3}}\left\|\mathcal{T}_{\varepsilon}^{\mathcal{S}}\left(\overline{\mathcal{U}}_{\varepsilon}-\left(\overline{\mathcal{U}}_{\varepsilon} \cdot \mathbf{t}_{1}\right) \mathbf{t}_{1}\right)\right\|_{L^{2}\left(\Omega ; H_{0}^{1} \gamma_{\ell}\right)} \leq C
$$

Thus, up to a subsequence, there exists $\widehat{\mathcal{U}} \in L^{2}\left(\Omega ; H^{1}(\mathcal{S})\right)^{3}$ such that $\widehat{\mathcal{U}}_{\mid \gamma_{\ell}} \in L^{2}\left(\Omega ; H_{0}^{1}\left(\gamma_{\ell}\right)\right)^{3}$, $\widehat{\mathcal{U}}_{\mid \gamma \ell} \cdot \mathbf{t}_{1}^{\ell}=0, \ell \in\{1, \ldots, m\}$ and convergence $(7.3)_{1}$ holds. 
(iii) Estimates (5.9) 4 -(5.10) and (6.2) yield

$$
\left\|\mathcal{T}_{\varepsilon}^{\mathcal{S}}\left(\frac{d}{d \mathbf{s}}\left(\overline{\mathcal{U}}_{\varepsilon}-\left(\overline{\mathcal{U}}_{\varepsilon} \cdot \mathbf{t}_{1}\right) \mathbf{t}_{1}\right)-\mathcal{R}_{\varepsilon} \wedge \mathbf{t}_{1}\right)\right\|_{L^{2}(\Omega \times \mathcal{S})} \leq C \frac{\varepsilon}{r} .
$$

Then, there exists a field $\mathcal{Z} \in L^{2}(\Omega \times \mathcal{S})^{3}$ such that

$$
{ }_{\varepsilon}^{r} \mathcal{T}_{\varepsilon}^{\mathcal{S}}\left(\frac{d}{d \mathbf{s}}\left(\overline{\mathcal{U}}_{\varepsilon}-\left(\overline{\mathcal{U}}_{\varepsilon} \cdot \mathbf{t}_{1}\right) \mathbf{t}_{1}\right)-\mathcal{R}_{\varepsilon} \wedge \mathbf{t}_{1}\right) \rightarrow \mathcal{Z} \quad \text { weakly in } \quad L^{2}(\Omega \times \mathcal{S})^{3}
$$

and by $(7.2)_{4}$ we have

$$
\begin{aligned}
{ }_{\frac{r}{\varepsilon}} \mathcal{T}_{\varepsilon}^{\mathcal{S}}\left(\frac{d \mathcal{U}_{\varepsilon}}{d \mathbf{s}}-\mathcal{R}_{\varepsilon} \wedge \mathbf{t}_{1}\right)= & \frac{r}{\varepsilon} \mathcal{T}_{\varepsilon}^{\mathcal{S}}\left(\frac{d}{d \mathbf{s}}\left(\mathcal{U}_{\varepsilon}^{h}+\left(\overline{\mathcal{U}}_{\varepsilon} \cdot \mathbf{t}_{1}\right) \mathbf{t}_{1}\right)\right) \\
& +\frac{r}{\varepsilon} \mathcal{T}_{\varepsilon}^{\mathcal{S}}\left(\frac{d}{d \mathbf{s}}\left(\overline{\mathcal{U}}_{\varepsilon}-\left(\overline{\mathcal{U}}_{\varepsilon} \cdot \mathbf{t}_{1}\right) \mathbf{t}_{1}\right)-\mathcal{R}_{\varepsilon} \wedge \mathbf{t}_{1}\right) \\
& \rightarrow \nabla \mathcal{U} \mathbf{t}_{1}+\frac{\partial \overline{\mathcal{U}}}{\partial \mathbf{S}}+\mathcal{Z} \quad \text { weakly in } \quad L^{2}(\Omega \times \mathcal{S})^{3}
\end{aligned}
$$

(iv) Estimate $(5.18)_{2}$ gives

$$
\left\|\mathcal{R}_{\varepsilon}\right\|_{L^{2}\left(\mathcal{S}_{\varepsilon}\right)}+\varepsilon\left\|\frac{d \mathcal{R}_{\varepsilon}}{d \mathbf{s}}\right\|_{L^{2}\left(\mathcal{S}_{\varepsilon}\right)} \leq C \frac{\varepsilon}{r^{2}}
$$

Thus, up to a subsequence, there exists a function $\widehat{\mathcal{R}} \in L^{2}\left(\Omega ; H_{p e r}^{1}(\mathcal{S})\right)^{3}$ (see Lemma 14) such that (7.5) holds.

On the one hand, from (7.9) we have

$$
\frac{r^{2}}{\varepsilon^{2}} \mathcal{T}_{\varepsilon}^{\mathcal{S}}\left(\frac{d}{d \mathbf{s}}\left(\overline{\mathcal{U}}_{\varepsilon}-\left(\overline{\mathcal{U}}_{\varepsilon} \cdot \mathbf{t}_{1}\right) \mathbf{t}_{1}\right)-\mathcal{R}_{\varepsilon} \wedge \mathbf{t}_{1}\right) \longrightarrow 0 \quad \text { strongly in } \quad L^{2}(\Omega \times \mathcal{S})^{3}
$$

On the other hand from convergences $(7.3)_{1},(7.5)$ we obtain

$$
\frac{r^{2}}{\varepsilon^{2}} \mathcal{T}_{\varepsilon}^{\mathcal{S}}\left(\frac{d}{d \mathbf{s}}\left(\overline{\mathcal{U}}_{\varepsilon}-\left(\overline{\mathcal{U}}_{\varepsilon} \cdot \mathbf{t}_{1}\right) \mathbf{t}_{1}\right)-\mathcal{R}_{\varepsilon} \wedge \mathbf{t}_{1}\right) \rightarrow \frac{\partial \widehat{\mathcal{U}}}{d \mathbf{S}}-\widehat{\mathcal{R}} \wedge \mathbf{t}_{1} \quad \text { weakly in } \quad L^{2}(\Omega \times \mathcal{S})^{3} .
$$

Hence, we obtain (7.6) and

$$
\frac{\partial \widehat{\mathcal{R}}}{\partial \mathbf{S}} \wedge \mathbf{t}_{1}=\frac{\partial^{2} \widehat{\mathcal{U}}}{\partial \mathbf{S}^{2}} \text { a.e. in } \Omega \times \mathcal{S}
$$

Then $\widehat{\mathcal{U}}_{\mid \gamma_{\ell}} \in L^{2}\left(\Omega ; H_{0}^{1}\left(\gamma_{\ell}\right) \cap H^{2}\left(\gamma_{\ell}\right)\right)^{3}$.

(v) Taking into account $(5.9)_{1,2},(6.7)_{2}$ and (6.5) for $j=2,3, \ell \in\{1, \ldots, m\}$, we have

$$
\left\|\mathcal{T}_{\varepsilon}^{b, \ell}\left(\bar{u}_{\varepsilon}\right)\right\|_{L^{2}\left(\Omega \times \gamma_{\ell} \times D\right)}+\left\|\frac{\partial}{\partial S_{j}} \mathcal{T}_{\varepsilon}^{b, \ell}\left(\bar{u}_{\varepsilon}\right)\right\|_{L^{2}\left(\Omega \times \gamma_{\ell} \times D\right)} \leq C \varepsilon .
$$

Hence, up to a subsequence, there exists $\bar{u} \in L^{2}\left(\Omega \times \mathcal{S} ; H^{1}(D)\right)^{3}$ such that $(7.7)_{1}$ holds. In order to show convergence $(7.7)_{2}$, note that from $(5.9)_{2},(6.7)_{1}$ and (6.5) it follows

$$
\frac{r}{\varepsilon^{2}}\left\|\frac{\partial}{\partial S_{1}} \mathcal{T}_{\varepsilon}^{b, \ell}\left(\bar{u}_{\varepsilon}\right)\right\|_{L^{2}\left(\Omega \times \gamma_{\ell} \times D\right)} \leq C .
$$


Therefore, convergence $(7.7)_{2}$ is proved, since

$$
\frac{r}{\varepsilon^{2}} \mathcal{T}_{\varepsilon}^{b, \ell}\left(\bar{u}_{\varepsilon}\right) \longrightarrow 0 \quad \text { strongly in } \quad L^{2}\left(\Omega \times \gamma_{\ell} ; H^{1}(D)\right)^{3} .
$$

Remark 3 Due to (4.2), the warping $\bar{u}$ satisfies

$$
\begin{aligned}
& \int_{D} \bar{u}\left(\cdot, S_{2}, S_{3}\right) d S_{2} d S_{3}=0, \\
& \int_{D} \bar{u}\left(\cdot, S_{2}, S_{3}\right) \wedge\left(S_{2} \mathbf{t}_{2}^{\ell}+S_{3} \mathbf{t}_{3}^{\ell}\right) d S_{2} d S_{3}=0,
\end{aligned}
$$

Denote

$\mathcal{D}_{E x} \doteq\left\{\overline{\mathcal{A}} \in H_{p e r, 0}^{1}(\mathcal{S})^{3} \mid \overline{\mathcal{A}} \wedge \mathbf{t}_{1}\right.$ is an affine function on every segment $\left.\gamma_{\ell}, \ell \in\{1, \ldots, m\}\right\}$, $\mathcal{D}_{\text {In }} \doteq\left\{(\widehat{\mathcal{A}}, \widehat{\mathcal{B}}) \in H_{\text {per }}^{1}(\mathcal{S})^{3} \times H_{\text {per }}^{1}(\mathcal{S})^{3} \mid \frac{d \widehat{\mathcal{A}}}{d \mathbf{S}}=\widehat{\mathcal{B}} \wedge \mathbf{t}_{1}, \quad \widehat{\mathcal{A}}=0\right.$ on all the nodes of $\left.\mathcal{S}\right\}$.

The field $\overline{\mathcal{U}}$ is in $L^{2}\left(\Omega ; \mathcal{D}_{E x}\right)$ while the pair $(\widehat{\mathcal{U}}, \widehat{\mathcal{R}})$ belongs to $L^{2}\left(\Omega ; \mathcal{D}_{\text {In }}\right)$. It worth to notice that a field $\overline{\mathcal{A}}$ belonging to $H_{\text {per }, 0}^{1}(\mathcal{S})^{3}$ is a local extensional displacement if and only if

$$
\int_{\mathcal{S}} \frac{d \overline{\mathcal{A}}}{d \mathbf{S}} \cdot \frac{d \widehat{\mathcal{A}}}{d \mathbf{S}} d \mathbf{S}=0
$$

for all $\widehat{\mathcal{A}} \in H_{\text {per }}^{1}(\mathcal{S})^{3}$ which is the first component of an element belonging to $\mathcal{D}_{\text {In }}$.

We endow $\mathcal{D}_{E x}\left(\right.$ resp. $\left.\mathcal{D}_{I n}\right)$ with the semi-norm

$$
\|\overline{\mathcal{A}}\|_{\mathcal{S}} \doteq\left\|\frac{d \overline{\mathcal{A}}}{d \mathbf{S}} \cdot \mathbf{t}_{1}\right\|_{L^{2}(\mathcal{S})}, \quad\left(\operatorname{resp} . \quad\|(\widehat{\mathcal{A}}, \widehat{\mathcal{B}})\|_{\mathcal{D}_{I n}} \doteq\left\|\frac{d \widehat{\mathcal{B}}}{d \mathbf{S}}\right\|_{L^{2}(\mathcal{S})}\right) .
$$

Lemma 16 On $\mathcal{D}_{E x}$ the semi-norm $\|\cdot\|_{\mathcal{S}}$ is a norm equivalent to the norm of $H^{1}(\mathcal{S})^{3}$. On $\mathcal{D}_{\text {In }}$ the semi-norm $\|(\cdot, \cdot)\|_{\mathcal{D}_{\text {In }}}$ is a norm equivalent to the norm of $H^{1}(\mathcal{S})^{3} \times H^{1}(\mathcal{S})^{3}$.

Proof The proof is given in Appendix D.

\subsection{Asymptotic Behavior of the Strain Tensor}

For every $\mathcal{V} \in H_{\Gamma}^{1}(\Omega)^{3},(\overline{\mathcal{V}}, \widehat{\mathcal{V}}, \widehat{\mathcal{B}}) \in L^{2}\left(\Omega ; \mathcal{D}_{E x} \times \mathcal{D}_{I n}\right)$ and $\widetilde{v} \in L^{2}\left(\Omega \times \mathcal{S} ; H^{1}(D)\right)^{3}$ we define the symmetric tensors $\mathcal{E}, \mathcal{E}_{\mathcal{S}}, \mathcal{E}_{D}$ by

$$
\begin{aligned}
& \mathcal{E}(\mathcal{V}) \doteq\left(\begin{array}{ccc}
\left(e(\mathcal{V}) \mathbf{t}_{1}\right) \cdot \mathbf{t}_{1} & 0 & 0 \\
0 & 0 & 0 \\
0 & 0 & 0
\end{array}\right), \\
& \mathcal{E}_{S}(\overline{\mathcal{V}}, \widehat{\mathcal{V}}, \widehat{\mathcal{B}}) \doteq\left(\begin{array}{ccc}
\frac{\partial \overline{\mathcal{V}}}{\partial S_{1}} \cdot \mathbf{t}_{1}-\frac{\partial^{2} \widehat{\mathcal{V}}}{\partial S_{1}^{2}} \cdot\left(S_{2} \mathbf{t}_{2}+S_{3} \mathbf{t}_{3}\right) & * & * \\
-\frac{S_{3}}{2} \frac{\partial \mathcal{\mathcal { B }}}{\partial S_{1}} \cdot \mathbf{t}_{1} & 0 & 0 \\
\frac{S_{2}}{2} \frac{\partial \widehat{\mathcal{B}}}{\partial S_{1}} \cdot \mathbf{t}_{1} & 0 & 0
\end{array}\right),
\end{aligned}
$$




$$
\mathcal{E}_{D}(\widetilde{v}) \doteq\left(\begin{array}{ccc}
0 & \frac{1}{2} \frac{\partial \widetilde{v}}{\partial S_{2}} \cdot \mathbf{t}_{1} & \frac{1}{2} \frac{\partial \widetilde{v}}{\partial S_{3}} \cdot \mathbf{t}_{1} \\
* & \frac{\partial \widetilde{v}}{\partial S_{2}} \cdot \mathbf{t}_{2} & \frac{1}{2} \frac{\partial \widetilde{v}}{\partial S_{3}} \cdot \mathbf{t}_{2}+\frac{1}{2} \frac{\partial \widetilde{v}}{\partial S_{2}} \cdot \mathbf{t}_{3} \\
* & * & \frac{\partial \widetilde{v}}{\partial S_{3}} \cdot \mathbf{t}_{3}
\end{array}\right) \quad \text { a.e. in } \Omega \times \mathcal{S} \times D
$$

Theorem 2 Let $u_{\varepsilon}$ be the solution to (5.8). There exist a subsequence of $\{\varepsilon\}$, still denoted $\{\varepsilon\}$, and $\mathcal{U} \in H_{\Gamma}^{1}(\Omega)^{3},(\overline{\mathcal{U}}, \widehat{\mathcal{U}}, \widehat{\mathcal{R}}) \in L^{2}\left(\Omega ; \mathcal{D}_{E x} \times \mathcal{D}_{\text {In }}\right)$ and $\tilde{u} \in L^{2}\left(\Omega \times \mathcal{S} ; H^{1}(D)\right)^{3}$ such that the following convergences hold $(\ell \in\{1, \ldots, m\})$ :

$$
\begin{cases}\frac{r}{\varepsilon} \mathcal{T}_{\varepsilon}^{b, \ell}\left(u_{\varepsilon}\right) \rightarrow \mathcal{U}+\frac{1}{\kappa} \widehat{\mathcal{U}} \quad \text { weakly in } L^{2}\left(\Omega \times \gamma_{\ell} ; H^{1}(D)\right)^{3}, & \text { if } \kappa \in(0,+\infty], \\ \frac{r^{2}}{\varepsilon^{3}} \mathcal{T}_{\varepsilon}^{b, \ell}\left(u_{\varepsilon}\right) \rightarrow \widehat{\mathcal{U}} \quad \text { weakly in } L^{2}\left(\Omega \times \gamma_{\ell} ; H^{1}(D)\right)^{3}, & \text { if } \kappa=0\end{cases}
$$

and

$$
{ }_{\bar{\varepsilon}}^{r} \mathcal{T}_{\varepsilon}^{b, \ell}\left(e_{s}\left(u_{\varepsilon}\right)\right) \rightarrow \mathcal{E}(\mathcal{U})+\mathcal{E}_{S}(\overline{\mathcal{U}}, \widehat{\mathcal{U}}, \widehat{\mathcal{R}})+\mathcal{E}_{D}(\widetilde{u}) \quad \text { weakly in } \quad L^{2}\left(\Omega \times \gamma_{\ell} \times D\right)^{3 \times 3}
$$

Proof Below, we give the asymptotic behavior of the sequence $\left\{\mathcal{T}_{\varepsilon}^{b, \ell}\left(u_{\varepsilon}\right)\right\}$ as $\varepsilon \rightarrow 0$ and $r / \varepsilon \rightarrow 0$. One has

$$
\mathcal{T}_{\varepsilon}^{b, \ell}\left(u_{\varepsilon}\right)=\mathcal{T}_{\varepsilon}^{b, \ell}\left(U_{\varepsilon}^{e}\right)+\mathcal{T}_{\varepsilon}^{b, \ell}\left(\bar{u}_{\varepsilon}\right) .
$$

From $(7.7)_{1}$ we have $(\ell \in\{1, \ldots, m\})$

$$
\frac{1}{\varepsilon} \mathcal{T}_{\varepsilon}^{b, \ell}\left(\bar{u}_{\varepsilon}\right) \rightarrow \bar{u} \quad \text { weakly in } \quad L^{2}\left(\Omega \times \gamma_{\ell} ; H^{1}(D)\right)^{3} .
$$

From Definition 3 we have $(\ell \in\{1, \ldots, m\})$

$$
\begin{aligned}
& \mathcal{T}_{\varepsilon}^{b, \ell}\left(U_{\varepsilon}^{e}\right)=\mathcal{T}_{\varepsilon}^{\mathcal{S}}\left(\mathcal{U}_{\varepsilon}^{h}+\left(\overline{\mathcal{U}}_{\varepsilon} \cdot \mathbf{t}_{1}\right) \mathbf{t}_{1}\right)+\mathcal{T}_{\varepsilon}^{\mathcal{S}}\left(\overline{\mathcal{U}}_{\varepsilon}-\left(\overline{\mathcal{U}}_{\varepsilon} \cdot \mathbf{t}_{1}\right) \mathbf{t}_{1}\right)+r \mathcal{T}_{\varepsilon}^{\mathcal{S}}\left(\mathcal{R}_{\varepsilon}\right) \wedge\left(S_{2} \mathbf{t}_{2}^{\ell}+S_{3} \mathbf{t}_{3}^{\ell}\right), \\
& \text { a.e. in } \Omega \times \gamma_{\ell} \times D .
\end{aligned}
$$

The convergences $(7.2)_{3},(7.3),(7.5)$ yield

$$
\frac{r}{\varepsilon} \mathcal{T}_{\varepsilon}^{b, \ell}\left(U_{\varepsilon}^{e}\right) \longrightarrow \mathcal{U}+\frac{1}{\kappa} \widehat{\mathcal{U}} \quad \text { weakly in } L^{2}\left(\Omega \times \gamma_{\ell} ; H^{1}(D)\right)^{3}, \quad \text { if } \kappa \in(0,+\infty],
$$

if $\kappa=0$, from (7.3) we obtain

$$
\frac{r^{2}}{\varepsilon^{3}} \mathcal{T}_{\varepsilon}^{b, \ell}\left(U_{\varepsilon}^{e}\right) \rightarrow \widehat{\mathcal{U}} \quad \text { weakly in } L^{2}\left(\Omega \times \gamma_{\ell} ; H^{1}(D)\right)^{3} .
$$

Hence, the convergences (7.12) hold.

Now we consider the asymptotic behavior of the strain tensors $\mathcal{T}_{\varepsilon}^{b, \ell}\left(e_{s}\left(u_{\varepsilon}\right)\right)$

$$
\mathcal{T}_{\varepsilon}^{b, \ell}\left(e_{s}\left(u_{\varepsilon}\right)\right)=\mathcal{T}_{\varepsilon}^{b, \ell}\left(e_{s}\left(\bar{u}_{\varepsilon}\right)\right)+\mathcal{T}_{\varepsilon}^{b, \ell}\left(e_{s}\left(U_{e}^{e}\right)\right) .
$$

From (7.7), we obtain $(\ell \in[1, \ldots, m])$

$$
{ }_{\varepsilon}^{r} \mathcal{T}_{\varepsilon}^{b, \ell}\left(e_{s}\left(\bar{u}_{\varepsilon}\right)\right) \rightarrow \mathcal{E}_{D}(\bar{u}) \quad \text { weakly in } \quad L^{2}\left(\Omega \times \gamma_{\ell} \times D\right)^{3 \times 3} .
$$


Next from the convergences $(7.2)_{4},(7.3)_{2},(7.5)$ and (7.6) we obtain

$$
\frac{r}{\varepsilon} \mathcal{T}_{\varepsilon}^{b, \ell}\left(e_{s}\left(U_{\varepsilon}^{e}\right)\right) \rightarrow\left(\begin{array}{ccc}
\left(\nabla \mathcal{U} \mathbf{t}_{1}^{\ell}+\frac{\partial \overline{\mathcal{U}}}{\partial S_{1}}\right) \cdot \mathbf{t}_{1}^{\ell}-\frac{\partial^{2} \widehat{\mathcal{U}}}{\partial S_{1}^{2}} \cdot\left(S_{2} \cdot \mathbf{t}_{2}^{\ell}+S_{3} \cdot \mathbf{t}_{3}^{\ell}\right) & * & * \\
\frac{1}{2}\left(\nabla \mathcal{U} \mathbf{t}_{1}+\frac{\partial \overline{\mathcal{U}}}{\partial S_{1}}+\mathcal{Z}\right) \cdot \mathbf{t}_{2}^{\ell}-\frac{S_{3}}{2} \frac{\partial \widehat{\mathcal{R}}}{\partial S_{1}} \cdot \mathbf{t}_{1}^{\ell} & 0 & 0 \\
\frac{1}{2}\left(\nabla \mathcal{U} \mathbf{t}_{1}+\frac{\partial \overline{\mathcal{U}}}{\partial S_{1}}+\mathcal{Z}\right) \cdot \mathbf{t}_{3}^{\ell}+\frac{S_{2}}{2} \frac{\partial \widehat{\mathcal{R}}}{\partial S_{1}} \cdot \mathbf{t}_{1}^{\ell} & 0 & 0
\end{array}\right)
$$

weakly in $L^{2}\left(\Omega \times \gamma_{\ell} \times D\right)^{3 \times 3}$.

We set

$\tilde{u}=\bar{u}+S_{2}\left(\left(\nabla \mathcal{U} \mathbf{t}_{1}+\frac{\partial \overline{\mathcal{U}}}{\partial S_{1}}+\mathcal{Z}\right) \cdot \mathbf{t}_{2}\right) \mathbf{t}_{1}+S_{3}\left(\left(\nabla \mathcal{U} \mathbf{t}_{1}+\frac{\partial \overline{\mathcal{U}}}{\partial S_{1}}+\mathcal{Z}\right) \cdot \mathbf{t}_{3}\right) \mathbf{t}_{1} \quad$ a.e. in $\Omega \times \mathcal{S} \times D$

Hence, one has

$$
\frac{r}{\varepsilon} \mathcal{T}_{\varepsilon}^{b, \ell}\left(e_{s}\left(u_{\varepsilon}\right)\right) \rightarrow \mathcal{E}(\mathcal{U})+\mathcal{E}_{S}(\overline{\mathcal{U}}, \widehat{\mathcal{U}}, \widehat{\mathcal{R}})+\mathcal{E}_{D}(\widetilde{\mathcal{u}}) \quad \text { weakly in } \quad L^{2}\left(\Omega \times \gamma_{\ell} \times D\right)^{3 \times 3}
$$

and (7.13) holds.

Denote

$$
\begin{aligned}
\mathcal{D}_{w}=\left\{\left(\widetilde{w}_{1}, \widetilde{w}_{2}, \widetilde{w}_{3}\right) \in H^{1}(D)^{3} \mid\right. & \int_{D}\left(S_{3} \widetilde{w}_{2}\left(S_{2}, S_{3}\right)-S_{2} \widetilde{w}_{3}\left(S_{2}, S_{3}\right)\right) d S_{2} d S_{3}=0, \\
& \left.\int_{D} \widetilde{w}_{i}\left(S_{2}, S_{3}\right) d S_{2} d S_{3}=0, i \in\{1,2,3\}\right\} .
\end{aligned}
$$

Thanks to the conditions (7.11) satisfied by $\bar{u}$ and the definition of $\tilde{u}$, one obtains

$$
\tilde{u}=\left(\tilde{u} \cdot \mathbf{t}_{1}\right) \mathbf{t}_{1}+\left(\tilde{u} \cdot \mathbf{t}_{2}\right) \mathbf{t}_{2}+\left(\tilde{u} \cdot \mathbf{t}_{3}\right) \mathbf{t}_{3} \text { is such that }\left(\tilde{u} \cdot \mathbf{t}_{1}, \tilde{u} \cdot \mathbf{t}_{2}, \tilde{u} \cdot \mathbf{t}_{3}\right) \in L^{2}\left(\Omega \times \mathcal{S} ; \mathcal{D}_{w}\right) \text {. }
$$

For the sake of simplicity, if $\widetilde{v}$ belongs to $L^{2}\left(\Omega \times \mathcal{S} ; H^{1}(D)^{3}\right)$ and is such that

$$
\widetilde{v}=\left(\widetilde{v} \cdot \mathbf{t}_{1}\right) \mathbf{t}_{1}+\left(\widetilde{v} \cdot \mathbf{t}_{2}\right) \mathbf{t}_{2}+\left(\widetilde{v} \cdot \mathbf{t}_{3}\right) \mathbf{t}_{3} \text { satisfies }\left(\tilde{v} \cdot \mathbf{t}_{1}, \widetilde{v} \cdot \mathbf{t}_{2}, \widetilde{v} \cdot \mathbf{t}_{3}\right) \in L^{2}\left(\Omega \times \mathcal{S} ; \mathcal{D}_{w}\right)
$$

we will write that $\tilde{v}$ belongs to $L^{2}\left(\Omega \times \mathcal{S} ; \mathcal{D}_{w}\right)$.

\section{The Limit Unfolded Problem}

To obtain the limit unfolded problem, we will choose test displacements $v$ in $\mathbf{V}_{\varepsilon, r}$ which vanish in the junction domain $\mathcal{J}_{\varepsilon, r}$ or which are equal to rigid displacements in $\mathcal{J}_{\varepsilon, r}$. In doing so, we will have

$$
\int_{\mathcal{S}_{\varepsilon, r}} \sigma\left(u_{\varepsilon}\right): e(v) d x=\sum_{\ell=1}^{m} \frac{r^{2}}{\varepsilon^{2}} \int_{\Omega \times \gamma_{\ell} \times D} a_{i j k l} \mathcal{T}_{\varepsilon}^{b, \ell}\left(e_{s, i j}\left(u_{\varepsilon}\right)\right) \mathcal{T}_{\varepsilon}^{b, \ell}\left(e_{s, k l}(v)\right) d x d \widehat{S} .
$$

The step-by-step construction of the unfolded limit problem (8.12) is considered in Lemmas $17,18,19$. 
Lemma 17 (The limit problem involving the limit warping) For every $\ell \in\{1, \ldots, m\}$ one has

$$
\begin{aligned}
& \int_{\Omega \times \gamma_{\ell} \times D} a_{i j k l}\left(\mathcal{E}(\mathcal{U})+\mathcal{E}_{S}(\overline{\mathcal{U}}, \widehat{\mathcal{U}}, \widehat{\mathcal{R}})+\mathcal{E}_{D}(\widetilde{u})\right)_{i j}\left(\mathcal{E}_{D}(\widetilde{v})\right)_{k l} d \widehat{S} d x=0, \\
& \forall \widetilde{v} \in L^{2}\left(\Omega \times \gamma_{\ell} ; H^{1}(D)\right)^{3} .
\end{aligned}
$$

\section{Proof Set}

$$
\begin{aligned}
& \widetilde{v}_{\varepsilon, r}(x)=\varepsilon W\left(\varepsilon \xi+\varepsilon A^{\ell}\right) V\left(\frac{s_{1}}{\varepsilon}\right) \varphi\left(\frac{s_{2}}{r}, \frac{s_{3}}{r}\right) \\
& \text { for a.e. } x=\varepsilon \xi+\varepsilon A^{\ell}+s_{1} \mathbf{t}_{1}^{\ell}+s_{2} \mathbf{t}_{2}^{\ell}+s_{3} \mathbf{t}_{3}^{\ell},\left(s_{1}, s_{2}, s_{3}\right) \in\left(0, \varepsilon l_{\ell}\right) \times D_{r}, \xi \in \Xi_{\varepsilon},
\end{aligned}
$$

where $W \in \mathcal{D}(\Omega), V \in \mathcal{D}\left(\gamma_{\ell}\right)$ and $\varphi \in H^{1}(D)^{3}, \ell \in\{1, \ldots, m\}$. Since $V$ belongs to $\mathcal{D}\left(\gamma_{\ell}\right)$ and $r / \varepsilon$ goes to 0 , the support of the above test-displacement is only included in the beams whose centerline is $\varepsilon \xi+\varepsilon \gamma_{\ell}$. Moreover, this displacement vanishes in the neighborhood of the extremities of this beam, it means that this displacement vanishes in the junction domain $\mathcal{J}_{\varepsilon, r}$.

One has

$$
\begin{aligned}
e_{s}\left(\widetilde{v}_{\varepsilon, r}\right)= & \frac{\varepsilon}{r} W\left(\varepsilon \xi+\varepsilon A^{\ell}\right) \\
& \times\left(\begin{array}{ccc}
\frac{r}{\varepsilon} \frac{d V}{d S_{1}} \varphi \cdot \mathbf{t}_{1}^{\ell} & \frac{1}{2}\left(V \frac{\partial \varphi}{\partial S_{2}} \cdot \mathbf{t}_{1}^{\ell}+\frac{r}{\varepsilon} \frac{d V}{d S_{1}} \varphi \cdot \mathbf{t}_{2}^{\ell}\right) & \frac{1}{2}\left(V \frac{\partial \varphi}{\partial S_{3}} \cdot \mathbf{t}_{1}^{\ell}+\frac{r}{\varepsilon} \frac{d V}{d S_{1}} \varphi \cdot \mathbf{t}_{3}^{\ell}\right) \\
* & V \frac{\partial \varphi}{\partial S_{2}} \cdot \mathbf{t}_{2}^{\ell} & \frac{1}{2}\left(V \frac{\partial \varphi}{\partial S_{3}} \cdot \mathbf{t}_{2}^{\ell}+V \frac{\partial \varphi}{\partial S_{2}} \cdot \mathbf{t}_{3}^{\ell}\right) \\
* & * & V \frac{\partial \varphi}{\partial S_{3}} \cdot \mathbf{t}_{3}^{\ell}
\end{array}\right)
\end{aligned}
$$

We apply the unfolding operator $\mathcal{T}_{\varepsilon}^{b, \ell}$ and pass to the limit, this gives

$$
\frac{r}{\varepsilon} \mathcal{T}_{\varepsilon}^{b, \ell}\left(e_{s}\left(\widetilde{v}_{\varepsilon, r}\right)\right) \longrightarrow W V \mathcal{E}_{D}(\varphi) \text { strongly in } L^{2}\left(\Omega \times \gamma_{\ell} \times D\right)^{3 \times 3} \text {. }
$$

Hence

$$
\begin{aligned}
& \int_{\mathcal{S}_{\varepsilon, r}} \sigma\left(u_{\varepsilon}\right): e\left(\widetilde{v}_{\varepsilon, r}\right) d x=\int_{\Omega \times \gamma_{\ell} \times D} \frac{r}{\varepsilon} \mathcal{T}_{\varepsilon}^{b, \ell}\left(\sigma_{s}\left(u_{\varepsilon}\right)\right): \frac{r}{\varepsilon} \mathcal{T}_{\varepsilon}^{b, \ell}\left(e_{s}\left(\widetilde{v}_{\varepsilon, r}\right)\right) d x \\
\rightarrow & \int_{\Omega \times \gamma_{\ell} \times D} a_{i j k l}\left(\mathcal{E}(\mathcal{U})+\mathcal{E}_{S}(\overline{\mathcal{U}}, \widehat{\mathcal{U}}, \widehat{\mathcal{R}})+\mathcal{E}_{D}(\widetilde{u})\right)_{i j} W V\left(\mathcal{E}_{D}(\varphi)\right)_{k l} d x d S .
\end{aligned}
$$

Using (5.20) and then unfolding and passing to the limit yield

$$
\begin{aligned}
& \left|\int_{\mathcal{S}_{\varepsilon, r}} \mathbf{f}_{\varepsilon} \cdot \widetilde{v}_{\varepsilon, r} d x\right|=\left|\frac{r^{2}}{\varepsilon^{2}} \int_{\Omega \times \gamma_{\ell} \times D} \mathcal{T}_{\varepsilon}^{b, \ell}\left(\mathbf{f}_{\varepsilon}\right) \cdot \mathcal{T}_{\varepsilon}^{b, \ell}\left(\widetilde{v}_{\varepsilon, r}\right) d x d \widehat{S}\right| \\
& \leq C \frac{r^{2}}{\varepsilon^{2}} \cdot \frac{\varepsilon^{2}}{r+\varepsilon^{2}}\|\mathbf{f}\|_{L^{\infty}(\Omega)}\|W\|_{L^{\infty}(\Omega)}\|V\|_{L^{\infty}\left(\gamma_{\ell}\right)}\|\varphi\|_{L^{2}(D)} \longrightarrow 0 .
\end{aligned}
$$

The above convergences lead to

$$
\int_{\Omega \times \gamma_{\ell} \times D} a_{i j k l}\left(\mathcal{E}(\mathcal{U})+\mathcal{E}_{S}(\overline{\mathcal{U}}, \widehat{\mathcal{U}}, \widehat{\mathcal{R}})+\mathcal{E}_{D}(\widetilde{u})\right)_{i j} W V\left(\mathcal{E}_{D}(\varphi)\right)_{k l} d x d \widehat{S}=0
$$


Finally, since the space $\mathcal{D}(\Omega) \otimes \mathcal{D}\left(\gamma_{\ell}\right) \otimes H^{1}(D)^{3}$ is dense in $L^{2}\left(\Omega \times \gamma_{\ell} ; H^{1}(D)\right)^{3}$ we obtain (8.1).

Lemma 18 (The limit problem involving the extensional and inextensional limit displacements) One has

$$
\begin{aligned}
& \int_{\Omega \times \mathcal{S} \times D} a_{i j k l}\left(\mathcal{E}(\mathcal{U})+\mathcal{E}_{\mathcal{S}}(\overline{\mathcal{U}}, \widehat{\mathcal{U}}, \widehat{\mathcal{R}})+\mathcal{E}_{D}(\widetilde{u})\right)_{i j}\left(\mathcal{E}_{\mathcal{S}}(\overline{\mathcal{V}}, \widehat{\mathcal{V}}, \widehat{\mathcal{B}})\right)_{k l} d x d \widehat{S} \\
= & \frac{4 \pi}{5} \int_{\Omega} G \cdot\left(\sum_{A \in \mathcal{K}} \widehat{\mathcal{B}}(\cdot, A)\right) d x+\frac{\pi}{1+\kappa} \int_{\Omega \times \mathcal{S}} \mathbf{f} \cdot \widehat{\mathcal{V}}(\cdot, \mathbf{S}) d \mathbf{S} d x, \\
& \forall(\overline{\mathcal{V}}, \widehat{\mathcal{V}}, \widehat{\mathcal{B}}) \in L^{2}\left(\Omega ; \mathcal{D}_{E x} \times \mathcal{D}_{I n}\right) .
\end{aligned}
$$

Proof Let $\phi$ be in $\mathcal{D}(\Omega)$ and $(\overline{\mathcal{V}}, \widehat{\mathcal{V}}, \widehat{\mathcal{B}})$ in $\mathcal{D}_{E X} \times \mathcal{D}_{\text {In }}$ such that $\overline{\mathcal{V}}$ and $(\widehat{\mathcal{V}}, \widehat{\mathcal{B}})$ are constant in the neighborhood of every node of $\mathcal{S}$.

Step 1. The test displacement.

Set

$$
\overline{\mathcal{V}}_{\varepsilon, r} \doteq \phi_{\varepsilon, r} \overline{\mathcal{V}}\left(\frac{\dot{-}}{\varepsilon}\right), \quad \widehat{\mathcal{V}}_{\varepsilon, r} \doteq \varepsilon \phi_{\varepsilon, r} \widehat{\mathcal{V}}(\dot{\bar{\varepsilon}}), \quad \widehat{\mathcal{B}}_{\varepsilon, r} \doteq \phi_{\varepsilon, r} \widehat{\mathcal{B}}\left(\frac{\dot{-}}{\varepsilon}\right)
$$

where $\phi_{\varepsilon, r}$ is defined in Appendix F. Since the above fields are constant in the neighborhood of every node of $\mathcal{S}_{\varepsilon}$, this allows to extend them in functions belonging to $H^{1}\left(\mathcal{S}_{\varepsilon, r}\right)$. Hence, these functions are constant in the cross-sections and in the neighborhood of every node. We remind (see Appendix F)

$$
\begin{array}{r}
\mathcal{T}_{\varepsilon}^{\mathcal{S}}\left(\phi_{\varepsilon, r}\right) \longrightarrow \phi \quad \text { strongly in } \quad L^{2}(\Omega \times \mathcal{S}), \\
\varepsilon_{\mathcal{T}_{\varepsilon}^{\mathcal{S}}}\left(\frac{d \phi_{\varepsilon, r}}{d \mathbf{s}}\right), \quad \varepsilon^{2} \mathcal{T}_{\varepsilon}^{b, \ell}\left(\frac{d^{2} \phi_{\varepsilon, r}}{d \mathbf{s}^{2}}\right) \longrightarrow 0 \quad \text { strongly in } \quad L^{2}(\Omega \times \mathcal{S}) .
\end{array}
$$

We define $v_{\varepsilon, r}$ in the beam whose centerline is $\varepsilon \xi+\varepsilon \gamma_{\ell}, \ell \in\{1, \ldots, m\}$ by

$$
\begin{aligned}
& v_{\varepsilon, r}(x)= \frac{\varepsilon^{2}}{r} \overline{\mathcal{V}}_{\varepsilon, r}\left(\varepsilon \xi+\varepsilon A^{\ell}+s_{1}\right)+\frac{\varepsilon^{2}}{r^{2}} \widehat{\mathcal{V}}_{\varepsilon, r}\left(\varepsilon \xi+\varepsilon A^{\ell}+s_{1}\right) \\
&+\frac{\varepsilon^{2}}{r^{2}} \widehat{\mathcal{B}}_{\varepsilon, r}\left(\varepsilon \xi+\varepsilon A^{\ell}+s_{1}\right) \wedge\left(s_{2} \mathbf{t}_{2}^{\ell}+s_{3} \mathbf{t}^{\ell}\right)+\widetilde{v}_{\varepsilon, r}(x), \\
& \widetilde{v}_{\varepsilon, r}(x)=-\frac{\varepsilon^{3}}{r^{2}} \frac{d \phi_{\varepsilon, r}}{d s_{1}}\left(\varepsilon \xi+\varepsilon A^{\ell}+s_{1}\right) \widehat{\mathcal{V}}\left(A^{\ell}+\frac{s_{1}}{\varepsilon}\right) \cdot\left(s_{2} \mathbf{t}_{2}^{\ell}+s_{3} \mathbf{t}_{3}^{\ell}\right) \mathbf{t}_{1}^{\ell}, \\
& \text { for a.e. } x=\varepsilon \xi+\varepsilon A^{\ell}+s_{1} \mathbf{t}_{1}^{\ell}+s_{2} \mathbf{t}_{2}^{\ell}+s_{3} \mathbf{t}_{3}^{\ell},\left(s_{1}, s_{2}, s_{3}\right) \in\left(0, \varepsilon l_{\ell}\right) \times D_{r}, \\
& \qquad \ell \in\{1, \ldots, m\}, \quad \xi \in \mathbf{\Xi}_{\varepsilon} .
\end{aligned}
$$

Observe that for every $x$ in $B\left(\varepsilon \xi+\varepsilon A^{\ell}, c_{0} r\right) \cap \mathcal{S}_{\varepsilon, r}$ one has

$$
v_{\varepsilon, r}(x)=\phi\left(\varepsilon \xi+\varepsilon A^{\ell}\right)\left[\frac{\varepsilon^{2}}{r} \overline{\mathcal{V}}\left(A^{\ell}\right)+\frac{\varepsilon^{2}}{r^{2}} \widehat{\mathcal{B}}\left(A^{\ell}\right) \wedge\left(x-\varepsilon \xi-\varepsilon A^{\ell}\right)\right] .
$$

Hence, $v_{\varepsilon, r}$ is a rigid displacement in $B\left(\varepsilon \xi+\varepsilon A^{\ell}, c_{0} r\right) \cap \mathcal{S}_{\varepsilon, r}$. This test displacement belongs to $\mathbf{V}_{\varepsilon, r}$. 
Step 2. Limit of the LHS.

One has

$$
\begin{aligned}
\frac{\partial v_{\varepsilon, r}}{\partial s_{1}} & =\frac{\varepsilon^{2}}{r} \frac{\partial \overline{\mathcal{V}}_{\varepsilon, r}}{\partial s_{1}}+\frac{\varepsilon^{2}}{r^{2}} \frac{\partial \widehat{\mathcal{V}}_{\varepsilon, r}}{\partial s_{1}}+\frac{\varepsilon^{2}}{r^{2}} \frac{\partial \widehat{\mathcal{B}}_{\varepsilon, r}}{\partial s_{1}} \wedge\left(s_{2} \mathbf{t}_{2}^{\ell}+s_{3} \mathbf{t}_{3}^{\ell}\right)+\frac{\partial \widetilde{v}_{\varepsilon, r}}{\partial s_{1}}, \\
\frac{\partial v_{\varepsilon, r}}{\partial s_{1}} & =\frac{\varepsilon^{2}}{r} \frac{d \phi_{\varepsilon, r}}{d s_{1}} \overline{\mathcal{V}}\left(\frac{\dot{-}}{\varepsilon}\right)+\frac{\varepsilon}{r} \phi_{\varepsilon, r} \frac{d \overline{\mathcal{V}}}{d S_{1}}(\dot{-})+\frac{\varepsilon^{3}}{r^{2}} \frac{d \phi_{\varepsilon, r}}{d s_{1}} \widehat{\mathcal{V}}(\dot{-})+\frac{\varepsilon^{2}}{r^{2}} \phi_{\varepsilon, r} \frac{d \widehat{\mathcal{V}}}{d S_{1}}\left(\frac{\cdot}{\varepsilon}\right) \\
& +\frac{\varepsilon}{r^{2}}\left(\varepsilon \frac{d \phi_{\varepsilon, r}}{d s_{1}} \widehat{\mathcal{B}}\left(\frac{\dot{-}}{\varepsilon}\right)+\phi_{\varepsilon, r} \frac{d \widehat{\mathcal{B}}}{d S_{1}}\left(\frac{\dot{-}}{\varepsilon}\right)\right) \wedge\left(s_{2} \mathbf{t}_{2}^{\ell}+s_{3} \mathbf{t}_{3}^{\ell}\right)+\frac{\partial \widetilde{v}_{\varepsilon, r}}{\partial s_{1}}, \\
\frac{\partial v_{\varepsilon, r}}{\partial s_{2}} & =\frac{\varepsilon^{2}}{r^{2}} \phi_{\varepsilon, r} \widehat{\mathcal{B}}\left(\frac{\dot{-}}{\varepsilon}\right) \wedge \mathbf{t}_{2}^{\ell}+\frac{\partial \widetilde{v}_{\varepsilon, r}}{\partial s_{2}}, \quad \frac{\partial v_{\varepsilon}^{\ell}}{\partial s_{3}}=\frac{\varepsilon^{2}}{r^{2}} \phi_{\varepsilon, r} \widehat{\mathcal{B}}\left(\frac{\dot{\varepsilon}}{\varepsilon}\right) \wedge \mathbf{t}_{3}^{\ell}+\frac{\partial \widetilde{v}_{\varepsilon, r}}{\partial s_{3}} .
\end{aligned}
$$

Observe that $\frac{\partial v_{\varepsilon, r}}{\partial s_{2}} \cdot \mathbf{t}_{2}=\frac{\partial v_{\varepsilon, r}}{\partial s_{3}} \cdot \mathbf{t}_{3}=\frac{\partial v_{\varepsilon, r}}{\partial s_{2}} \cdot \mathbf{t}_{3}+\frac{\partial v_{\varepsilon, r}}{\partial s_{3}} \cdot \mathbf{t}_{2}=0$ and by definition of $(\widehat{\mathcal{V}}, \widehat{\mathcal{B}}) \in \mathcal{D}_{I n}$, one has $\widehat{\mathcal{V}} \cdot \mathbf{t}_{1}=0$.

The convergences (8.6) yield

$$
\begin{array}{lrl}
\frac{r}{\varepsilon} \mathcal{T}_{\varepsilon}^{b, \ell}\left(\frac{\partial v_{\varepsilon, r}}{\partial s_{1}} \cdot \mathbf{t}_{1}^{\ell}\right) \longrightarrow \frac{\partial \overline{\mathcal{V}}}{\partial S_{1}} \cdot \mathbf{t}_{1}^{\ell}-\frac{\partial^{2} \widehat{\mathcal{V}}}{\partial S_{1}^{2}} \cdot\left(S_{2} \mathbf{t}_{2}^{\ell}+S_{3} \mathbf{t}_{3}^{\ell}\right) & \text { strongly in } & L^{2}\left(\Omega \times \gamma_{\ell} \times D\right), \\
\frac{r}{\varepsilon} \mathcal{T}_{\varepsilon}^{b, \ell}\left(\frac{\partial \widetilde{v}_{\varepsilon, r}}{\partial s_{1}}\right) \longrightarrow 0 & \text { strongly in } & L^{2}\left(\Omega \times \gamma_{\ell} \times D\right)^{3} .
\end{array}
$$

The presence of $\widetilde{v}_{\varepsilon, r}$ in the test displacement is just to eliminate $\frac{\varepsilon^{3}}{r^{2}} \frac{d \phi_{\varepsilon, r}}{d s_{1}} \widehat{\mathcal{V}}\left(\frac{\dot{\bar{\varepsilon}}}{)}\right) \cdot \mathbf{t}_{\alpha}^{\ell}$ in $\frac{\partial v_{\varepsilon, r}}{\partial s_{i}}$. $\mathbf{t}_{1}^{\ell}+\frac{\partial v_{\varepsilon, r}}{\partial s_{1}} \cdot \mathbf{t}_{i}^{\ell}, i \in\{2,3\}$. Then, again using the convergences (8.6), we obtain

$$
\underset{\varepsilon}{-} \mathcal{T}_{\varepsilon}^{b, \ell}\left(e_{s}\left(v_{\varepsilon, r}\right)\right) \longrightarrow \phi\left(\begin{array}{cccc}
\frac{\partial \overline{\mathcal{V}}}{\partial S_{1}} \cdot \mathbf{t}_{1}^{\ell}-\frac{\partial^{2} \widehat{\mathcal{V}}}{\partial S_{1}^{2}} \cdot\left(S_{2} \mathbf{t}_{2}^{\ell}+S_{3} \mathbf{t}_{3}^{\ell}\right) & * & * \\
\frac{1}{2}\left(\frac{\partial \overline{\mathcal{V}}}{\partial S_{1}} \cdot \mathbf{t}_{2}^{\ell}-S_{3} \frac{\partial \widehat{\mathcal{B}}}{\partial S_{1}} \cdot \mathbf{t}_{1}^{\ell}\right) & 0 & * \\
\frac{1}{2}\left(\frac{\partial \overline{\mathcal{V}}}{\partial S_{1}} \cdot \mathbf{t}_{3}^{\ell}+S_{2} \frac{\partial \mathcal{B}}{\partial S_{1}} \cdot \mathbf{t}_{1}^{\ell}\right) & 0 & 0
\end{array}\right)
$$

strongly in $L^{2}\left(\Omega \times \gamma_{\ell} \times D\right)^{3 \times 3}$.

Hence,

$$
{ }_{\varepsilon}^{r} \mathcal{T}_{\varepsilon}^{b, \ell}\left(e_{s}\left(v_{\varepsilon, r}\right)\right) \longrightarrow \phi\left(\mathcal{E}_{\mathcal{S}}(\overline{\mathcal{V}}, \widehat{\mathcal{V}}, \widehat{\mathcal{B}})+\mathcal{E}_{D}(\widetilde{\bar{v}})\right) \text { strongly in } \quad L^{2}\left(\Omega \times \gamma_{\ell} \times D\right)^{3 \times 3}
$$

where

$$
\widetilde{\bar{v}}=S_{2}\left(\frac{\partial \overline{\mathcal{V}}}{\partial S_{1}} \cdot \mathbf{t}_{2}\right) \mathbf{t}_{1}+S_{3}\left(\frac{\partial \overline{\mathcal{V}}}{\partial S_{1}} \cdot \mathbf{t}_{3}\right) \mathbf{t}_{1} .
$$

Unfolding the left-hand side of (5.8) and passing to the limit give

$$
\begin{aligned}
& \int_{\mathcal{S}_{\varepsilon, r}} \sigma\left(u_{\varepsilon}\right): e\left(v_{\varepsilon, r}\right) d x=\sum_{\ell=1}^{m} \int_{\Omega \times \gamma_{\ell} \times D} \frac{r}{\varepsilon} \mathcal{T}_{\varepsilon}^{b, \ell}\left(\sigma_{s}\left(u_{\varepsilon}\right)\right): \frac{r}{\varepsilon} \mathcal{T}_{\varepsilon}^{b, \ell}\left(e_{s}\left(v_{\varepsilon, r}\right)\right) d x \\
& \rightarrow \int_{\Omega \times \mathcal{S} \times D} a_{i j k l}\left(\mathcal{E}(\mathcal{U})+\mathcal{E}_{\mathcal{S}}(\overline{\mathcal{U}}, \widehat{\mathcal{U}}, \widehat{\mathcal{R}})+\mathcal{E}_{D}(\widetilde{u})\right)_{i j} \phi\left(\mathcal{E}_{\mathcal{S}}(\overline{\mathcal{V}}, \widehat{\mathcal{V}}, \widehat{\mathcal{B}})+\mathcal{E}_{D}(\widetilde{\bar{v}})\right)_{k l} d x d \widehat{S}
\end{aligned}
$$


Step 3. Limit of the RHS.

Now, we consider the right-hand side of (5.8)

$$
\int_{\mathcal{S}_{\varepsilon, r}} f_{\varepsilon} \cdot v_{\varepsilon, r} d x=\sum_{A \in \mathcal{K}_{\varepsilon}} \int_{B(A, r)} F_{r, \mathcal{K}_{\varepsilon}} \cdot v_{\varepsilon, r} d x+\int_{\mathcal{S}_{\varepsilon, r}} \mathbf{f}_{\varepsilon} \cdot v_{\varepsilon, r} d x
$$

Let's take the first term in the right-hand side of (8.8). Taking into account the symmetries of the ball $B\left(\varepsilon \xi+\varepsilon A^{\ell}, r\right)$ and the fact that $\int_{B(O, r)}|x|^{2} d x=\frac{4 \pi r^{5}}{5}$. After a straightforward calculation, one obtains

$$
\begin{aligned}
& \sum_{A \in \mathcal{K}_{\varepsilon}} \int_{B(A, r)} F_{r, \mathcal{K}_{\varepsilon}} \cdot v_{\varepsilon, r} d x \\
& =\sum_{A^{\ell} \in \mathcal{K}} \sum_{\xi \in \Xi_{\varepsilon}} \int_{B\left(\varepsilon \xi+\varepsilon A^{\ell}, r\right)}\left[\frac{\varepsilon^{2}}{r^{2}} F\left(\varepsilon \xi+\varepsilon A^{\ell}\right)+\frac{\varepsilon}{r^{3}} G\left(\varepsilon \xi+\varepsilon A^{\ell}\right) \wedge\left(x-\left(\varepsilon \xi-\varepsilon A^{\ell}\right)\right]\right. \\
& =\frac{4 \pi}{3} \varepsilon^{4} \sum_{A^{\ell} \in \mathcal{K}} \sum_{\xi \in \Xi_{\varepsilon}} \phi\left(\varepsilon \xi+\varepsilon A^{\ell}\right) F\left(\varepsilon \xi+\varepsilon A^{\ell}\right) \cdot \overline{\mathcal{V}}\left(A^{\ell}\right) \\
& \quad+\frac{4 \pi}{5} \varepsilon^{3} \sum_{A^{\ell} \in \mathcal{K}} \sum_{\xi \in \Xi_{\varepsilon}} \phi\left(\varepsilon \xi+\varepsilon A^{\ell}\right) G\left(\varepsilon \xi+\varepsilon A^{\ell}\right) \cdot \widehat{\mathcal{B}}\left(A^{\ell}\right) .
\end{aligned}
$$

Since $|Y|=1$, one has

$$
\begin{aligned}
& \sum_{A^{\ell} \in \mathcal{K}} \sum_{\xi \in \Xi_{\varepsilon}} \varepsilon^{3} \phi\left(\varepsilon \xi+\varepsilon A^{\ell}\right) F\left(\varepsilon \xi+\varepsilon A^{\ell}\right) \cdot \overline{\mathcal{V}}\left(A^{\ell}\right) \longrightarrow \int_{\Omega} F \cdot \phi\left(\sum_{A \in \mathcal{K}} \overline{\mathcal{V}}(A)\right) d x \\
& \sum_{A^{\ell} \in \mathcal{K}} \sum_{\xi \in \Xi_{\varepsilon}} \varepsilon^{3} \phi\left(\varepsilon \xi+\varepsilon A^{\ell}\right) G\left(\varepsilon \xi+\varepsilon A^{\ell}\right) \cdot \widehat{\mathcal{B}}\left(A^{\ell}\right) \longrightarrow \int_{\Omega} G \cdot \phi\left(\sum_{A \in \mathcal{K}} \widehat{\mathcal{B}}(A)\right) d x .
\end{aligned}
$$

Hence,

$$
\sum_{A \in \mathcal{K}_{\varepsilon}} \int_{B(A, r)} F_{r, \mathcal{K}_{\varepsilon}} \cdot v_{\varepsilon, r} d x \longrightarrow \frac{4 \pi}{5} \int_{\Omega} G \cdot \phi\left(\sum_{A \in \mathcal{K}} \widehat{\mathcal{B}}(A)\right) d x
$$

Now, we take the second term in the right-hand side of (8.8).

Due to (6.6), we only need to consider $\frac{r^{2}}{\varepsilon^{2}} \sum_{\ell=1}^{m} \int_{\Omega \times \gamma_{\ell} \times D} \mathcal{T}_{\varepsilon}^{b, \ell}\left(\mathbf{f}_{\varepsilon}\right) \cdot \mathcal{T}_{\varepsilon}^{b, \ell}\left(v_{\varepsilon, r}\right) d x d \widehat{S}$. One has

$$
\begin{aligned}
\frac{r^{2}}{\varepsilon^{2}} \sum_{\ell=1}^{m} \int_{\Omega \times \gamma_{\ell} \times D} \mathcal{T}_{\varepsilon}^{b, \ell}\left(\mathbf{f}_{\varepsilon}\right) \cdot \mathcal{T}_{\varepsilon}^{b, \ell}\left(v_{\varepsilon, r}\right) \quad d x d \widehat{S} \\
=\frac{r^{2}}{\varepsilon^{2}} \frac{\varepsilon}{r+\varepsilon^{2}} \frac{\varepsilon^{2}}{r^{2}} \sum_{\ell=1}^{m} \int_{\Omega \times \gamma_{\ell} \times D} \mathcal{T}_{\varepsilon}^{b, \ell}(\mathbf{f}) \\
\cdot\left[r \mathcal{T}_{\varepsilon}^{b, \ell}\left(\overline{\mathcal{V}}_{\varepsilon, r}\right)+\mathcal{T}_{\varepsilon}^{b, \ell}\left(\widehat{\mathcal{V}}_{\varepsilon, r}\right)+r \mathcal{T}_{\varepsilon}^{b, \ell}\left(\widehat{\mathcal{B}}_{\varepsilon, r}\right) \wedge\left(S_{2} \mathbf{t}_{2}^{\ell}+S_{3} \mathbf{t}_{3}^{\ell}\right)\right] d x d \widehat{S}
\end{aligned}
$$


Assumptions (7.1) and convergence (8.6) 1 lead to

$$
\begin{aligned}
& \frac{\varepsilon^{2}}{r+\varepsilon^{2}} \sum_{\ell=1}^{m} \int_{\Omega \times \gamma_{\ell} \times D} \mathcal{T}_{\varepsilon}^{b, \ell}(\mathbf{f}) \cdot \frac{1}{\varepsilon} \mathcal{T}_{\varepsilon}^{b, \ell}\left(\widehat{\mathcal{V}}_{\varepsilon, r}\right) \quad d x d \widehat{S} \longrightarrow \frac{\pi}{1+\kappa} \int_{\Omega \times \mathcal{S}} \mathbf{f}(x) \cdot \phi(x) \widehat{\mathcal{V}}(\mathbf{S}) d x d \mathbf{S} \\
& \frac{\varepsilon r}{r+\varepsilon^{2}} \sum_{\ell=1}^{m} \int_{\Omega \times \gamma_{\ell} \times D} \mathcal{T}_{\varepsilon}^{b, \ell}(\mathbf{f}) \cdot \mathcal{T}_{\varepsilon}^{b, \ell}\left(\overline{\mathcal{V}}_{\varepsilon, r}\right) \quad d \widehat{S} d x \longrightarrow 0, \\
& \frac{\varepsilon r}{r+\varepsilon^{2}} \sum_{\ell=1}^{m} \int_{\Omega \times \gamma_{\ell} \times D} \mathcal{T}_{\varepsilon}^{b, \ell}\left(\mathbf{f}_{\varepsilon}\right) \cdot\left[\mathcal{T}_{\varepsilon}^{b, \ell}\left(\widehat{\mathcal{B}}_{\varepsilon, r}\right) \wedge\left(S_{2} \mathbf{t}_{2}^{\ell}+S_{3} \mathbf{t}_{3}^{\ell}\right)\right] d x d \widehat{S} \longrightarrow 0 .
\end{aligned}
$$

Hence,

$$
\int_{\mathcal{S}_{\varepsilon, r}} f_{\varepsilon} \cdot v_{\varepsilon, r} d x \longrightarrow \frac{4 \pi}{5} \int_{\Omega} G \cdot \phi\left(\sum_{A \in \mathcal{K}} \widehat{\mathcal{B}}(A)\right) d x+\frac{\pi}{1+\kappa} \int_{\Omega \times \mathcal{S}} \mathbf{f} \cdot \phi \widehat{\mathcal{V}}(\mathbf{S}) d x d \mathbf{S}
$$

Lemma 24 and the density of $\mathcal{D}(\Omega) \otimes \mathcal{D}_{E x}$ in $L^{2}\left(\Omega ; \mathcal{D}_{E x}\right)$ and $\mathcal{D}(\Omega) \otimes \mathcal{D}_{I n}$ in $L^{2}\left(\Omega ; \mathcal{D}_{\text {In }}\right)$ lead to

$$
\begin{aligned}
& \int_{\Omega \times \mathcal{S} \times D} a_{i j k l}\left(\mathcal{E}(\mathcal{U})+\mathcal{E}_{\mathcal{S}}(\overline{\mathcal{U}}, \widehat{\mathcal{U}}, \widehat{\mathcal{R}})+\mathcal{E}_{D}(\widetilde{u})\right)_{i j}\left(\mathcal{E}_{\mathcal{S}}(\overline{\mathcal{V}}, \widehat{\mathcal{V}}, \widehat{\mathcal{B}})+\mathcal{E}_{D}(\widetilde{\bar{v}})\right)_{k l} d x d \widehat{S} \\
= & \frac{4 \pi}{5} \int_{\Omega} G \cdot\left(\sum_{A \in \mathcal{K}} \widehat{\mathcal{B}}(\cdot, A)\right) d x+\frac{\pi}{1+\kappa} \int_{\Omega \times \mathcal{S}} \mathbf{f} \cdot \widehat{\mathcal{V}}(\cdot, \mathbf{S}) d x d \mathbf{S} \\
& \forall(\overline{\mathcal{V}}, \widehat{\mathcal{V}}, \widehat{\mathcal{B}}) \in L^{2}\left(\Omega ; \mathcal{D}_{E x} \times \mathcal{D}_{I n}\right) .
\end{aligned}
$$

Besides, since $\widetilde{\bar{v}}$ belongs to $L^{2}\left(\Omega \times \mathcal{S} ; H^{1}(D)\right)^{3}$ equality (8.1) together with the one above yield (8.5).

Lemma 19 (The limit problem involving the macroscopic limit displacement) One has

$$
\begin{aligned}
& \int_{\Omega \times \mathcal{S} \times D} a_{i j k l}\left(\mathcal{E}(\mathcal{U})+\mathcal{E}_{\mathcal{S}}(\overline{\mathcal{U}}, \widehat{\mathcal{U}}, \widehat{\mathcal{R}})+\mathcal{E}_{D}(\widetilde{u})\right)_{i j}(\mathcal{E}(\mathcal{V}))_{k l} d x d \widehat{S} \\
& =\frac{4 \pi|\mathcal{K}|}{3} \int_{\Omega} F \cdot \mathcal{V} d x+\frac{\kappa|\mathcal{S}|}{1+\kappa} \int_{\Omega} \mathbf{f} \cdot \mathcal{V} d x, \quad \forall \mathcal{V} \in H_{\Gamma}^{1}(\Omega)^{3},
\end{aligned}
$$

where $^{2}|\mathcal{K}|$ is the number of points of $\mathcal{K}$ and $\mathcal{S}$ the measure of $\mathcal{S}$.

Proof Step 1. Limit of the LHS of (5.8).

Let $\mathcal{V}$ be in $\mathcal{D}\left(\mathbb{R}^{3}\right)^{3}$ such that $\mathcal{V}=0$ in $\Omega^{\prime} \backslash \bar{\Omega}$. We define $\mathcal{V}_{\varepsilon, r}$ using $\mathrm{F}$. This function is extended as in Step 1 of the proof of Lemma 18. Set

$$
v_{\varepsilon, r}=\frac{\varepsilon}{r} \mathcal{V}_{\varepsilon, r} \in \mathbf{V}_{\varepsilon, r}
$$

${ }^{2}$ Here, by convention $\frac{+\infty}{1+\infty}=1$. 
We have

$$
\begin{aligned}
& { }_{\varepsilon}^{r} \mathcal{T}_{\varepsilon}^{b, \ell}\left(v_{\varepsilon, r}\right) \longrightarrow \mathcal{V} \quad \text { strongly in } \quad L^{2}\left(\Omega \times \gamma_{\ell} \times D\right)^{3}, \\
& \text { and } \quad{ }^{r} \mathcal{T}_{\varepsilon}^{b, \ell}\left(e_{s}\left(v_{\varepsilon, r}\right)\right) \longrightarrow\left(\begin{array}{ccc}
\left(\nabla \mathcal{V} \mathbf{t}_{1}^{\ell}\right) \cdot \mathbf{t}_{1}^{\ell} & * & * \\
\frac{1}{2}\left(\nabla \mathcal{V} \mathbf{t}_{1}^{\ell}\right) \cdot \mathbf{t}_{2}^{\ell} & 0 & 0 \\
\frac{1}{2}\left(\nabla \mathcal{V} \mathbf{t}_{1}^{\ell}\right) \cdot \mathbf{t}_{3}^{\ell} & 0 & 0
\end{array}\right)=\mathcal{E}(\mathcal{V})+\mathcal{E}_{D}(\widetilde{\widetilde{v}}) \\
& \text { strongly in } L^{2}\left(\Omega \times \gamma_{\ell} \times D\right)^{3 \times 3}
\end{aligned}
$$

where

$$
\widetilde{\widetilde{v}}=S_{2}\left(\left(\nabla \mathcal{V} \mathbf{t}_{1}\right) \cdot \mathbf{t}_{2}\right) \mathbf{t}_{1}+S_{3}\left(\left(\nabla \mathcal{V} \mathbf{t}_{1}\right) \cdot \mathbf{t}_{3}\right) \mathbf{t}_{1}, \quad \text { a.e. in } \Omega \times \mathcal{S} \times D
$$

Convergence (8.11) leads to

$$
\begin{aligned}
& \int_{\mathcal{S}_{\varepsilon, r}} \sigma\left(u_{\varepsilon}\right): e(\mathcal{V}) d x \\
& \quad \longrightarrow \int_{\Omega \times \mathcal{S} \times D} a_{i j k l}\left(\mathcal{E}(\mathcal{U})+\mathcal{E}_{\mathcal{S}}(\overline{\mathcal{U}}, \widehat{\mathcal{U}}, \widehat{\mathcal{R}})+\mathcal{E}_{D}(\widetilde{u})\right)_{i j}\left(\mathcal{E}(\mathcal{V})+\mathcal{E}_{D}(\widetilde{\widetilde{v}})\right)_{k l} d x d \widehat{S}
\end{aligned}
$$

Step 2. Limit of the RHS.

Now we consider the right-hand side of (5.8). By (5.20), firstly we have

$$
\begin{aligned}
& \sum_{A \in \mathcal{K}_{\varepsilon}} \int_{B(A, r)} F_{r, \mathcal{K}_{\varepsilon}} \cdot v_{\varepsilon, r} d x \\
& \quad=\sum_{\mathcal{A} \in \mathcal{K}_{\varepsilon}} \int_{B(\mathcal{A}, r)}\left(\frac{\varepsilon^{2}}{r^{2}} F(\mathcal{A})+\frac{\varepsilon}{r^{3}} G(\mathcal{A}) \wedge(x-\mathcal{A})\right) \cdot \frac{\varepsilon}{r} \mathcal{V}(\mathcal{A}) d x \\
& \quad=\frac{4 \pi}{3} \sum_{A \in \mathcal{K}} \sum_{\xi \in \Xi_{\varepsilon}} F(\varepsilon \xi+\varepsilon A) \cdot \mathcal{V}(\varepsilon \xi+\varepsilon A) \varepsilon^{3} \longrightarrow \frac{4 \pi|\mathcal{K}|}{3} \int_{\Omega} F \cdot \mathcal{V} d x
\end{aligned}
$$

and secondly, due to (6.6), we pass to the limit in

$$
\frac{r^{2}}{\varepsilon^{2}} \frac{\varepsilon}{r+\varepsilon^{2}} \frac{\varepsilon}{r} \sum_{\ell=1}^{m} \int_{\Omega \times \gamma_{\ell} \times D} \mathcal{T}_{\varepsilon}^{b, \ell}\left(\mathbf{f}_{\varepsilon}\right) \cdot \mathcal{T}_{\varepsilon}^{b, \ell}\left(\mathcal{V}_{\varepsilon, r}\right) d x d S \longrightarrow \frac{\kappa|\mathcal{S}|}{1+\kappa} \int_{\Omega} \mathbf{f} \cdot \mathcal{V} d x
$$

Hence

$$
\begin{aligned}
\int_{\mathcal{S}_{\varepsilon, r}} f_{\varepsilon} \cdot v_{\varepsilon, r} & =\sum_{A \in \mathcal{K}_{\varepsilon}} \int_{B(A, r)} F_{r, \mathcal{K}_{\varepsilon}} \cdot v_{\varepsilon, r} d x+\int_{\mathcal{S}_{\varepsilon, r}} \mathbf{f}_{\varepsilon} \cdot v_{\varepsilon, r} d x \\
& \longrightarrow \frac{4 \pi|\mathcal{K}|}{3} \int_{\Omega} F \cdot \mathcal{V} d x+\frac{\kappa|\mathcal{S}|}{1+\kappa} \int_{\Omega} \mathbf{f} \cdot \mathcal{V} d x
\end{aligned}
$$

Since the set of functions belonging to $\mathcal{D}\left(\mathbb{R}^{3}\right)^{3}$ and vanishing in $\Omega^{\prime} \backslash \Omega$ is dense in $H_{\Gamma}^{1}(\Omega)^{3}$, we obtain

$$
\begin{aligned}
& \int_{\Omega \times \mathcal{S} \times D} a_{i j k l}\left(\mathcal{E}(\mathcal{U})+\mathcal{E}_{\mathcal{S}}(\overline{\mathcal{U}}, \widehat{\mathcal{U}}, \widehat{\mathcal{R}})+\mathcal{E}_{D}(\widetilde{u})\right)_{i j}\left(\mathcal{E}(\mathcal{V})+\mathcal{E}_{D}(\widetilde{\widetilde{v}})\right)_{k l} d x d \widehat{S} \\
= & \frac{4 \pi|\mathcal{K}|}{3} \int_{\Omega} F \cdot \mathcal{V} d x+\frac{\kappa|\mathcal{S}|}{1+\kappa} \int_{\Omega} \mathbf{f} \cdot \mathcal{V} d x, \quad \forall \mathcal{V} \in H_{\Gamma}^{1}(\Omega)^{3} .
\end{aligned}
$$


Taking into account that $\widetilde{\widetilde{v}}$ belongs to $L^{2}\left(\Omega \times \mathcal{S} ; H^{1}(D)\right)^{3}$ and using (8.1), equality (8.10) is proved.

Theorem 3 (The unfolded limit problem) Let $u_{\varepsilon}$ be the solution to (5.8). There exist $\mathcal{U} \in$ $H_{\Gamma}^{1}(\Omega)^{3},(\overline{\mathcal{U}}, \widehat{\mathcal{U}}, \widehat{\mathcal{R}}) \in L^{2}\left(\Omega ; \mathcal{D}_{E x} \times \mathcal{D}_{\text {In }}\right)$ and $\tilde{u} \in L^{2}\left(\Omega \times \mathcal{S} ; \mathcal{D}_{w}\right)$ such that $(\mathcal{U}, \overline{\mathcal{U}}, \widehat{\mathcal{U}}, \widehat{\mathcal{R}}, \widetilde{u})$ is the solution to the following unfolded problem:

$$
\begin{aligned}
& \int_{\Omega \times \mathcal{S} \times D} a_{i j k l}\left(\mathcal{E}(\mathcal{U})+\mathcal{E}_{\mathcal{S}}(\overline{\mathcal{U}}, \widehat{\mathcal{U}}, \widehat{\mathcal{R}})+\mathcal{E}_{D}(\widetilde{u})\right)_{i j}\left(\mathcal{E}(\mathcal{V})+\mathcal{E}_{\mathcal{S}}(\overline{\mathcal{V}}, \widehat{\mathcal{V}}, \widehat{\mathcal{B}})+\mathcal{E}_{D}(\widetilde{v})\right)_{k l} d x d \widehat{S} \\
= & \frac{4 \pi|\mathcal{K}|}{3} \int_{\Omega} F \cdot \mathcal{V} d x+\frac{4 \pi}{5} \int_{\Omega} G \cdot\left(\sum_{A \in \mathcal{K}} \widehat{\mathcal{B}}(\cdot, A)\right) d x+\frac{\kappa|\mathcal{S}|}{1+\kappa} \int_{\Omega} \mathbf{f} \cdot \mathcal{V} d x \\
& +\frac{\pi}{1+\kappa} \int_{\Omega \times \mathcal{S}} \mathbf{f} \cdot \widehat{\mathcal{V}}(\cdot, \mathbf{S}) d x d \mathbf{S} \\
& \forall \mathcal{V} \in H_{\Gamma}^{1}(\Omega)^{3}, \quad \forall(\overline{\mathcal{V}}, \widehat{\mathcal{V}}, \widehat{\mathcal{B}}) \in L^{2}\left(\Omega ; \mathcal{D}_{E x} \times \mathcal{D}_{I n}\right), \quad \forall \widetilde{v} \in L^{2}\left(\Omega \times \mathcal{S} ; \mathcal{D}_{w}\right) .
\end{aligned}
$$

Moreover, the following convergences hold $(\ell \in\{1, \ldots, m\})$ :

$$
\frac{r}{\varepsilon} \mathcal{T}_{\varepsilon}^{b, \ell}\left(e_{s}\left(u_{\varepsilon}\right)\right) \longrightarrow \mathcal{E}(\mathcal{U})+\mathcal{E}_{S}(\overline{\mathcal{U}}, \widehat{\mathcal{U}}, \widehat{\mathcal{R}})+\mathcal{E}_{D}(\widetilde{u}) \quad \text { strongly in } \quad L^{2}\left(\Omega \times \gamma_{\ell} \times D\right)^{3 \times 3} .
$$

Denote

$$
\begin{aligned}
& \mathbf{M}^{11}=\left(\begin{array}{lll}
1 & 0 & 0 \\
0 & 0 & 0 \\
0 & 0 & 0
\end{array}\right), \quad \mathbf{M}^{22}=\left(\begin{array}{lll}
0 & 0 & 0 \\
0 & 1 & 0 \\
0 & 0 & 0
\end{array}\right), \quad \mathbf{M}^{33}=\left(\begin{array}{lll}
0 & 0 & 0 \\
0 & 0 & 0 \\
0 & 0 & 1
\end{array}\right), \\
& \mathbf{M}^{12}=\mathbf{M}^{21}=\frac{1}{2}\left(\begin{array}{lll}
0 & 1 & 0 \\
1 & 0 & 0 \\
0 & 0 & 0
\end{array}\right), \quad \mathbf{M}^{13}=\mathbf{M}^{31}=\frac{1}{2}\left(\begin{array}{lll}
0 & 0 & 1 \\
0 & 0 & 0 \\
1 & 0 & 0
\end{array}\right), \\
& \mathbf{M}^{23}=\mathbf{M}^{32}=\frac{1}{2}\left(\begin{array}{lll}
0 & 0 & 0 \\
0 & 0 & 1 \\
0 & 1 & 0
\end{array}\right) .
\end{aligned}
$$

Proof From Lemmas $17,18,19$ we obtain that $(\mathcal{U}, \overline{\mathcal{U}}, \widehat{\mathcal{U}}, \widehat{\mathcal{R}}, \widetilde{u})$ satisfies $(8.12)$ for every test function $\mathcal{V} \in H_{\Gamma}^{1}(\Omega)^{3},(\overline{\mathcal{V}}, \widehat{\mathcal{V}}, \widehat{\mathcal{B}}) \in L^{2}\left(\Omega ; \mathcal{D}_{E x} \times \mathcal{D}_{I n}\right)$ and $\widetilde{w} \in L^{2}\left(\Omega \times \mathcal{S} ; \mathcal{D}_{w}\right) \subset L^{2}(\Omega \times$ $\left.\mathcal{S} ; H^{1}(D)\right)^{3}$.

The coercivity of this problem is given by Lemma 26. Since the problem (8.12) admits a unique solution, the whole sequences in Theorems 1, 2 and (8.13) converge to their limits.

Now, we prove the strong convergence (8.13). First, observe that due to the inclusion of $\mathcal{J}_{\varepsilon, r}$ in $\bigcup_{A \in \mathcal{K}_{\varepsilon}} B\left(A, c_{0} r\right)$ given by (5.1), the portions of beams which correspond to $S_{1} \in$ $\left(2 c_{0} r, l_{\ell}-2 c_{0} r\right)$ are all disjoint. Furthermore, since $\sigma\left(u_{\varepsilon}\right): e\left(u_{\varepsilon}\right)$ is non-negative, one has

$$
\begin{aligned}
& \frac{r^{2}}{\varepsilon^{2}} \sum_{\ell=1}^{m} \int_{\Omega \times\left(0, l_{\ell}\right) \times D} \mathcal{T}_{\varepsilon}^{b, \ell}\left(\sigma_{s}\left(u_{\varepsilon}\right)\right): \mathcal{T}_{\varepsilon}^{b, \ell}\left(e_{s}\left(u_{\varepsilon}\right)\right) \mathbf{1}_{\left(2 c_{0} r, l_{\ell}-2 c_{0} r\right)} d x d \widehat{S} \\
& \quad \leq \liminf _{\varepsilon \rightarrow 0} \int_{\mathcal{S}_{\varepsilon, r}} \sigma\left(u_{\varepsilon}\right): e\left(u_{\varepsilon}\right) d x .
\end{aligned}
$$


From (7.13) and the fact that $r$ goes to 0 , one obtains $(\ell \in\{1, \ldots, m\})$

$$
\begin{aligned}
& { }_{\varepsilon}^{r} \mathcal{T}_{\varepsilon}^{b, \ell}\left(e_{S}\left(u_{\varepsilon}\right)\right) \mathbf{1}_{\left(2 c_{0} r, l_{\ell}-2 c_{0} r\right)} \rightarrow \mathcal{E}(\mathcal{U})+\mathcal{E}_{S}(\overline{\mathcal{U}}, \widehat{\mathcal{U}}, \widehat{\mathcal{R}})+\mathcal{E}_{D}(\widetilde{u}) \\
& \quad \text { weakly in } L^{2}\left(\Omega \times \gamma_{\ell} \times D\right)^{3 \times 3} .
\end{aligned}
$$

Hence, choosing $u_{\varepsilon}$ as a test function in (5.8) and using a weak lower semi-continuity of convex functionals, one has

$$
\begin{aligned}
& \int_{\Omega \times \mathcal{S} \times D} a_{i j k l}\left(\mathcal{E}(\mathcal{U})+\mathcal{E}_{\mathcal{S}}(\overline{\mathcal{U}}, \widehat{\mathcal{U}}, \widehat{\mathcal{R}})+\mathcal{E}_{D}(\widetilde{u})\right)_{i j}\left(\mathcal{E}(\mathcal{U})+\mathcal{E}_{\mathcal{S}}(\overline{\mathcal{U}}, \widehat{\mathcal{U}}, \widehat{\mathcal{B}})+\mathcal{E}_{D}(\widetilde{u})\right)_{k l} d x d \widehat{S} \\
\leq & \liminf _{\varepsilon \rightarrow 0} \frac{r^{2}}{\varepsilon^{2}} \sum_{\ell=1}^{m} \int_{\Omega \times\left(0, l_{\ell}\right) \times D} \mathcal{T}_{\varepsilon}^{b, \ell}\left(a_{i j k l}^{\varepsilon}\right) \mathcal{T}_{\varepsilon}^{b, \ell}\left(e_{s, i j}\left(u_{\varepsilon}\right)\right) \mathcal{T}_{\varepsilon}^{b, \ell}\left(e_{s, k l}\left(u_{\varepsilon}\right)\right) \mathbf{1}_{\left(2 c_{0} r, l_{\ell}-2 c_{0} r\right)} d x d \widehat{S} \\
\leq & \liminf _{\varepsilon \rightarrow 0} \int_{\mathcal{S}_{\varepsilon, r}} \sigma\left(u_{\varepsilon}\right): e\left(u_{\varepsilon}\right) d x \leq \limsup _{\varepsilon \rightarrow 0} \int_{\mathcal{S}_{\varepsilon, r}} \sigma\left(u_{\varepsilon}\right): e\left(u_{\varepsilon}\right) d x=\limsup _{\varepsilon \rightarrow 0} \int_{\mathcal{S}_{\varepsilon, r}} f_{\varepsilon} \cdot u_{\varepsilon} d x \\
= & \frac{4 \pi|\mathcal{K}|}{3} \int_{\Omega} F \cdot \mathcal{U} d x+\frac{4 \pi}{5} \int_{\Omega} G \cdot\left(\sum_{A \in \mathcal{K}} \widehat{\mathcal{R}}(\cdot, A)\right) d x \\
& +\frac{\kappa|\mathcal{S}|}{1+\kappa} \int_{\Omega} \mathbf{f} \cdot \mathcal{U} d x+\frac{\pi}{1+\kappa} \int_{\Omega \times \mathcal{S}} \mathbf{f} \cdot \widehat{\mathcal{U}}(\cdot, \mathbf{S}) d x d \mathbf{S}, \\
= & \int_{\Omega \times \mathcal{S} \times D} a_{i j k l}\left(\mathcal{E}(\mathcal{U})+\mathcal{E}_{\mathcal{S}}(\overline{\mathcal{U}}, \widehat{\mathcal{U}}, \widehat{\mathcal{R}})+\mathcal{E}_{D}(\widetilde{u})\right)_{i j}\left(\mathcal{E}(\mathcal{U})+\mathcal{E}_{\mathcal{S}}(\overline{\mathcal{U}}, \widehat{\mathcal{U}}, \widehat{\mathcal{B}})+\mathcal{E}_{D}(\widetilde{u})\right)_{k l} d x d \widehat{S} .
\end{aligned}
$$

Thus, all inequalities above are equalities and

$$
\begin{aligned}
& \lim _{\varepsilon \rightarrow 0} \int_{\mathcal{S}_{\varepsilon, r}} \sigma\left(u_{\varepsilon}\right): e\left(u_{\varepsilon}\right) d x \\
& \quad=\int_{\Omega \times \mathcal{S} \times D} a_{i j k l}\left(\mathcal{E}(\mathcal{U})+\mathcal{E}_{\mathcal{S}}(\overline{\mathcal{U}}, \widehat{\mathcal{U}}, \widehat{\mathcal{R}})+\mathcal{E}_{D}(\widetilde{u})\right)_{i j}\left(\mathcal{E}(\mathcal{U})+\mathcal{E}_{\mathcal{S}}(\overline{\mathcal{U}}, \widehat{\mathcal{U}}, \widehat{\mathcal{B}})+\mathcal{E}_{D}(\widetilde{u})\right)_{k l} d x d \widehat{S},
\end{aligned}
$$

which in turn leads to the strong convergence (8.13).

\section{The Homogenized Problem}

\subsection{Expression of the Warping $\tilde{\boldsymbol{u}}$}

In this subsection we give the expression of the warping $\tilde{u}$ in terms of the macroscopic displacement $\mathcal{U}$ and the microscopic fields $\overline{\mathcal{U}}, \widehat{\mathcal{U}}, \widehat{\mathcal{R}}$.

To this end, we use the variational formulation (8.1). For every $\ell \in\{1, \ldots, m\}$ one has

$$
\int_{D} a_{i j k l}\left(\mathcal{E}_{D}(\widetilde{u})\right)_{i j}\left(\mathcal{E}_{D}(\widetilde{v})\right)_{k l} d S_{2} d S_{3}=-\int_{D} a_{i j k l}\left(\mathcal{E}(\mathcal{U})+\mathcal{E}_{S}(\overline{\mathcal{U}}, \widehat{\mathcal{U}}, \widehat{\mathcal{R}})\right)_{i j}\left(\mathcal{E}_{D}(\widetilde{v})\right)_{k l} d S_{2} d S_{3},
$$

a.e. in $\Omega \times \gamma_{\ell}, \quad \forall \widetilde{v} \in \mathcal{D}_{w}$.

This shows that $\tilde{u}$ can be expressed in terms of the elements of the tensors $\mathcal{E}$ and $\mathcal{E}_{\mathcal{S}}$. 
We write

$$
\begin{aligned}
\mathcal{E}(\mathcal{U})+ & \mathcal{E}_{S}(\overline{\mathcal{U}}, \widehat{\mathcal{V}}, \widehat{\mathcal{R}}) \\
= & \left(\begin{array}{ccc}
\left(e(\mathcal{U}) \mathbf{t}_{1}\right) \cdot \mathbf{t}_{1} & 0 & 0 \\
0 & 0 & 0 \\
0 & 0 & 0
\end{array}\right) \\
& +\left(\begin{array}{ccc}
\frac{\partial \overline{\mathcal{U}}}{\partial S_{1}} \cdot \mathbf{t}_{1}-\frac{\partial^{2} \widehat{\mathcal{U}}}{\partial S_{1}^{2}} \cdot\left(S_{2} \mathbf{t}_{2}+S_{3} \mathbf{t}_{3}\right) & * & * \\
-\frac{S_{3}}{2} \frac{\partial \widehat{\mathcal{R}}}{\partial S_{1}} \cdot \mathbf{t}_{1} & 0 & 0 \\
\frac{S_{2}}{2} \frac{\partial \widehat{\mathcal{R}}}{\partial S_{1}} \cdot \mathbf{t}_{1} & 0
\end{array}\right) \\
= & \left(\left(e(\mathcal{U}) \mathbf{t}_{1}\right) \cdot \mathbf{t}_{1}+\frac{\partial \overline{\mathcal{U}}}{\partial S_{1}} \cdot \mathbf{t}_{1}\right) \mathbf{M}^{11}-\sum_{\alpha=2}^{3} \frac{\partial^{2} \widehat{\mathcal{U}}}{\partial S_{1}^{2}} \cdot \mathbf{t}_{\alpha} S_{\alpha} \mathbf{M}^{11}+\frac{\partial \overline{\mathcal{R}}}{\partial S_{1}} \cdot \mathbf{t}_{1}\left(S_{2} \mathbf{M}^{13}-S_{3} \mathbf{M}^{12}\right) \\
= & \left(\left(e(\mathcal{U}) \mathbf{t}_{1}\right) \cdot \mathbf{t}_{1}+\frac{\partial \overline{\mathcal{U}}}{\partial S_{1}} \cdot \mathbf{t}_{1}\right) \mathbf{M}^{11}+\frac{\partial \widehat{\mathcal{R}}}{\partial S_{1}} \cdot \mathbf{t}_{1}\left(S_{2} \mathbf{M}^{13}-S_{3} \mathbf{M}^{12}\right) \\
& +\left(\frac{\partial \widehat{\mathcal{R}}}{\partial S_{1}} \cdot \mathbf{t}_{2} S_{3}-\frac{\partial \widehat{\mathcal{R}}}{\partial S_{1}} \cdot \mathbf{t}_{3} S_{2}\right) \mathbf{M}^{11}
\end{aligned}
$$

a.e. in $\Omega \times \mathcal{S} \times D$.

Now, we introduce 4 correctors which are the solutions to the following cell problems:

$$
\begin{aligned}
& \int_{D} a_{i j k l}(\mathbf{S}, \cdot)\left(\mathcal{E}_{D}\left(\tilde{\chi}_{1}\right)(\mathbf{S}, \cdot)+\mathbf{M}^{11}\right)_{i j}\left(\mathcal{E}_{D}(\widetilde{v})\right)_{k l} d S_{2} d S_{3}=0, \\
& \int_{D} a_{i j k l}(\mathbf{S}, \cdot)\left(\mathcal{E}_{D}\left(\tilde{\chi}_{2}\right)(\mathbf{S}, \cdot)+S_{2} \mathbf{M}^{13}-S_{3} \mathbf{M}^{12}\right)_{i j}\left(\mathcal{E}_{D}(\widetilde{v})\right)_{k l} d S_{2} d S_{3}=0, \\
& \int_{D} a_{i j k l}(\mathbf{S}, \cdot)\left(\mathcal{E}_{D}\left(\tilde{\chi}_{3}\right)(\mathbf{S}, \cdot)+S_{3} \mathbf{M}^{11}\right)_{i j}\left(\mathcal{E}_{D}(\widetilde{v})\right)_{k l} d S_{2} d S_{3}=0, \\
& \int_{D} a_{i j k l}(\mathbf{S}, \cdot)\left(\mathcal{E}_{D}\left(\tilde{\chi}_{4}\right)(\mathbf{S}, \cdot)-S_{2} \mathbf{M}^{11}\right)_{i j}\left(\mathcal{E}_{D}(\widetilde{v})\right)_{k l} d S_{2} d S_{3}=0, \\
& \quad \text { for a.e. } \mathbf{S} \text { in } \mathcal{S}, \quad \forall \widetilde{v} \in \mathcal{D}_{w} .
\end{aligned}
$$

Since $a_{i j k l}$ 's belong to $L^{\infty}(\mathcal{S} \times D)$, then $\tilde{\chi}_{q} \in L^{\infty}\left(\mathcal{S} ; \mathcal{D}_{w}\right), q \in\{1, \ldots, 4\}$.

Hence, we have

$$
\tilde{u}=\left(\left(e(\mathcal{U}) \mathbf{t}_{1}\right) \cdot \mathbf{t}_{1}+\frac{\partial \overline{\mathcal{U}}}{\partial \mathbf{S}} \cdot \mathbf{t}_{1}\right) \tilde{\chi}_{1}+\sum_{q=1}^{3} \frac{\partial \widetilde{\mathcal{R}}}{\partial \mathbf{S}} \cdot \mathbf{t}_{q} \tilde{\chi}_{q+1} \quad \text { a.e. in } \Omega \times \mathcal{S} \times D .
$$

\subsection{Expression of the Microscopic Fields $\overline{\mathcal{U}}, \widehat{\mathcal{U}}, \widehat{\mathcal{R}}$}

In this subsection we give the expression of the microscopic fields $\overline{\mathcal{U}}, \widehat{\mathcal{U}}, \widehat{\mathcal{R}}$ in terms of the macroscopic displacement $\mathcal{U}$. To this end, as before, we use the variational formulation (8.12).

Thus, taking $\mathcal{V}=0, \widetilde{v}=0$ in (8.12), then replacing $\tilde{u}$ by its expression, using the following equality: 


$$
\mathcal{E}_{D}(\widetilde{u})=\left(\left(e(\mathcal{U}) \mathbf{t}_{1}\right) \cdot \mathbf{t}_{1}+\frac{\partial \overline{\mathcal{U}}}{\partial \mathbf{S}} \cdot \mathbf{t}_{1}\right) \mathcal{E}_{D}\left(\tilde{\chi}_{1}\right)+\sum_{q=1}^{3} \frac{\partial \widehat{\mathcal{R}}}{\partial \mathbf{S}} \cdot \mathbf{t}_{q} \mathcal{E}_{D}\left(\widetilde{\chi}_{q+1}\right) \quad \text { a.e. in } \Omega \times \mathcal{S} \times D
$$

together with (9.1) give

$$
\begin{aligned}
& \int_{\mathcal{S}}\left(\int _ { D } a _ { i j k l } \left[\left(\left(e(\mathcal{U}) \mathbf{t}_{1}\right) \cdot \mathbf{t}_{1}+\frac{\partial \overline{\mathcal{U}}}{\partial \mathbf{S}} \cdot \mathbf{t}_{1}\right)\left(\mathcal{E}_{D}\left(\tilde{\chi}_{1}\right)+\mathbf{M}^{11}\right)\right.\right. \\
& +\frac{\partial \widehat{\mathcal{R}}}{\partial \mathbf{S}} \cdot \mathbf{t}_{1}\left(\mathcal{E}_{D}\left(\tilde{\chi}_{2}\right)+S_{2} \mathbf{M}^{13}-S_{3} \mathbf{M}^{12}\right) \\
& \left.\left.+\frac{\partial \widehat{\mathcal{R}}}{\partial \mathbf{S}} \cdot \mathbf{t}_{2}\left(\mathcal{E}_{D}\left(\tilde{\chi}_{3}\right)+S_{3} \mathbf{M}^{11}\right)+\frac{\partial \widehat{\mathcal{R}}}{\partial \mathbf{S}} \cdot \mathbf{t}_{3}\left(\mathcal{E}_{D}\left(\tilde{\chi}_{4}\right)-S_{2} \mathbf{M}^{11}\right)\right]_{i j}\left[\mathcal{E}_{\mathcal{S}}(\overline{\mathcal{V}}, \widehat{\mathcal{V}}, \widehat{\mathcal{B}})\right]_{k l} d S_{2} d S_{3}\right) d \mathbf{S} \\
& =\frac{4 \pi}{5} G \cdot \sum_{A \in \mathcal{K}} \widehat{\mathcal{B}}(A)+\frac{\pi}{1+\kappa} \mathbf{f} \cdot \int_{\mathcal{S}} \widehat{\mathcal{V}}(\mathbf{S}) d \mathbf{S}, \quad \text { a.e. in } \Omega, \quad \forall(\overline{\mathcal{V}}, \widehat{\mathcal{V}}, \widehat{\mathcal{B}}) \in \mathcal{D}_{E x} \times \mathcal{D}_{I n} .
\end{aligned}
$$

We write

$$
\mathcal{E}_{\mathcal{S}}(\overline{\mathcal{V}}, \widehat{\mathcal{V}}, \widehat{\mathcal{B}})=\left(\frac{\partial \overline{\mathcal{V}}}{\partial \mathbf{S}} \cdot \mathbf{t}_{1}\right) \mathbf{M}^{11}+\frac{\partial \widehat{\mathcal{B}}}{\partial \mathbf{S}} \cdot \mathbf{t}_{1}\left(S_{2} \mathbf{M}^{13}-S_{3} \mathbf{M}^{12}\right)+\left(\frac{\partial \widehat{\mathcal{B}}}{\partial \mathbf{S}} \cdot \mathbf{t}_{2} S_{3}-\frac{\partial \widehat{\mathcal{B}}}{\partial \mathbf{S}} \cdot \mathbf{t}_{3} S_{2}\right) \mathbf{M}^{11}
$$

and the variational problem (9.3) has the following form:

$$
\begin{aligned}
& \int_{\mathcal{S}} \mathfrak{A} \frac{\partial}{\partial \mathbf{S}}\left(\begin{array}{l}
\overline{\mathcal{U}} \\
\cdots \\
\widehat{\mathcal{R}}
\end{array}\right) \cdot \frac{\partial}{\partial \mathbf{S}}\left(\begin{array}{l}
\overline{\mathcal{V}} \\
\cdots \\
\widehat{\mathcal{B}}
\end{array}\right) d \mathbf{S}=-\int_{\mathcal{S}} \mathfrak{A}\left(\begin{array}{c}
\left(e(\mathcal{U}) \mathbf{t}_{1}\right) \cdot \mathbf{t}_{1} \\
\ldots \\
0
\end{array}\right) \cdot \frac{\partial}{\partial \mathbf{S}}\left(\begin{array}{l}
\overline{\mathcal{V}} \\
\cdots \\
\widehat{\mathcal{B}}
\end{array}\right) d \mathbf{S} \\
& +\frac{4 \pi}{5} G \cdot \sum_{A \in \mathcal{K}} \widehat{\mathcal{B}}(A)+\frac{\pi}{1+\kappa} \mathbf{f} \cdot \int_{\mathcal{S}} \widehat{\mathcal{V}}(\mathbf{S}) d \mathbf{S}, \quad \text { a.e. in } \Omega, \quad \forall(\overline{\mathcal{V}}, \widehat{\mathcal{V}}, \widehat{\mathcal{B}}) \in \mathcal{D}_{E x} \times \mathcal{D}_{I n}
\end{aligned}
$$

where the symmetric matrix $\mathfrak{A}$ belongs to $L^{\infty}(\mathcal{S})^{4 \times 4}$.

Here, the column $\frac{\partial}{\partial \mathbf{S}}\left(\begin{array}{c}\overline{\mathcal{V}} \\ \cdots \\ \widehat{\mathcal{B}}\end{array}\right)$ stands for the column $\left(\frac{\partial \overline{\mathcal{V}}}{\partial \mathbf{S}} \cdot \mathbf{t}_{1} \frac{\partial \widehat{\mathcal{B}}}{\partial \mathbf{S}} \cdot \mathbf{t}_{1} \frac{\partial \widehat{\mathcal{B}}}{\partial \mathbf{S}} \cdot \mathbf{t}_{2} \frac{\partial \widehat{\mathcal{B}}}{\partial \mathbf{S}} \cdot \mathbf{t}_{3}\right)^{T}$, while the column $\left(\begin{array}{c}\left(e(\mathcal{V}) \mathbf{t}_{1}\right) \cdot \mathbf{t}_{1} \\ 0\end{array}\right)$ stands for $\left(\left(e(\mathcal{V}) \mathbf{t}_{1}\right) \cdot \mathbf{t}_{1} \quad 0 \quad 000\right)^{T}$.

Matrix $\mathfrak{A}$ satisfies

$$
\begin{aligned}
& \forall \zeta \in \mathbb{R}^{4}, \\
& \begin{aligned}
\mathfrak{A} \zeta \cdot \zeta=\int_{D} a_{i j k l}[ & \zeta_{1}\left(\mathcal{E}_{D}\left(\widetilde{\chi}_{1}\right)+\mathbf{M}^{11}\right)+\zeta_{2}\left(\mathcal{E}_{D}\left(\tilde{\chi}_{2}\right)+S_{2} \mathbf{M}^{13}-S_{3} \mathbf{M}^{12}\right) \\
& \left.+\zeta_{3}\left(\mathcal{E}_{D}\left(\widetilde{\chi}_{3}\right)+S_{3} \mathbf{M}^{11}\right)+\zeta_{4}\left(\mathcal{E}_{D}\left(\widetilde{\chi}_{4}\right)-S_{2} \mathbf{M}^{11}\right)\right]_{i j} \\
\times & {\left[\zeta_{1}\left(\mathcal{E}_{D}\left(\tilde{\chi}_{1}\right)+\mathbf{M}^{11}\right)+\zeta_{2}\left(\mathcal{E}_{D}\left(\tilde{\chi}_{2}\right)+S_{2} \mathbf{M}^{13}-S_{3} \mathbf{M}^{12}\right)\right.} \\
& \left.+\zeta_{3}\left(\mathcal{E}_{D}\left(\tilde{\chi}_{3}\right)+S_{3} \mathbf{M}^{11}\right)+\zeta_{4}\left(\mathcal{E}_{D}\left(\tilde{\chi}_{4}\right)-S_{2} \mathbf{M}^{11}\right)\right]_{k l} d S_{2} d S_{3}
\end{aligned}
\end{aligned}
$$

a.e. in $\mathcal{S}$

since $\tilde{\chi}_{q}$ 's verify (9.2). 
At this step, the unfolded problem becomes

$$
\begin{aligned}
& \quad \int_{\Omega \times \mathcal{S}} \mathfrak{A}\left[\left(\begin{array}{c}
\left(e(\mathcal{U}) \mathbf{t}_{1}\right) \cdot \mathbf{t}_{1} \\
\cdots \\
0
\end{array}\right)+\frac{\partial}{\partial \mathbf{S}}\left(\begin{array}{l}
\overline{\mathcal{U}} \\
\cdots \\
\widehat{\mathcal{R}}
\end{array}\right)\right] \cdot\left[\left(\begin{array}{c}
\left(e(\mathcal{V}) \mathbf{t}_{1}\right) \cdot \mathbf{t}_{1} \\
\ldots \\
0
\end{array}\right)+\frac{\partial}{\partial \mathbf{S}}\left(\begin{array}{c}
\mathcal{V} \\
\cdots \\
\widehat{\mathcal{B}}
\end{array}\right)\right] d x d \mathbf{S} \\
& =\frac{4 \pi|\mathcal{K}|}{3} \int_{\Omega} F \cdot \mathcal{V} d x+\frac{4 \pi}{5} \int_{\Omega} G \cdot\left(\sum_{A \in \mathcal{K}} \widehat{\mathcal{B}}(\cdot, A)\right) d x \\
& \quad+\frac{\kappa|\mathcal{S}|}{1+\kappa} \int_{\Omega} \mathbf{f} \cdot \mathcal{V} d x+\frac{\pi}{1+\kappa} \int_{\Omega \times \mathcal{S}} \mathbf{f} \cdot \widehat{\mathcal{V}}(\cdot, \mathbf{S}) d x d \mathbf{S}, \\
& \forall \mathcal{V} \in H_{\Gamma}^{1}(\Omega)^{3}, \quad \forall(\overline{\mathcal{V}}, \widehat{\mathcal{V}}, \widehat{\mathcal{B}}) \in L^{2}\left(\Omega ; \mathcal{D}_{E x} \times \mathcal{D}_{I n}\right) .
\end{aligned}
$$

Now, we introduce 12 correctors

$$
\begin{gathered}
\chi^{i j} \doteq\left(\bar{\chi}^{i j}, \widehat{\chi}^{i j}, \widehat{\chi}^{i j}\right), \quad \chi^{q} \doteq\left(\bar{\chi}^{q}, \widehat{\chi}^{q}, \widehat{\chi}^{q}\right) \in \mathcal{D}_{E x} \times \mathcal{D}_{I n}, \\
(i, j) \in\{1,2,3\}^{2}, \quad q \in\{1, \ldots, 6\} .
\end{gathered}
$$

They are the solutions to the following variational problems:

$$
\begin{aligned}
& \chi^{i j} \doteq\left(\bar{\chi}^{i j}, \widehat{\chi}^{i j}, \widehat{\chi}^{i j}\right) \in \mathcal{D}_{E x} \times \mathcal{D}_{I n}, \\
& \int_{\mathcal{S}} \mathfrak{A} \frac{d}{d \mathbf{S}}\left(\begin{array}{l}
\bar{\chi}^{i j} \\
\widehat{\chi}^{i j}
\end{array}\right) \cdot \frac{d}{d \mathbf{S}}\left(\begin{array}{c}
\overline{\mathcal{V}} \\
\cdots \\
\widehat{\mathcal{B}}
\end{array}\right) d \mathbf{S}=-\int_{\mathcal{S}} \mathfrak{A}\left(\begin{array}{c}
\left(\mathbf{M}^{i j} \mathbf{t}_{1}\right) \cdot \mathbf{t}_{1} \\
0
\end{array}\right) \cdot \frac{d}{d \mathbf{S}}\left(\begin{array}{c}
\overline{\mathcal{V}} \\
\cdots \\
\widehat{\mathcal{B}}
\end{array}\right) d \mathbf{S} \\
& \forall(\overline{\mathcal{V}}, \widehat{\mathcal{V}}, \widehat{\mathcal{B}}) \in \mathcal{D}_{E x} \times \mathcal{D}_{I n}, \\
& \chi^{q} \doteq\left(\bar{\chi}^{q}, \widehat{\chi}^{q}, \widehat{\chi}^{q}\right) \in \mathcal{D}_{E x} \times \mathcal{D}_{I n}, \quad q \in\{1,2,3\}, \\
& \int_{\mathcal{S}} \mathfrak{A} \frac{d}{d \mathbf{S}}\left(\begin{array}{l}
\bar{\chi}^{q} \\
\cdots \\
\widehat{\chi}^{q}
\end{array}\right) \cdot \frac{d}{d \mathbf{S}}\left(\begin{array}{c}
\overline{\mathcal{V}} \\
\cdots \\
\widehat{\mathcal{B}}
\end{array}\right) d \mathbf{S}=\mathbf{e}_{q} \cdot \sum_{A \in \mathcal{K}} \widehat{\mathcal{B}}(A) \quad \forall(\overline{\mathcal{V}}, \widehat{\mathcal{V}}, \widehat{\mathcal{B}}) \in \mathcal{D}_{E x} \times \mathcal{D}_{I n}, \\
& \chi^{q+3} \doteq\left(\bar{\chi}^{q+3}, \widehat{\chi}^{q+3}, \widehat{\chi}^{q+3}\right) \in \mathcal{D}_{E x} \times \mathcal{D}_{I n}, \quad q \in\{1,2,3\}, \\
& \int_{\mathcal{S}} \mathfrak{A} \frac{d}{d \mathbf{S}}\left(\begin{array}{l}
\bar{\chi}^{q+3} \\
\widehat{\chi}^{q+3}
\end{array}\right) \cdot \frac{d}{d \mathbf{S}}\left(\begin{array}{l}
\mathcal{\mathcal { V }} \\
\cdots \\
\widehat{\mathcal{B}}
\end{array}\right) d \mathbf{S}=\mathbf{e}_{q} \cdot \int_{\mathcal{S}} \widehat{\mathcal{V}} d \mathbf{S}, \quad \forall(\overline{\mathcal{V}}, \widehat{\mathcal{V}}, \widehat{\mathcal{B}}) \in \mathcal{D}_{E x} \times \mathcal{D}_{I n},
\end{aligned}
$$

where $\mathbf{e}_{1}=\left(\begin{array}{lll}1 & 0 & 0\end{array}\right)^{T}, \mathbf{e}_{2}=\left(\begin{array}{lll}0 & 1 & 0\end{array}\right)^{T}$ and $\mathbf{e}_{3}=\left(\begin{array}{lll}0 & 0 & 1\end{array}\right)^{T}$. Note that $\chi^{i j}=\chi^{j i}$.

Hence, one has

$$
(\overline{\mathcal{U}}, \widehat{\mathcal{U}}, \widehat{\mathcal{R}})=\sum_{i, j=1}^{3} e_{i j}(\mathcal{U}) \chi^{i j}+\frac{4 \pi}{5} \sum_{q=1}^{3} G_{q} \chi^{q}+\frac{\pi}{1+\kappa} \sum_{q=1}^{3} \mathbf{f}_{q} \chi^{q+3},
$$

where $G=\sum_{q=1}^{3} G_{q} \mathbf{e}_{q}, \mathbf{f}=\sum_{q=1}^{3} \mathbf{f}_{q} \mathbf{e}_{q}$. 
In problem $(9.5)$, we replace $(\overline{\mathcal{U}}, \widehat{\mathcal{U}}, \widehat{\mathcal{R}})$ by $(9.7)$ and we choose $(\overline{\mathcal{V}}, \widehat{\mathcal{V}}, \widehat{\mathcal{B}})=(0,0,0)$. That gives

$$
\begin{array}{rl}
\int_{\Omega \times \mathcal{S}} & \mathfrak{A}\left[\left(\begin{array}{c}
\left(e(\mathcal{U}) \mathbf{t}_{1}\right) \cdot \mathbf{t}_{1} \\
\cdots \\
0
\end{array}\right)+\sum_{i, j=1}^{3} e_{i j}(\mathcal{U}) \frac{\partial}{\partial \mathbf{S}}\left(\begin{array}{l}
\bar{\chi}^{i j} \\
\cdots \\
\hat{\chi}^{i j}
\end{array}\right)\right] \cdot\left(\begin{array}{c}
\left(e(\mathcal{V}) \mathbf{t}_{1}\right) \cdot \mathbf{t}_{1} \\
\cdots \\
0
\end{array}\right) d x d \mathbf{S} \\
= & -\int_{\Omega}\left(\frac{4 \pi}{5} \sum_{q=1}^{3} G_{q}\left[\int_{\mathcal{S}} \mathfrak{A} \frac{\partial}{\partial \mathbf{S}}\left(\begin{array}{l}
\bar{\chi}^{q} \\
\cdots \\
\widehat{\chi}^{q}
\end{array}\right) \cdot\left(\begin{array}{c}
\left(e(\mathcal{V}) \mathbf{t}_{1}\right) \cdot \mathbf{t}_{1} \\
\ldots \\
0
\end{array}\right) d \mathbf{S}\right]\right. \\
& \left.+\frac{\pi}{1+\kappa} \sum_{q=1}^{3} \mathbf{f}_{q}\left[\int_{\mathcal{S}} \mathfrak{A} \frac{\partial}{\partial \mathbf{S}}\left(\begin{array}{c}
\bar{\chi}^{q+3} \\
\hat{\chi}^{q+3}
\end{array}\right) \cdot\left(\begin{array}{c}
\left(e(\mathcal{V}) \mathbf{t}_{1}\right) \cdot \mathbf{t}_{1} \\
\ldots \\
0
\end{array}\right) d \mathbf{S}\right]\right) d x \\
& +\frac{4 \pi|\mathcal{K}|}{3} \int_{\Omega} F \cdot \mathcal{V} d x+\frac{\kappa|\mathcal{S}|}{1+\kappa} \int_{\Omega} \mathbf{f} \cdot \mathcal{V} d x, \quad \forall \mathcal{V} \in H_{\Gamma}^{1}(\Omega)^{3} .
\end{array}
$$

Now, taking into account the definition of the corrector $\chi^{i j} \doteq\left(\bar{\chi}^{i j}, \widehat{\chi}^{i j}, \widehat{\chi}^{i j}\right)$, the left-hand side becomes

$$
\int_{\Omega} \mathfrak{B}^{\text {hom }}(e(\mathcal{U}), e(\mathcal{V})) d x
$$

where $\mathfrak{B}^{\text {hom }}$ is a symmetric bilinear form associated to the definite positive quadratic form

$$
\begin{aligned}
& \mathfrak{B}^{\text {hom }}(\zeta, \zeta) \\
& =\int_{\mathcal{S}} \mathfrak{A}\left[\left(\begin{array}{c}
\left(\zeta \mathbf{t}_{1}\right) \cdot \mathbf{t}_{1} \\
\cdots \\
0
\end{array}\right)+\sum_{i, j=1}^{3} \zeta_{i j} \frac{\partial}{\partial \mathbf{S}}\left(\begin{array}{l}
\bar{\chi}^{i j} \\
\cdots \\
\hat{\chi}^{i j}
\end{array}\right)\right] \cdot\left(\begin{array}{c}
\left(\zeta \mathbf{t}_{1}\right) \cdot \mathbf{t}_{1} \\
\cdots \\
0
\end{array}\right) d \mathbf{S} \\
& \quad=\int_{\mathcal{S}} \mathfrak{A}\left[\left(\begin{array}{c}
\left(\zeta \mathbf{t}_{1}\right) \cdot \mathbf{t}_{1} \\
\cdots \\
0
\end{array}\right)+\sum_{i, j=1}^{3} \zeta_{i j} \frac{\partial}{\partial \mathbf{S}}\left(\begin{array}{l}
\bar{\chi}^{i j} \\
\cdots \\
\hat{\chi}^{i j}
\end{array}\right)\right] \cdot\left[\left(\begin{array}{c}
\left(\zeta \mathbf{t}_{1}\right) \cdot \mathbf{t}_{1} \\
\cdots \\
0
\end{array}\right)+\sum_{i, j=1}^{3} \zeta_{i j} \frac{\partial}{\partial \mathbf{S}}\left(\begin{array}{l}
\bar{\chi}^{i j} \\
\cdots \\
\hat{\chi}^{i j}
\end{array}\right)\right] d \mathbf{S} \\
& =\mathfrak{b}_{i j k l}^{\text {hom }} \zeta_{i j} \zeta_{k l}
\end{aligned}
$$

for every $3 \times 3$ symmetric matrix $\zeta$.

Write $\zeta=\sum_{i, j=1}^{3} \zeta_{i j} \mathbf{M}^{i j}$. Hence,

$$
\mathfrak{b}_{i j k l}^{h o m}=\int_{\mathcal{S}} \mathfrak{A}\left[\left(\begin{array}{c}
\left(\mathbf{M}^{i j} \mathbf{t}_{1}\right) \cdot \mathbf{t}_{1} \\
\cdots \\
0
\end{array}\right)+\frac{\partial}{\partial \mathbf{S}}\left(\begin{array}{l}
\bar{\chi}^{i j} \\
\cdots \\
\widehat{\chi}^{i j}
\end{array}\right)\right] \cdot\left[\left(\begin{array}{c}
\left(\mathbf{M}^{k l} \mathbf{t}_{1}\right) \cdot \mathbf{t}_{1} \\
\cdots \\
0
\end{array}\right)+\frac{\partial}{\partial \mathbf{S}}\left(\begin{array}{l}
\bar{\chi}^{k l} \\
\cdots \\
\hat{\chi}^{k l}
\end{array}\right)\right] d \mathbf{S} .
$$

Now, we simplify the right-hand side of (9.8). Set

$$
\begin{gathered}
\mathfrak{c}_{i j q}^{\text {hom }}=\int_{\mathcal{S}} \mathfrak{A} \frac{\partial}{\partial \mathbf{S}}\left(\begin{array}{l}
\bar{\chi}^{q} \\
\cdots \\
\widehat{\chi}^{q}
\end{array}\right) \cdot\left[\left(\begin{array}{c}
\left(\mathbf{M}^{i j} \mathbf{t}_{1}\right) \cdot \mathbf{t}_{1} \\
\cdots \\
0
\end{array}\right)+\frac{\partial}{\partial \mathbf{S}}\left(\begin{array}{l}
\bar{\chi}^{i j} \\
\cdots \\
\widehat{\chi}^{i j}
\end{array}\right)\right] d \mathbf{S}, \\
(i, j, q) \in\{1,2,3\}^{2} \times\{1, \ldots, 6\} .
\end{gathered}
$$

Thus, the limit field $\mathcal{U} \in H_{\Gamma}^{1}(\Omega)^{3}$ is the solution to the homogenized problem

$$
\int_{\Omega} \mathfrak{b}_{i j k l}^{h o m} e_{i j}(\mathcal{U}) e_{k l}(\mathcal{V}) d x
$$




$$
\begin{aligned}
= & -\frac{4 \pi}{5} \sum_{q=1}^{3} \int_{\Omega} G_{q} \mathfrak{c}_{i j q}^{h o m} e_{i j}(\mathcal{V}) d x+\frac{\pi}{1+\kappa} \sum_{q=1}^{3} \int_{\Omega} \mathbf{f}_{q} \mathfrak{c}_{i j q+3}^{h o m} e_{i j}(\mathcal{V}) d x \\
& +\frac{4 \pi|\mathcal{K}|}{3} \int_{\Omega} F \cdot \mathcal{V} d x+\frac{\kappa|\mathcal{S}|}{1+\kappa} \int_{\Omega} \mathbf{f} \cdot \mathcal{V} d x, \quad \forall \mathcal{V} \in H_{\Gamma}^{1}(\Omega)^{3} .
\end{aligned}
$$

Lemma 20 The components of the homogenized elasticity tensor $\mathfrak{b}_{i j k l} \in \mathbb{R}$ satisfy the usual symmetry and positivity conditions

$-\mathfrak{b}_{i j k l}^{\text {hom }}=\mathfrak{b}_{j i k l}^{\text {hom }}=\mathfrak{b}_{\text {klij }}^{\text {hom }}$

- there exists $C_{0}^{*}>0$ such that for every $3 \times 3$ symmetric matrix, one has

$$
\mathfrak{B}^{h o m}(\zeta, \zeta)=\mathfrak{b}_{i j k l}^{h o m} \zeta_{i j} \zeta_{k l} \geq C_{0}^{*}|\zeta|^{2}
$$

Proof By definition of the $\mathfrak{b}_{i j k l}^{\text {hom }}$, s, the symmetry of matrices $M^{i j}=M^{j i}$ and correctors $\chi^{i j}=$ $\chi^{j i}$ we obtain the symmetries of the $\mathfrak{b}_{i j k l}^{\text {hom }}$ 's.

From equality (9.9), Lemma 27 and estimate (G.4) we have

$$
\begin{aligned}
& \mathfrak{B}^{\text {hom }}(\zeta, \zeta) \\
& \quad=\int_{\mathcal{S}} \mathfrak{A}\left[\left(\begin{array}{c}
\left(\zeta \mathbf{t}_{1}\right) \cdot \mathbf{t}_{1} \\
\cdots \\
0
\end{array}\right)+\sum_{i, j=1}^{3} \zeta_{i j} \frac{\partial}{\partial \mathbf{S}}\left(\begin{array}{l}
\bar{\chi}^{i j} \\
\cdots \\
\hat{\chi}^{i j}
\end{array}\right)\right] \cdot\left[\left(\begin{array}{c}
\left.\zeta \mathbf{t}_{1}\right) \cdot \mathbf{t}_{1} \\
\cdots \\
0
\end{array}\right)+\sum_{i, j=1}^{3} \zeta_{i j} \frac{\partial}{\partial \mathbf{S}}\left(\begin{array}{l}
\bar{\chi}^{i j} \\
\cdots \\
\hat{\chi}^{i j}
\end{array}\right)\right] d \mathbf{S} \\
& \quad \geq C_{0}^{\prime} \int_{\mathcal{S}}\left|\left(\begin{array}{c}
\left(\zeta \mathbf{t}_{1}\right) \cdot \mathbf{t}_{1} \\
\cdots \\
0
\end{array}\right)+\sum_{i, j=1}^{3} \zeta_{i j} \frac{\partial}{\partial \mathbf{S}}\left(\begin{array}{l}
\bar{\chi}^{i j} \\
\cdots \\
\hat{\chi}^{i j}
\end{array}\right)\right|^{2} d \mathbf{S} \geq C_{0}^{*}|\zeta|^{2} .
\end{aligned}
$$

Theorem 4 (The homogenized limit problem) The limit field $\mathcal{U} \in H_{\Gamma}^{1}(\Omega)^{3}$ is the unique solution to the homogenized problem

$$
\begin{aligned}
\int_{\Omega} \mathfrak{b}_{i j k l}^{h o m} e_{i j}(\mathcal{U}) e_{k l}(\mathcal{V}) d x & \\
= & -\frac{4 \pi}{5} \sum_{q=1}^{3} \int_{\Omega} G_{q} \mathfrak{c}_{i j q}^{h o m} e_{i j}(\mathcal{V}) d x+\frac{\pi}{1+\kappa} \sum_{q=1}^{3} \int_{\Omega} \mathbf{f}_{q} \mathfrak{c}_{i j q+3}^{h o m} e_{i j}(\mathcal{V}) d x \\
& +\frac{4 \pi|\mathcal{K}|}{3} \int_{\Omega} F \cdot \mathcal{V} d x+\frac{\kappa|\mathcal{S}|}{1+\kappa} \int_{\Omega} \mathbf{f} \cdot \mathcal{V} d x, \quad \forall \mathcal{V} \in H_{\Gamma}^{1}(\Omega)^{3},
\end{aligned}
$$

where the $\mathfrak{b}_{i j k l}^{\text {hom }}$ are given by (9.10) and the $\mathfrak{c}_{i j q}^{\text {hom }}$ by (9.11).

\section{The Case of an Isotropic and Homogeneous Material}

We consider an isotropic and homogeneous material for which the relation between the linearized strain tensor and the stress tensor is given as follows

$$
\sigma(u)=\lambda \operatorname{Tr}(e(u)) \mathbf{I}_{3}+2 \mu e(u),
$$


where $\mathbf{I}_{3}$ is the unit $3 \times 3$ matrix and $\lambda, \mu$ are the material Lamé constants.

The correctors $\tilde{\chi}_{q} \in L^{\infty}\left(\mathcal{S} ; \mathcal{D}_{w}\right), q \in\{1,2,3,4\}$, have the following form (see [13])

$$
\begin{array}{ll}
\tilde{\chi}_{1}\left(\cdot, S_{2}, S_{3}\right)=-v\left(S_{2} \mathbf{t}_{2}+S_{3} \mathbf{t}_{3}\right), & \tilde{\chi}_{2}\left(\cdot, S_{2}, S_{3}\right)=0, \\
\tilde{\chi}_{3}\left(\cdot, S_{2}, S_{3}\right)=v\left(-S_{2} S_{3} \mathbf{t}_{2}+\frac{S_{3}^{2}-S_{2}^{2}}{2} \mathbf{t}_{3}\right), & \tilde{\chi}_{4}\left(\cdot, S_{2}, S_{3}\right)=v\left(\frac{S_{2}^{2}-S_{3}^{2}}{2} \mathbf{t}_{2}+S_{2} S_{3} \mathbf{t}_{3}\right),
\end{array}
$$

where $v=\frac{\lambda}{2(\mu+\lambda)}$ is the Poisson coefficient.

Due to the symmetries of the elasticity coefficients and cross-sections, we have immediately

$$
\tilde{\chi}_{3}\left(\cdot, S_{2}, S_{3}\right)=-\tilde{\chi}_{4}\left(\cdot, S_{3}, S_{2}\right)
$$

Hence, we obtain

$$
\begin{aligned}
\tilde{u}=v\left[-\left(\left(e(\mathcal{U}) \mathbf{t}_{1}\right) \cdot \mathbf{t}_{1}+\right.\right. & \left.\frac{\partial \overline{\mathcal{U}}}{\partial \mathbf{S}} \cdot \mathbf{t}_{1}\right)\left(S_{2} \mathbf{t}_{2}+S_{3} \mathbf{t}_{3}\right)+\frac{\partial^{2} \widehat{\mathcal{U}}}{\partial \mathbf{S}^{2}} \cdot \mathbf{t}_{2}\left(\frac{S_{2}^{2}-S_{3}^{2}}{2} \mathbf{t}_{2}+S_{2} S_{3} \mathbf{t}_{3}\right) \\
& \left.+\frac{\partial^{2} \widehat{\mathcal{U}}}{\partial \mathbf{S}^{2}} \cdot \mathbf{t}_{3}\left(S_{2} S_{3} \mathbf{t}_{2}+\frac{S_{3}^{2}-S_{2}^{2}}{2} \mathbf{t}_{3}\right)\right] \quad \text { a.e. in } \Omega \times \mathcal{S} \times D .
\end{aligned}
$$

The matrix $\mathfrak{A}$ becomes

$$
\mathfrak{A}=\left(\begin{array}{cccc}
\pi E & 0 & 0 & 0 \\
0 & \frac{\pi}{2} \mu & 0 & 0 \\
0 & 0 & \frac{\pi}{4} E & 0 \\
0 & 0 & 0 & \frac{\pi}{4} E
\end{array}\right),
$$

where $E=\frac{\mu(3 \lambda+2 \mu)}{\lambda+\mu}$ is the Young's modulus.

The correctors $\chi^{i j} \doteq\left(\bar{\chi}^{i j}, \widehat{\chi}^{i j}, \widehat{\chi}^{i j}\right) \in L^{\infty}\left(\Omega ; \mathcal{D}_{E x} \times \mathcal{D}_{I n}\right),(i, j) \in\{1,2,3\}^{2}$.

These correctors are the solutions to the variational problems $(9.6)_{1}$. Hence, by virtue of (10.3), we have

$$
\begin{aligned}
& \int_{\mathcal{S}}\left(\mathfrak{a}_{1,1} \frac{d \bar{\chi}^{i j}}{d \mathbf{S}} \cdot \mathbf{t}_{1} \frac{d \overline{\mathcal{V}}}{d \mathbf{S}} \cdot \mathbf{t}_{1}+\sum_{q=1}^{3} \mathfrak{a}_{q+1, q+1} \frac{d \widehat{\chi}^{i j}}{d \mathbf{S}} \cdot \mathbf{t}_{q} \frac{d \widehat{\mathcal{B}}}{d \mathbf{S}} \cdot \mathbf{t}_{q}\right) d \mathbf{S} \\
& =-\int_{\mathcal{S}} \mathfrak{a}_{1,1}\left(\mathbf{M}^{i j} \mathbf{t}_{1}\right) \cdot \mathbf{t}_{1} \frac{d \overline{\mathcal{V}}}{d \mathbf{S}} \cdot \mathbf{t}_{1} d \mathbf{S} .
\end{aligned}
$$

Choosing the function $\left(0, \widehat{\chi}^{i j}, \widehat{\chi}^{i j}\right)$ as a test function we obtain

$$
\int_{\mathcal{S}} \sum_{q=1}^{3} \mathfrak{a}_{q+1, q+1} \frac{d \widehat{\chi}^{i j}}{d \mathbf{S}} \cdot \mathbf{t}_{q}^{\ell} \frac{d \widehat{\chi}^{i j}}{d \mathbf{S}} \cdot \mathbf{t}_{q}^{\ell} d \mathbf{S}=0 .
$$

Hence, for every $(i, j) \in\{1,2,3\}^{2}$ one has $\left(\widehat{\chi}^{i j}, \widehat{\chi}^{i j}\right)=(0,0)$.

Let $\ell$ be in $\{1, \ldots, m\}$ and $\phi \in H_{0}^{1}\left(\gamma_{\ell}\right)$. Consider the test function $\overline{\mathcal{V}} \in \mathcal{D}_{E x}$ defined by

$$
\overline{\mathcal{V}}= \begin{cases}\phi \mathbf{t}_{1}^{\ell} & \text { on } \gamma_{\ell}, \\ 0 & \text { on the other segments of } \mathcal{S} .\end{cases}
$$


That gives

$$
\int_{\gamma_{\ell}} \frac{d \bar{\chi}^{i j}}{d \mathbf{S}} \cdot \mathbf{t}_{1}^{\ell} \frac{d \phi}{d \mathbf{S}} d \mathbf{S}=-\int_{\gamma_{\ell}}\left(\mathbf{M}^{i j} \mathbf{t}_{1}^{\ell}\right) \cdot \mathbf{t}_{1}^{\ell} \frac{d \phi}{d \mathbf{S}} d \mathbf{S}
$$

and then

$$
\frac{d^{2} \bar{\chi}^{i j}}{d \mathbf{S}^{2}} \cdot \mathbf{t}_{1}^{\ell}=0 \quad \text { in } H^{-1}\left(\gamma_{\ell}\right)
$$

It means that $\bar{\chi}^{i j} \cdot \mathbf{t}_{1}$ is affine on every segment of $\mathcal{S}$. The function $\bar{\chi}^{i j}$ belongs to $\mathbf{U}_{\mathcal{S}}$. Set

$$
\mathbf{U}_{\mathcal{S}, p e r, 0} \doteq \mathbf{U}_{\mathcal{S}} \cap H_{p e r, 0}^{1}(\mathcal{S})^{3} .
$$

For every $(i, j) \in\{1,2,3\}^{2}$ one has

$$
\bar{\chi}^{i j} \in \mathbf{U}_{\mathcal{S}, p e r, 0} .
$$

Denote $\overline{\mathbf{M}}^{i j}$ the restriction to $\mathcal{S}$ of the linear field $x \in \mathbb{R}^{3} \longmapsto \mathbf{M}^{i j} x \in \mathbb{R}^{3}$. It belongs to $\mathbf{U}_{\mathcal{S}}$. Problem (10.4) becomes

$$
\int_{\mathcal{S}} \frac{d}{d \mathbf{S}}\left(\bar{\chi}^{i j}+\overline{\mathbf{M}}^{i j}\right) \cdot \mathbf{t}_{1} \frac{d \overline{\mathcal{V}}}{d \mathbf{S}} \cdot \mathbf{t}_{1} d \mathbf{S}=0, \quad \forall \overline{\mathcal{V}} \in \mathbf{U}_{\mathcal{S}, p e r, 0}
$$

The corrector $\bar{\chi}^{i j}$ is the projection on $\mathbf{U}_{\mathcal{S}, \text { per }, 0}$ of the field $\overline{\mathbf{M}}^{i j} \in \mathbf{U}_{\mathcal{S}}$ for the scalar product $<\cdot, \cdot\rangle_{1}$ (see (2.2) and Lemma 1).

The correctors: $\chi^{q} \doteq\left(\bar{\chi}^{q}, \widehat{\chi}^{q}, \widehat{\chi}^{q}\right) \in L^{\infty}\left(\Omega ; \mathcal{D}_{E x} \times \mathcal{D}_{I n}\right), q \in\{1,2,3\}$.

They are the solution to the following variational problems $(9.6)_{2}$. Hence, by virtue (10.3), we have

$$
\int_{\mathcal{S}}\left(\mathfrak{a}_{1,1} \frac{d \bar{\chi}^{q}}{d \mathbf{S}} \cdot \mathbf{t}_{1} \frac{d \overline{\mathcal{V}}}{d \mathbf{S}} \cdot \mathbf{t}_{1}+\sum_{i=1}^{3} \mathfrak{a}_{i+1, i+1} \frac{d \widehat{\chi}^{q}}{d \mathbf{S}} \cdot \mathbf{t}_{i} \frac{d \widehat{\mathcal{B}}}{d \mathbf{S}} \cdot \mathbf{t}_{i}\right) d \mathbf{S}=\mathbf{e}_{q} \cdot \sum_{A \in \mathcal{K}} \widehat{\mathcal{B}}(A) .
$$

Choosing the function $\left(\bar{\chi}^{q}, 0,0\right)$ as a test function we obtain

$$
\int_{\mathcal{S}} \frac{d \bar{\chi}^{q}}{d \mathbf{S}} \cdot \mathbf{t}_{1} \frac{d \bar{\chi}^{q}}{d \mathbf{S}} \cdot \mathbf{t}_{1} d \mathbf{S}=0
$$

Hence, for every $q \in\{1,2,3\}$ one has $\bar{\chi}^{q}=0$, since this function belongs to $\mathcal{D}_{E x}$.

Let $\ell$ be in $\{1, \ldots, m\}$ and $\phi_{1} \in H_{0}^{1}\left(\gamma_{\ell}\right), \phi_{2}, \phi_{3} \in H_{0}^{2}\left(\gamma_{\ell}\right)$. Consider the test function defined by

$$
\widehat{\mathcal{V}}=\left\{\begin{array}{l}
\phi_{2} \mathbf{t}_{2}^{\ell}+\phi_{3} \mathbf{t}_{3}^{\ell} \quad \text { on } \gamma_{\ell}, \\
0 \quad \text { on the other segments of } \mathcal{S},
\end{array} \quad \widehat{\mathcal{B}}=\left\{\begin{array}{l}
\phi_{1} \mathbf{t}_{1}^{\ell}-\frac{d \widehat{\mathcal{V}}}{d \mathbf{S}} \wedge \mathbf{t}_{1}^{\ell} \quad \text { on } \gamma_{\ell}, \\
0 \text { on the other segments of } \mathcal{S} .
\end{array}\right.\right.
$$

The couple $(\widehat{\mathcal{V}}, \widehat{\mathcal{B}})$ belongs to $\mathcal{D}_{\text {In }}$. Choosing this couple as a test function in (10.5) leads to

$$
\frac{d^{2} \widehat{\chi}^{q}}{d S_{1}^{2}} \cdot \mathbf{t}_{1}^{\ell}=0 \quad \text { in } H^{-1}\left(\gamma_{\ell}\right), \quad \frac{d^{3} \widehat{\chi}^{q}}{d S_{1}^{3}} \cdot \mathbf{t}_{2}^{\ell}=\frac{d^{3} \widehat{\chi}^{q}}{d S_{1}^{3}} \cdot \mathbf{t}_{3}^{\ell}=0 \quad \text { in } H^{-2}\left(\gamma_{\ell}\right) .
$$


Hence, for every $\ell \in\{1, \ldots, m\} \widehat{\chi}^{q} \cdot \mathbf{t}_{1}^{\ell}$ is an affine function on $\gamma_{\ell}$, while $\widehat{\chi}^{q} \cdot \mathbf{t}_{2}^{\ell}$ and $\widehat{\chi}^{q} \cdot \mathbf{t}_{3}^{\ell}$ are polynomial functions of degree less than 2 on $\gamma_{\ell}$. A straightforward calculation gives the restriction of $\widehat{\chi}^{q}$ to the segment $\gamma_{\ell}\left(S_{1} \in\left[0, l_{\ell}\right]\right)$

$$
\begin{aligned}
\widehat{\chi}^{q}\left(S_{1}\right)= & \widehat{\chi}^{q}(A)\left(1-\frac{S_{1}}{l_{\ell}}\right)+\widehat{\chi}^{q}(B) \frac{S_{1}}{l_{\ell}} \\
& -3\left(\left(\widehat{\chi}^{q}(A)+\widehat{\chi}^{q}(B)\right)-\left(\widehat{\chi}^{q}(A)+\widehat{\chi}^{q}(B)\right) \cdot \mathbf{t}_{1}^{\ell} \cdot \mathbf{t}_{1}^{\ell}\right) \frac{S_{1}}{l_{\ell}}\left(1-\frac{S_{1}}{l_{\ell}}\right)
\end{aligned}
$$

since $\int_{\gamma_{\ell}} \widehat{\boldsymbol{\chi}}^{q} \cdot \mathbf{t}_{2}^{\ell} d \mathbf{S}=\int_{\gamma_{\ell}} \widehat{\chi}^{q} \cdot \mathbf{t}_{3}^{\ell} d \mathbf{S}=0$. Then, integration gives

$$
\begin{aligned}
\widehat{\chi}^{q}\left(S_{1}\right) & =\widehat{\chi}^{q}(A) \wedge \mathbf{t}_{1}^{\ell} S_{1}\left(1-\frac{S_{1}}{2 l_{\ell}}\right)+\widehat{\chi}^{q}(B) \wedge \mathbf{t}_{1}^{\ell} \frac{S_{1}^{2}}{2 l_{\ell}} \\
& -3\left(\widehat{\chi}^{q}(A)+\widehat{\chi}^{q}(B)\right) \wedge \mathbf{t}_{1}^{\ell} \frac{S_{1}^{2}}{l_{\ell}}\left(\frac{1}{2}-\frac{S_{1}}{3 l_{\ell}}\right),
\end{aligned}
$$

since $\widehat{\chi}^{q}(A)=\widehat{\chi}^{q}(B)=0$.

The correctors: $\chi^{q+3} \doteq\left(\bar{\chi}^{q+3}, \widehat{\chi}^{q+3}, \widehat{\chi}^{q+3}\right) \in L^{\infty}\left(\Omega ; \mathcal{D}_{E x} \times \mathcal{D}_{I n}\right), q \in\{1,2,3\}$.

They are the solution to the variational problems (9.6) 3 . Hence by virtue (10.3) we have

$$
\int_{\mathcal{S}}\left(\mathfrak{a}_{1,1} \frac{d \bar{\chi}^{q+3}}{d \mathbf{S}} \cdot \mathbf{t}_{1} \frac{d \overline{\mathcal{V}}}{d \mathbf{S}} \cdot \mathbf{t}_{1}+\sum_{i=1}^{3} \mathfrak{a}_{i+1, i+1} \frac{d \widehat{\chi}^{q+3}}{d \mathbf{S}} \cdot \mathbf{t}_{i} \frac{d \widehat{\mathcal{B}}}{d \mathbf{S}} \cdot \mathbf{t}_{i}\right) d \mathbf{S}=\mathbf{e}_{q} \cdot \int_{\mathcal{S}} \widehat{\mathcal{V}} d \mathbf{S}
$$

As in the previous case, for every $q \in\{1,2,3\}$ one obtains $\bar{\chi}^{q+3}=0$.

Again, we consider the test function defined by (10.7). That leads to $(\ell \in\{1, \ldots, m\})$

$$
\begin{aligned}
& \frac{d^{2} \widehat{\chi}^{q+3}}{d S_{1}^{2}} \cdot \mathbf{t}_{1}^{\ell}=0 \text { in } H^{-1}\left(\gamma_{\ell}\right), \\
& \frac{d^{3} \widehat{\chi}^{q+3}}{d S_{1}^{3}} \cdot \mathbf{t}_{2}^{\ell}=\frac{4}{\pi E} \mathbf{e}_{q} \cdot \mathbf{t}_{2}^{\ell}, \quad \frac{d^{3} \widehat{\chi}^{q+3}}{d S_{1}^{3}} \cdot \mathbf{t}_{3}^{\ell}=\frac{4}{\pi E} \mathbf{e}_{q} \cdot \mathbf{t}_{3}^{\ell} \quad \text { in } H^{-2}\left(\gamma_{\ell}\right) .
\end{aligned}
$$

Hence, for every $\ell \in\{1, \ldots, m\}$, the restriction of $\widehat{\chi}^{q+3}$ to the segment $\gamma_{\ell}$ is $\left(S_{1} \in\left[0, l_{\ell}\right]\right)$

$$
\begin{aligned}
\widehat{\chi}^{q+3}\left(S_{1}\right) & =\widehat{\chi}^{q+3}(A)\left(1-\frac{S_{1}}{l_{\ell}}\right)+\widehat{\chi}^{q+3}(B) \frac{S_{1}}{l_{\ell}}-3\left(\left(\widehat{\chi}^{q+3}(A)+\widehat{\chi}^{q+3}(B)\right)\right. \\
& \left.-\left(\widehat{\chi}^{q+3}(A)+\widehat{\chi}^{q+3}(B)\right) \cdot \mathbf{t}_{1}^{\ell} \mathbf{t}_{1}^{\ell}\right) \frac{S_{1}}{l_{\ell}}\left(1-\frac{S_{1}}{l_{\ell}}\right) \\
& +\frac{2 l_{\ell}^{3}}{3 \pi E}\left(\mathbf{e}_{q}-\mathbf{e}_{q} \cdot \mathbf{t}_{1}^{\ell} \mathbf{t}_{1}^{\ell}\right) \frac{S_{1}}{l_{\ell}}\left(\frac{1}{2}-\frac{S_{1}}{l_{\ell}}\right)\left(1-\frac{S_{1}}{l_{\ell}}\right) .
\end{aligned}
$$


Then, integration gives

$$
\begin{aligned}
\widehat{\chi}^{q+3}\left(S_{1}\right) & =\widehat{\chi}^{q+3}(A) \wedge \mathbf{t}_{1}^{\ell} S_{1}\left(1-\frac{S_{1}}{2 l_{\ell}}\right)+\widehat{\chi}^{q+3}(B) \wedge \mathbf{t}_{1}^{\ell} \frac{S_{1}^{2}}{2 l_{\ell}} \\
& -3\left(\widehat{\chi}^{q+3}(A)+\widehat{\chi}^{q+3}(B)\right) \wedge \mathbf{t}_{1}^{\ell} \frac{S_{1}^{2}}{l_{\ell}}\left(\frac{1}{2}-\frac{S_{1}}{3 l_{\ell}}\right)-\frac{l_{\ell}^{4}}{6 \pi E} \frac{S_{1}^{2}}{l_{\ell}^{2}}\left(1-\frac{S_{1}}{l_{\ell}}\right)^{2} \mathbf{e}_{q} \wedge \mathbf{t}_{1}^{\ell} .
\end{aligned}
$$

The last step allows us to reduce the corrector problems $(9.6)_{2,3}$ to the algebraic equations with respect to the unknown vector of nodal values. Denote $\mathbf{E}_{q}$ the function belonging to $H_{p e r, 0}^{1}(\mathcal{S})^{3}$ and defined by $(\ell \in\{1, \ldots, m\})$

$$
\mathbf{E}_{q}\left(S_{1}\right)=\frac{l_{\ell}^{4}}{6 \pi E} \frac{S_{1}^{2}}{l_{\ell}^{2}}\left(1-\frac{S_{1}}{l_{\ell}}\right)^{2} \mathbf{e}_{q} \wedge \mathbf{t}_{1} \quad \text { on } \gamma_{\ell}, \quad\left(S_{1} \in\left[0, l_{\ell}\right]\right) .
$$

Set

$$
\begin{aligned}
\mathcal{P}_{\text {per }}(\mathcal{S})= & \left\{\widehat{\mathcal{B}} \in H_{\text {per }}^{1}(\mathcal{S})^{3} \mid \widehat{\mathcal{B}}\left(S_{1}\right)\right. \\
= & \widehat{\mathcal{B}}(A)\left(1-\frac{S_{1}}{l_{\ell}}\right)+\widehat{\mathcal{B}}(B) \frac{S_{1}}{l_{\ell}} \\
& -3\left[(\widehat{\mathcal{B}}(A)+\widehat{\mathcal{B}}(B))-\left((\widehat{\mathcal{B}}(A)+\widehat{\mathcal{B}}(B)) \cdot \mathbf{t}_{1}^{\ell}\right) \mathbf{t}_{1}^{\ell}\right] \frac{S_{1}}{l_{\ell}}\left(1-\frac{S_{1}}{l_{\ell}}\right), \\
& \text { on } \left.\quad \gamma_{\ell}=[A, B], \quad S_{1} \in\left[0, l_{\ell}\right], \quad \ell \in\{1, \ldots, m\}\right\} .
\end{aligned}
$$

So $\widehat{\chi}^{q},(q \in\{1,2,3\})$, belongs to $\mathcal{P}_{p e r}(\mathcal{S})$ and solves the discrete problem

$$
\begin{aligned}
& \widehat{\chi}^{q} \in \mathcal{P}_{p e r}(\mathcal{S}), \quad q \in\{1,2,3\} \\
& \int_{\mathcal{S}}\left(\sum_{i=1}^{3} \mathfrak{a}_{i+1, i+1} \frac{d \widehat{\chi}^{q}}{d \mathbf{S}} \cdot \mathbf{t}_{i} \frac{d \widehat{\mathcal{B}}}{d \mathbf{S}} \cdot \mathbf{t}_{i}\right) d \mathbf{S}=\mathbf{e}_{q} \cdot \sum_{A \in \mathcal{K}} \widehat{\mathcal{B}}(A), \quad \forall \mathcal{B} \in \mathcal{P}_{p e r}(\mathcal{S}) .
\end{aligned}
$$

Similarly $\widehat{\chi}^{q+3}+\frac{d \mathbf{E}_{q}}{d \mathbf{S}} \wedge \mathbf{t}_{1},(q \in\{1,2,3\})$, belongs to $\mathcal{P}_{\text {per }}(\mathcal{S})$ and solves the discrete problem

$$
\begin{aligned}
\widehat{\chi}^{q+3}+\frac{d \mathbf{E}_{q}}{d \mathbf{S}} \wedge \mathbf{t}_{1} \in \mathcal{P}_{p e r}(\mathcal{S}), \quad q \in\{1,2,3\}, \\
\int_{\mathcal{S}} \sum_{i=1}^{3} \mathfrak{a}_{i+1, i+1} \frac{d}{d \mathbf{S}}\left(\widehat{\chi}^{q+3}+\frac{d \mathbf{E}_{q}}{d \mathbf{S}} \wedge \mathbf{t}_{1}\right) \cdot \mathbf{t}_{i} \frac{d \widehat{\mathcal{B}}}{d \mathbf{S}} \cdot \mathbf{t}_{i} d \mathbf{S}=\int_{\mathcal{S}} \mathbf{e}_{q} \cdot \widehat{\mathcal{V}} d \mathbf{S} \\
\quad+\frac{\pi E}{4} \int_{\mathcal{S}}\left(\frac{d^{2} \mathbf{E}_{q}}{d \mathbf{S}^{2}} \wedge \mathbf{t}_{1}\right) \cdot \frac{d \widehat{\mathcal{B}}}{d \mathbf{S}} d \mathbf{S}, \quad \forall \mathcal{B} \in \mathcal{P}_{p e r}(\mathcal{S}) .
\end{aligned}
$$


One has

$$
\begin{aligned}
& \frac{\pi E}{4} \int_{\mathcal{S}}\left(\frac{d^{2} \mathbf{E}_{q}}{d \mathbf{S}^{2}} \wedge \mathbf{t}_{1}\right) \cdot \frac{d \widehat{\mathcal{B}}}{d \mathbf{S}} d \mathbf{S}=\sum_{\ell=1}^{m} \frac{l_{\ell}^{4}}{24} \int_{\gamma_{\ell}} \frac{d^{2}}{d S_{1}^{2}}\left(\frac{S_{1}^{2}}{l_{\ell}^{2}}\left(1-\frac{S_{1}}{l_{\ell}}\right)^{2}\right)\left(\mathbf{e}_{q} \wedge \mathbf{t}_{1}\right) \cdot \frac{d \widehat{\mathcal{B}}}{d \mathbf{S}} d \mathbf{S} \\
& =\sum_{\ell=1}^{m} \frac{l_{\ell}^{2}}{12}\left(\mathbf{e}_{q} \wedge \mathbf{t}_{1}\right) \cdot(\widehat{\mathcal{B}}(\mathbf{B})-\widehat{\mathcal{B}}(\mathbf{A}))-\sum_{\ell=1}^{m} \frac{l_{\ell}^{4}}{24} \int_{\gamma_{\ell}} \frac{d^{3}}{d S_{1}^{3}}\left(\frac{S_{1}^{2}}{l_{\ell}^{2}}\left(1-\frac{S_{1}}{l_{\ell}}\right)^{2}\right)\left(\mathbf{e}_{q} \wedge \mathbf{t}_{1}\right) \cdot \widehat{\mathcal{B}} d \mathbf{S} \\
& =\sum_{\ell=1}^{m} \frac{l_{\ell}^{2}}{12}\left(\mathbf{e}_{q} \wedge \mathbf{t}_{1}\right) \cdot(\widehat{\mathcal{B}}(\mathbf{B})-\widehat{\mathcal{B}}(\mathbf{A}))-\sum_{\ell=1}^{m} \int_{\gamma_{\ell}}\left(S_{1}-\frac{l_{\ell}}{2}\right)\left(\mathbf{e}_{q} \wedge \mathbf{t}_{1}\right) \cdot \widehat{\mathcal{B}} d \mathbf{S}
\end{aligned}
$$

and

$$
\begin{aligned}
\int_{\mathcal{S}} \mathbf{e}_{q} \cdot \widehat{\mathcal{V}} d \mathbf{S} & =\sum_{\ell=1}^{m} \int_{\gamma_{\ell}} \mathbf{e}_{q} \cdot \widehat{\mathcal{V}} d \mathbf{S}=-\sum_{\ell=1}^{m} \int_{\gamma_{\ell}}\left(S_{1}-\frac{l_{\ell}}{2}\right) \mathbf{e}_{q} \cdot \frac{d \widehat{\mathcal{V}}}{d \mathbf{S}} d \mathbf{S} \\
& =-\sum_{\ell=1}^{m} \int_{\gamma_{\ell}}\left(S_{1}-\frac{l_{\ell}}{2}\right) \mathbf{e}_{q} \cdot\left(\widehat{\mathcal{B}} \wedge \mathbf{t}_{1}\right) d \mathbf{S} \\
& =\sum_{\ell=1}^{m} \int_{\gamma_{\ell}}\left(S_{1}-\frac{l_{\ell}}{2}\right)\left(\mathbf{e}_{q} \wedge \mathbf{t}_{1}\right) \cdot \widehat{\mathcal{B}} d \mathbf{S} .
\end{aligned}
$$

Hence $\widehat{\chi}^{q+3}+\frac{d \mathbf{E}_{q}}{d \mathbf{S}} \wedge \mathbf{t}_{1},(q \in\{1,2,3\})$ are solutions of the discrete problem

$$
\begin{aligned}
& \widehat{\chi}^{q+3}+\frac{d \mathbf{E}_{q}}{d \mathbf{S}} \wedge \mathbf{t}_{1} \in \mathcal{P}_{\text {per }}(\mathcal{S}), \quad q \in\{1,2,3\} \\
& \int_{\mathcal{S}} \sum_{i=1}^{3} \mathfrak{a}_{i+1, i+1} \frac{d}{d \mathbf{S}}\left(\widehat{\chi}^{q+3}+\frac{d \mathbf{E}_{q}}{d \mathbf{S}} \wedge \mathbf{t}_{1}\right) \cdot \mathbf{t}_{i} \frac{d \widehat{\mathcal{B}}}{d \mathbf{S}} \cdot \mathbf{t}_{i} d \mathbf{S}=-\sum_{\ell=1}^{m} \frac{l_{\ell}^{2}}{12}\left(\int_{\gamma \ell} \frac{d \widehat{\mathcal{B}}}{d \mathbf{S}} \wedge \mathbf{t}_{1} d \mathbf{S}\right) \cdot \mathbf{e}_{q} \\
& \forall \mathcal{B} \in \mathcal{P}_{\text {per }}(\mathcal{S}) .
\end{aligned}
$$

\section{Conclusion}

We conclude, that for our $\varepsilon$-periodic $r$-thin structure, the solution to the linearized elasticity problem (5.7) (in the strong), or (5.8) (in the weak/variational form) can be reconstructed in the following form:

$$
\begin{aligned}
u_{\varepsilon}(x) \approx & \frac{\varepsilon}{r} \mathcal{U}(x)+\frac{4 \pi}{5} \frac{\varepsilon^{3}}{r^{2}} \sum_{q=1}^{3} G_{q}(x) \widehat{\chi}^{q}\left(\left\{\frac{x}{\varepsilon}\right\}_{\mid \mathcal{S}}\right) \\
& +\frac{\varepsilon^{5}}{r^{2}\left(\varepsilon^{2}+r\right)} \pi \sum_{q=1}^{3} \mathbf{f}_{q}(x) \widehat{\chi}^{q+3}\left(\left\{\frac{x}{\varepsilon}\right\}_{\mid \mathcal{S}}\right)+O\left(\frac{\varepsilon^{2}}{r}\right),
\end{aligned}
$$

for a.e. $x \in \mathcal{S}_{\varepsilon, r}$. 

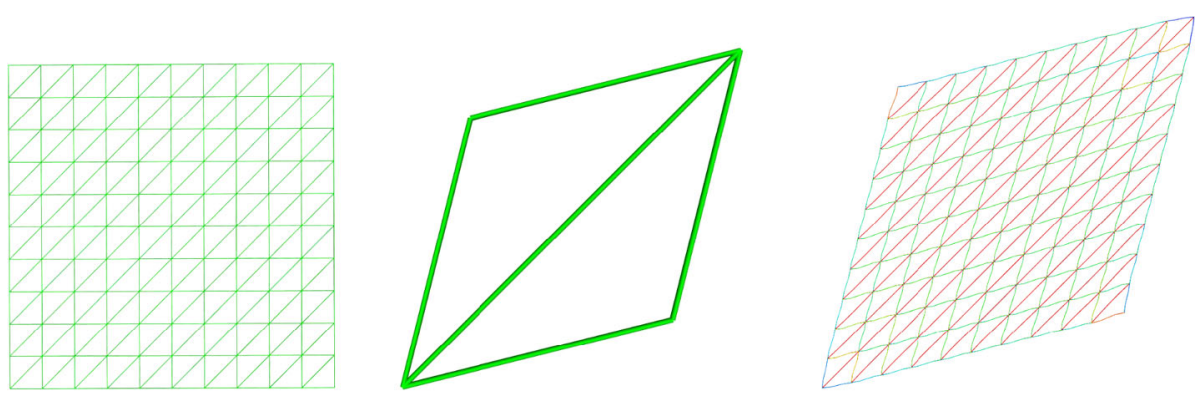

Fig. 4 Periodic stable 2D structure under in-plane loading. Solution of corrector problem $\chi^{12}$ and approximation of the solution for $\mathcal{U}\left(x_{1}, x_{2}\right)=x_{2} \mathbf{e}_{1}+x_{1} \mathbf{e}_{2}$

From Proposition 2 we have

$$
\begin{aligned}
& e_{s}\left(u_{\varepsilon}\right) \approx \frac{\varepsilon}{r}\left(\mathcal{E}(\mathcal{U})+\mathcal{E}_{S}(\overline{\mathcal{U}}, \widehat{\mathcal{U}}, \widehat{\mathcal{R}})+\mathcal{E}_{D}(\widetilde{u})\right) . \\
& \mathcal{E}(\mathcal{U}) \doteq\left(\begin{array}{ccc}
\left(e(\mathcal{U}) \mathbf{t}_{1}\right) \cdot \mathbf{t}_{1} & 0 & 0 \\
0 & 0 & 0 \\
0 & 0 & 0
\end{array}\right) \\
& \mathcal{E}_{S}(\overline{\mathcal{U}}, \widehat{\mathcal{U}}, \widehat{\mathcal{R}}) \doteq\left(\begin{array}{ccc}
\frac{\partial \overline{\mathcal{U}}}{\partial \mathbf{S}} \cdot \mathbf{t}_{1}-\frac{\partial^{2} \widehat{\mathcal{U}}}{\partial \mathbf{S}^{2}} \cdot\left(S_{2} \mathbf{t}_{2}+S_{3} \mathbf{t}_{3}\right) & * & * \\
-\frac{S_{3}}{2} \frac{\partial \mathcal{\mathcal { R }}}{\partial \mathbf{S}} \cdot \mathbf{t}_{1} & 0 & 0 \\
\frac{S_{2}}{2} \frac{\partial \mathcal{R}}{\partial \mathbf{S}} \cdot \mathbf{t}_{1} & 0 & 0
\end{array}\right) \text {, } \\
& \mathcal{E}_{D}(\widetilde{u}) \doteq\left(\begin{array}{ccc}
0 & \frac{1}{2} \frac{\partial \widetilde{u}}{\partial S_{2}} \cdot \mathbf{t}_{1} & \frac{1}{2} \frac{\partial \widetilde{u}}{\partial S_{3}} \cdot \mathbf{t}_{1} \\
* & \frac{\partial \widetilde{u}}{\partial S_{2}} \cdot \mathbf{t}_{2} & \frac{1}{2} \frac{\partial \widetilde{u}}{\partial S_{3}} \cdot \mathbf{t}_{2}+\frac{1}{2} \frac{\partial \widetilde{u}}{\partial S_{2}} \cdot \mathbf{t}_{3} \\
* & * & \frac{\partial \widetilde{u}}{\partial S_{3}} \cdot \mathbf{t}_{3}
\end{array}\right) \quad \text { a.e. in } \Omega \times \mathcal{S} \times D,
\end{aligned}
$$

where

$(\overline{\mathcal{U}}, \widehat{\mathcal{U}}, \widehat{\mathcal{R}})=\sum_{i, j=1}^{3} e_{i j}(\mathcal{U})\left(\bar{\chi}^{i j}, 0,0\right)+\frac{4 \pi}{5} \sum_{q=1}^{3} G_{q}\left(0, \widehat{\chi}^{q}, \widehat{\chi}^{q}\right)+\frac{\pi}{1+\kappa} \sum_{q=1}^{3} \mathbf{f}_{q}\left(0, \widehat{\chi}^{q+3}, \widehat{\chi}^{q+3}\right)$

and

$$
\widetilde{u}=v\left[\frac{\partial^{2} \widehat{\mathcal{U}}}{\partial \mathbf{S}^{2}} \cdot \mathbf{t}_{2}\left(\frac{S_{2}^{2}-S_{3}^{2}}{2} \mathbf{t}_{2}+S_{2} S_{3} \mathbf{t}_{3}\right)+\frac{\partial^{2} \widehat{\mathcal{U}}}{\partial \mathbf{S}^{2}} \cdot \mathbf{t}_{3}\left(S_{2} S_{3} \mathbf{t}_{2}+\frac{S_{3}^{2}-S_{2}^{2}}{2} \mathbf{t}_{3}\right)\right] .
$$

It is illustrated on Fig. 4. The strain tensor in the global coordinates can be obtained using (5.3). Then, we can reconstruct the local stress field for $\mathcal{P}_{\varepsilon \ell, r}^{\xi}$ beam as follows

$$
\sigma_{s}\left(u_{\varepsilon}\right) \approx \frac{\varepsilon}{r}\left(\begin{array}{ccc}
E\left(\left(e(\mathcal{U}) \mathbf{t}_{1}+\frac{\partial \overline{\mathcal{U}}}{\partial \mathbf{S}}\right) \cdot \mathbf{t}_{1}-\frac{\partial^{2} \widehat{\mathcal{U}}}{\partial \mathbf{S}^{2}} \cdot\left(S_{2} \mathbf{t}_{2}+S_{3} \mathbf{t}_{3}\right)\right) & * & * \\
-S_{3} \mu \frac{\partial \widehat{\mathcal{R}}}{\partial \mathbf{S}} \cdot \mathbf{t}_{1} & 0 & * \\
S_{2} \mu \frac{\partial \mathcal{\mathcal { R }}}{\partial \mathbf{S}} \cdot \mathbf{t}_{1} & 0 & 0
\end{array}\right)
$$

a.e. in $\Omega \times \mathcal{S} \times D$. 
Funding Note Open Access funding enabled and organized by Projekt DEAL.

Publisher's Note Springer Nature remains neutral with regard to jurisdictional claims in published maps and institutional affiliations.

Open Access This article is licensed under a Creative Commons Attribution 4.0 International License, which permits use, sharing, adaptation, distribution and reproduction in any medium or format, as long as you give appropriate credit to the original author(s) and the source, provide a link to the Creative Commons licence, and indicate if changes were made. The images or other third party material in this article are included in the article's Creative Commons licence, unless indicated otherwise in a credit line to the material. If material is not included in the article's Creative Commons licence and your intended use is not permitted by statutory regulation or exceeds the permitted use, you will need to obtain permission directly from the copyright holder. To view a copy of this licence, visit http://creativecommons.org/licenses/by/4.0/.

\section{Appendix A: Proof of Proposition 2}

Lemma 21 Let $\mathcal{S}$ be a 3-PSS. For every $u$ in $\mathbf{V}_{\varepsilon, r}$, one has

$$
\begin{aligned}
\|\mathcal{U}\|_{L^{2}\left(\Omega_{\varepsilon}^{\prime i n t}\right)} & \leq C \frac{\varepsilon}{r}\|e(u)\|_{L^{2}\left(\mathcal{S}_{\varepsilon, r}\right)}, \quad\|\nabla \mathcal{U}\|_{L^{2}\left(\Omega_{\varepsilon}^{\prime i n t}\right)} \leq C \frac{\varepsilon}{r}\|e(u)\|_{L^{2}\left(\mathcal{S}_{\varepsilon, r}\right)}, \\
\|\mathcal{R}\|_{L^{2}\left(\Omega_{\varepsilon}^{\prime i n t}\right)} & \leq C \frac{\varepsilon}{r}\|e(u)\|_{L^{2}\left(\mathcal{S}_{\varepsilon, r}\right)}, \quad\|\nabla \mathcal{R}\|_{L^{2}\left(\Omega_{\varepsilon}^{\prime i n t}\right)} \leq C \frac{1}{r}\|e(u)\|_{L^{2}\left(\mathcal{S}_{\varepsilon, r}\right)} .
\end{aligned}
$$

Proof Since $u$ belongs to $\mathbf{V}_{\varepsilon, r}$, by definition, it is equal to 0 in $\mathcal{S}_{\varepsilon, r}^{\prime} \backslash \overline{\mathcal{S}_{\varepsilon, r}}$. Then, there exists a rigid displacement $\mathbf{r}^{\prime}(x)=\mathbf{a}^{\prime}+\mathbf{b}^{\prime} \wedge x,\left(\mathbf{a}^{\prime}, \mathbf{b}^{\prime}\right) \in \mathbb{R}^{3} \times \mathbb{R}^{3}$ such that (using (5.17) with $\Omega^{\prime}$ instead of $\Omega$ )

$$
\left\|\mathcal{U}-\mathbf{r}^{\prime}\right\|_{H^{1}\left(\Omega_{\varepsilon}^{\prime i n t}\right)} \leq C \frac{\varepsilon}{r}\|e(u)\|_{L^{2}\left(\mathcal{S}_{\varepsilon, r}\right)} .
$$

Let $\mathcal{O}$ be an open set satisfying $\mathcal{O}$ strictly included in $\Omega^{\prime} \backslash \bar{\Omega}$.

If $\varepsilon$ is small enough then $\mathcal{O}_{\varepsilon}=\left\{x \in \mathbb{R}^{3} \mid \operatorname{dist}(x, \partial \mathcal{O})<2 \sqrt{3} \varepsilon\right\} \subset \Omega_{\varepsilon}^{\prime i n t} \backslash \overline{\Omega_{\varepsilon}^{i n t}}$. As a consequence $\mathcal{U}=\mathcal{R}=0$ a.e. in $\mathcal{O}$. Hence,

$$
\begin{gathered}
\left\|\mathbf{r}^{\prime}\right\|_{H^{1}(\mathcal{O})} \leq\left\|\mathcal{U}-\mathbf{r}^{\prime}\right\|_{H^{1}\left(\Omega_{\varepsilon}^{\prime i n t}\right)} \leq C \frac{\varepsilon}{r}\|e(u)\|_{L^{2}\left(\mathcal{S}_{\varepsilon, r}\right)} \quad \Longrightarrow \\
\left|\mathbf{a}^{\prime}\right|+\left|\mathbf{b}^{\prime}\right| \leq C\left\|\mathbf{r}^{\prime}\right\|_{H^{1}(\mathcal{O})} \leq C \frac{\varepsilon}{r}\|e(u)\|_{L^{2}\left(\mathcal{S}_{\varepsilon, r}\right)} .
\end{gathered}
$$

The constants do not depend on $\varepsilon$ and $r$. Therefore,

$$
\left\|\mathbf{r}^{\prime}\right\|_{H^{1}\left(\Omega_{\varepsilon}^{\prime i n t}\right)} \leq C_{0}\left(\left|\mathbf{a}^{\prime}\right|+\left|\mathbf{b}^{\prime}\right|\right) \leq C \frac{\varepsilon}{r}\|e(u)\|_{L^{2}\left(\mathcal{S}_{\varepsilon, r}\right)},
$$

where the constant $C_{0}$ only depends on the volume and diameter of $\Omega^{\prime}$. Finally,

$$
\|\mathcal{U}\|_{H^{1}\left(\Omega_{\varepsilon}^{i n t}\right)} \leq\|\mathcal{U}\|_{H^{1}\left(\Omega_{\varepsilon}^{\prime i n t}\right)} \leq\left\|\mathcal{U}-\mathbf{r}^{\prime}\right\|_{H^{1}\left(\Omega_{\varepsilon}^{\prime i n t}\right)}+\left\|\mathbf{r}^{\prime}\right\|_{H^{1}\left(\Omega_{\varepsilon}^{\prime i n t}\right)} \leq C \frac{\varepsilon}{r}\|e(u)\|_{L^{2}\left(\mathcal{S}_{\varepsilon, r}\right)}
$$

and (A.1) $)_{1,2}$ are proved. Estimates (A.1) $)_{3,4}$ follow from (A.1) $)_{1,2}$ and (5.16) 1,2 . 
Lemma 22 Let $\mathcal{S}$ be a 3-PSS. One has (see (5.14) for $\mathbf{a}$ and $\mathbf{b})$

$$
\begin{array}{ll}
\|\mathbf{a}\|_{L^{2}\left(\Omega_{\varepsilon}\right)}+\|\mathbf{b}\|_{L^{2}\left(\Omega_{\varepsilon}\right)} \leq C \frac{\varepsilon}{r}\|e(u)\|_{L^{2}\left(\mathcal{S}_{\varepsilon, r}\right)}, & \\
\|\mathcal{U}-\mathbf{a}\|_{L^{2}\left(\Omega_{\varepsilon}^{i n t}\right)} \leq C \frac{\varepsilon^{2}}{r}\|e(u)\|_{L^{2}\left(\mathcal{S}_{\varepsilon, r}\right)}, & \|\boldsymbol{\mathcal { R }}-\mathbf{b}\|_{L^{2}\left(\Omega_{\varepsilon}^{i n t}\right)} \leq \frac{C \varepsilon}{r}\|e(u)\|_{L^{2}\left(\mathcal{S}_{\varepsilon, r}\right)}, \\
\left\|\mathcal{U}^{h}-\mathbf{a}\right\|_{L^{2}\left(\mathcal{S}_{\varepsilon}\right)} \leq C \frac{\varepsilon}{r}\|e(u)\|_{L^{2}\left(\mathcal{S}_{\varepsilon, r}\right)}, & \left\|\frac{d \mathcal{U}^{h}}{d \mathbf{s}}-\mathbf{b} \wedge \mathbf{t}_{1}\right\|_{L^{2}\left(\mathcal{S}_{\varepsilon}\right)} \leq \frac{C}{r}\|e(u)\|_{L^{2}\left(\mathcal{S}_{\varepsilon, r}\right)}, \\
\left\|\mathcal{U}^{h}\right\|_{L^{2}\left(\mathcal{S}_{\varepsilon}\right)} \leq C \frac{1}{r}\|e(u)\|_{L^{2}\left(\mathcal{S}_{\varepsilon, r}\right)}, & \left\|\frac{d \mathcal{U}^{h}}{d \mathbf{s}}\right\|_{L^{2}\left(\mathcal{S}_{\varepsilon}\right)} \leq \frac{C}{r}\|e(u)\|_{L^{2}\left(\mathcal{S}_{\varepsilon, r}\right)} .
\end{array}
$$

Proof From estimates $(5.13)_{1},(5.15),(\text { A.1 })_{3}$ and the definition of $\mathcal{R}$ we obtain

$$
\sum_{\xi \in \Xi_{\varepsilon}}|\mathbf{b}(\varepsilon \xi)|^{2} \varepsilon^{3} \leq C \frac{\varepsilon^{2}}{r^{2}}\|e(u)\|_{L^{2}\left(\mathcal{S}_{\varepsilon, r}\right)}^{2} .
$$

Then, from the above estimate and $(5.13)_{2}$ we obtain

$$
\sum_{i=1}^{3} \sum_{\xi \in \Xi_{\varepsilon, i}}\left|\mathbf{a}\left(\varepsilon \xi+\varepsilon \mathbf{e}_{i}\right)-\mathbf{a}(\varepsilon \xi)\right|^{2} \varepsilon^{3} \leq C \frac{\varepsilon^{4}}{r^{2}}\|e(u)\|_{L^{2}\left(\mathcal{S}_{\varepsilon, r}\right)}^{2},
$$

which in turn with (5.15), (A.1) 1 and the definition of $\mathcal{U}$ lead to

$$
\sum_{\xi \in \Xi_{\varepsilon}}|\mathbf{a}(\varepsilon \xi)|^{2} \varepsilon^{3} \leq C \frac{\varepsilon^{2}}{r^{2}}\|e(u)\|_{L^{2}\left(\mathcal{S}_{\varepsilon, r}\right)}^{2} .
$$

Hence we have (A.2) $)_{1}$. Estimate (A.2) $)_{2}$ is the consequences of (A.2) $)_{1},($ A.4) and the definition of $\mathcal{U}$ while (A.2) $)_{3}$ follows from (5.13) ${ }_{1}$ and the definition of $\mathcal{R}$.

Estimate $(5.11)_{1}$ yields

$$
\left\|\mathcal{U}^{h}-\mathbf{a}(\varepsilon \xi)\right\|_{L^{2}(\varepsilon(\xi+\mathcal{S}))}^{2} \leq \frac{C \varepsilon^{2}}{r^{2}}\|e(u)\|_{L^{2}\left(\varepsilon\left(\xi+\mathcal{S}_{r / \varepsilon}\right)\right)}^{2}+C|\mathbf{b}(\varepsilon \xi)|^{2} \varepsilon^{3} .
$$

Summing up over all $\xi \in \Xi_{\varepsilon}$ the above inequality, using (5.11) 2 and applying (A.3) give (A.2) 4,5 .

Inequalities (A.2) 6,7 are the immediate consequences of (A.2) $)_{1,4,5}$.

Proof of Proposition 2 Since $\mathcal{U}=\mathcal{U}^{h}+\overline{\mathcal{U}}$ we have

$$
\|\mathcal{U}\|_{L^{2}\left(\mathcal{S}_{\varepsilon}\right)} \leq\left\|\mathcal{U}^{h}\right\|_{L^{2}\left(\mathcal{S}_{\varepsilon}\right)}+\|\overline{\mathcal{U}}\|_{L^{2}\left(\mathcal{S}_{\varepsilon}\right)}, \quad\left\|\frac{d \mathcal{U}}{d \mathbf{s}}\right\|_{L^{2}\left(\mathcal{S}_{\varepsilon}\right)} \leq\left\|\frac{d \mathcal{U}^{h}}{d \mathbf{s}}\right\|_{L^{2}\left(\mathcal{S}_{\varepsilon}\right)}+\left\|\frac{d \overline{\mathcal{U}}}{d \mathbf{s}}\right\|_{L^{2}\left(\mathcal{S}_{\varepsilon}\right)} .
$$

From the estimates of Lemmas 7-22 we obtain $(5.18)_{1,2}$. Estimate (5.12) yields

$$
\left\|\mathcal{R}^{h}\right\|_{L^{2}(\varepsilon(\xi+\mathcal{S}))}^{2} \leq \frac{C \varepsilon^{2}}{r^{4}}\|e(u)\|_{L^{2}\left(\varepsilon\left(\xi+\mathcal{S}_{r / \varepsilon}\right)\right)}^{2}+C|\mathbf{b}(\varepsilon \xi)|^{2} \varepsilon^{2} .
$$

Summing up over all $\xi \in \Xi_{\varepsilon}$ and applying (A.3) give

$$
\left\|\mathcal{R}^{h}\right\|_{L^{2}\left(\mathcal{S}_{\varepsilon}\right)} \leq C \frac{\varepsilon}{r^{2}}\|e(u)\|_{L^{2}\left(\mathcal{S}_{\varepsilon, r}\right)} .
$$


Then, this inequality together with the estimates (5.10) yield $(5.18)_{2}$.

From Definition 3 we have

$$
\begin{aligned}
& \left\|U^{e}\right\|_{L^{2}\left(\mathcal{S}_{\varepsilon, r}\right)} \leq C r\|\mathcal{U}\|_{L^{2}\left(\mathcal{S}_{\varepsilon}\right)}+C r^{2}\|\mathcal{R}\|_{L^{2}\left(\mathcal{S}_{\varepsilon}\right)}, \\
& \left\|\nabla U^{e}\right\|_{L^{2}\left(\mathcal{S}_{\varepsilon, r}\right)} \leq C\left(r\left\|\frac{d \mathcal{U}}{d \mathbf{s}}\right\|_{L^{2}\left(\mathcal{S}_{\varepsilon}\right)}+r^{2}\left\|\frac{d \mathcal{R}}{d \mathbf{s}}\right\|_{L^{2}\left(\mathcal{S}_{\varepsilon}\right)}+r\|\mathcal{R}\|_{L^{2}\left(\mathcal{S}_{\varepsilon}\right)}\right) .
\end{aligned}
$$

Then, the estimates $(5.18)_{1,2}$ and $(5.18)_{2}$ lead to $(5.18)_{3,4}$.

\section{Appendix B: The Applied Forces}

First, note that the number of elements in $\mathcal{K}_{\varepsilon}$, which is denoted by $\left|\mathcal{K}_{\varepsilon}\right|$ is less than

$$
\left|\mathcal{K}_{\varepsilon}\right| \leq \frac{|\mathcal{K}| \text { mes }\left|\Omega_{1}\right|}{\varepsilon^{3}}
$$

where $|\mathcal{K}|$ is the number of elements in $\mathcal{K}$.

Proof of Lemma 10 Let $u$ be in $\mathbf{V}_{\varepsilon, r}$. By the estimates of Proposition 2, we have

$$
\left|\sum_{\xi \in \Xi_{\varepsilon}} \int_{\mathcal{P}_{\varepsilon \ell, r}^{\xi}} \mathbf{f}_{\varepsilon} \cdot u d x\right| \leq C\|\mathbf{f}\|_{L^{\infty}(\Omega)}\|e(u)\|_{L^{2}\left(\mathcal{S}_{\varepsilon, r}\right)} .
$$

Now, taking into account that for every node $A \in \mathcal{K}_{\varepsilon}$ the following decomposition holds:

$$
u(x)=\mathcal{U}(A)+\mathcal{R}(A) \wedge(x-A)+\bar{u}, \quad \text { for a.e. } x \in B(A, r),
$$

we have

$$
\begin{aligned}
& \int_{\mathcal{S}_{\varepsilon, r}}\left(\sum_{A \in \mathcal{K}_{\varepsilon}} F(A) \mathbf{1}_{B(A, r)}+\sum_{A \in \mathcal{K}_{\varepsilon}} G(A) \wedge(x-A) \mathbf{1}_{B(A, r)}\right) \cdot u d x \\
= & \sum_{A \in \mathcal{K}_{\varepsilon}} \int_{B(A, r)} F(A) \cdot \mathcal{U}(A) d x+\sum_{A \in \mathcal{K}_{\varepsilon}} \int_{B(A, r)} F(A) \cdot(\mathcal{R}(A) \wedge(x-A)) d x \\
+ & \sum_{A \in \mathcal{K}_{\varepsilon}} \int_{B(A, r)}(G(A) \wedge(x-A)) \cdot \mathcal{U}(A) d x \\
+ & \sum_{A \in \mathcal{K}_{\varepsilon}} \int_{B(A, r)}(G(A) \wedge(x-A)) \cdot(\mathcal{R}(A) \wedge(x-A)) d x \\
+ & \sum_{A \in \mathcal{K}_{\varepsilon}} \int_{B(A, r)} F(A) \cdot \bar{u} d x+\sum_{A \in \mathcal{K}_{\varepsilon}} \int_{B(A, r)}(G(A) \wedge(x-A)) \cdot \bar{u} d x .
\end{aligned}
$$

Let us estimate every integral in (B.3) separately. Due to the symmetries of the ball we have

$$
\begin{aligned}
& \int_{B(A, r)} F(A) \cdot(\mathcal{R}(A) \wedge(x-A)) d x=0, \\
& \int_{B(A, r)}(G(A) \wedge(x-A)) \cdot \mathcal{U}(A) d x=0, \quad \forall A \in \mathcal{K} .
\end{aligned}
$$


Thus, the second and third terms in the right-hand side of (B.3) vanish. Then, using the Cauchy-Schwarz inequality, (5.9) ${ }_{1}$ and (B.1), the last two integrals in (B.3) are estimated as follows

$$
\begin{aligned}
& \left|\sum_{A \in \mathcal{K}_{\varepsilon}} \int_{B(A, r)} F(A) \cdot \bar{u} d x\right| \leq\left(\sum_{A \in \mathcal{K}_{\varepsilon}} \int_{B(A, r)}|F(A)|^{2} d x\right)^{1 / 2}\left(\int_{\mathcal{S}_{\varepsilon, r}}|\bar{u}|^{2} d x\right)^{1 / 2} \\
& \leq C\left(\frac{r}{\varepsilon}\right)^{3 / 2}\|F\|_{L^{\infty}(\Omega)}\|\bar{u}\|_{L^{2}\left(\mathcal{S}_{\varepsilon, r}\right)} \leq C r\left(\frac{r}{\varepsilon}\right)^{3 / 2}\|F\|_{L^{\infty}(\Omega)}\|e(u)\|_{L^{2}\left(\mathcal{S}_{\varepsilon, r}\right)}
\end{aligned}
$$

and

$$
\begin{aligned}
& \left|\sum_{A \in \mathcal{K}_{\varepsilon}} \int_{B(A, r)}(G(A) \wedge(x-A)) \cdot \bar{u} d x\right| \\
& \quad \leq\left(\sum_{A \in \mathcal{K}_{\varepsilon}} \int_{B(A, r)} r^{2}|G(A)|^{2} d x\right)^{1 / 2}\left(\int_{\mathcal{S}_{\varepsilon, r}}|\bar{u}|^{2} d x\right)^{1 / 2} \\
& \quad \leq C r\left(\frac{r}{\varepsilon}\right)^{3 / 2}\|G\|_{L^{\infty}(\Omega)}\|\bar{u}\|_{L^{2}\left(\mathcal{S}_{\varepsilon, r}\right)} \leq C r^{2}\left(\frac{r}{\varepsilon}\right)^{3 / 2}\|G\|_{L^{\infty}(\Omega)}\|e(u)\|_{L^{2}\left(\mathcal{S}_{\varepsilon, r}\right)}
\end{aligned}
$$

Since $\mathcal{U}^{h}(A)=\mathcal{U}(A)$ and $\mathcal{R}^{h}(A)=\mathcal{R}(A)$, then using the fact that $\mathcal{U}^{h}, \mathcal{R}^{h}$ are affine functions between two contiguous nodes

$$
\sum_{A \in \mathcal{K}_{\varepsilon}}\left|\mathcal{U}^{h}(A)\right|^{2} \varepsilon \leq C\left\|\mathcal{U}^{h}\right\|_{L^{2}\left(\mathcal{S}_{\varepsilon}\right)}^{2}, \quad \sum_{A \in \mathcal{K}_{\varepsilon}}\left|\mathcal{R}^{h}(A)\right|^{2} \varepsilon \leq C\left\|\mathcal{R}^{h}\right\|_{L^{2}\left(\mathcal{S}_{\varepsilon}\right)}^{2}
$$

Then, the remaining two integrals in the right-hand side of (B.3) are estimated using (B.1), $(\text { A.2 })_{6},(5.18)_{2}$ and (B.4)

$$
\begin{aligned}
& \left|\sum_{A \in \mathcal{K}_{\varepsilon}} \int_{B(A, r)} F(A) \cdot \mathcal{U}(A) d x\right| \\
& \quad \leq\left(\sum_{A \in \mathcal{K}_{\varepsilon}} \int_{B(A, r)}|F(A)|^{2} d x\right)^{1 / 2}\left(\sum_{A \in \mathcal{K}_{\varepsilon}} \int_{B(A, r)}|\mathcal{U}(A)|^{2} d x\right)^{1 / 2} \\
& \quad \leq C \frac{r^{3 / 2}}{\varepsilon^{3 / 2}}\|F\|_{L^{\infty}(\Omega)}\left(\frac{r^{3}}{\varepsilon} \sum_{A \in \mathcal{K}_{\varepsilon}}\left|\mathcal{U}^{h}(A)\right|^{2} \varepsilon\right)^{1 / 2} \leq C \frac{r^{3}}{\varepsilon^{2}}\|F\|_{L^{\infty}(\Omega)}\left\|\mathcal{U}^{h}\right\|_{L^{2}\left(\mathcal{S}_{\varepsilon}\right)} \\
& \quad \leq C \frac{r^{2}}{\varepsilon^{2}}\|F\|_{L^{\infty}(\Omega)}\|e(u)\|_{L^{2}\left(\mathcal{S}_{\varepsilon, r}\right)}
\end{aligned}
$$

and

$$
\begin{aligned}
& \left|\sum_{A \in \mathcal{K}_{\varepsilon}} \int_{B(A, r)}(G(A) \wedge(x-A)) \cdot(\mathcal{R}(A) \wedge(x-A)) d x\right| \\
& \leq\left(\sum_{A \in \mathcal{K}_{\varepsilon}} \int_{B(A, r)}|G(A) \wedge(x-A)|^{2} d x\right)^{1 / 2}\left(\sum_{A \in \mathcal{K}_{\varepsilon}} \int_{B(A, r)}|\mathcal{R}(A) \wedge(x-A)|^{2} d x\right)^{1 / 2} \\
& \leq\left(\sum_{A \in \mathcal{K}_{\varepsilon}} \int_{B(A, r)} r^{2}|G(A)|^{2} d x\right)^{1 / 2}\left(\sum_{A \in \mathcal{K}_{\varepsilon}} \int_{B(A, r)} r^{2}|\mathcal{R}(A)|^{2} d x\right)^{1 / 2}
\end{aligned}
$$




$$
\begin{aligned}
& \leq C \frac{r^{5 / 2}}{\varepsilon^{3 / 2}}\|G\|_{L^{\infty}(\Omega)}\left(\frac{r^{5}}{\varepsilon} \sum_{A \in \mathcal{K}_{\varepsilon}}\left|\mathcal{R}^{h}(A)\right|^{2} \varepsilon\right)^{1 / 2} \\
& \leq C \frac{r^{5}}{\varepsilon^{2}}\|G\|_{L^{\infty}(\Omega)}\left\|\mathcal{R}^{h}\right\|_{L^{2}\left(\mathcal{S}_{\varepsilon}\right)} \leq C \frac{r^{3}}{\varepsilon}\|G\|_{L^{\infty}(\Omega)}\|e(u)\|_{L^{2}\left(\mathcal{S}_{\varepsilon, r}\right)} .
\end{aligned}
$$

The above estimates, those of Lemma 22 and the fact that $r \leq \varepsilon$ end the proof of Lemma 10 .

\section{Appendix C: Unfolding Method Results}

Proof of Lemma 13 Since $\Phi_{\varepsilon}$ belongs to $W^{1, \infty}\left(\Omega_{\varepsilon}\right)$ then $\Phi_{\left.\varepsilon\right|_{\mathcal{S}_{\varepsilon}}}$ is in $W^{1, \infty}\left(\mathcal{S}_{\varepsilon}\right)$. Taking into account that $x=\varepsilon \xi+\varepsilon \mathbf{A}^{\ell}+s \mathbf{t}_{1}$ in $\mathcal{S}_{\varepsilon}$, we have equality $(6.10)_{2}$.

Since $Q_{1}(Y)$ is a finite dimensional vector space, there exist two strictly positive constants $c$ and $C$ such that for every $\Psi \in Q_{1}(Y)$

$$
\begin{aligned}
& c\left\|\Psi_{\mid \mathcal{S}}\right\|_{L^{2}(\mathcal{S})} \leq\|\Psi\|_{L^{2}(Y)} \leq C\left\|\Psi_{\mid \mathcal{S}}\right\|_{L^{2}(\mathcal{S})}, \\
& c\left\|\frac{d \Psi_{\mid \mathcal{S}}}{d \mathbf{S}}\right\|_{L^{2}(\mathcal{S})} \leq\|\nabla \Psi\|_{L^{2}(Y)} \leq C\left\|\frac{d \Psi_{\mid \mathcal{S}}}{d \mathbf{S}}\right\|_{L^{2}(\mathcal{S})} .
\end{aligned}
$$

Now, for every $\Phi \in W^{1, \infty}\left(\Omega_{\varepsilon}\right)$ satisfying (6.9), after $\varepsilon$-scaling, we obtain

$$
\begin{aligned}
c \varepsilon^{2}\left\|\Phi_{\mid \mathcal{S}_{\varepsilon}}\right\|_{L^{2}\left((\varepsilon \xi+\varepsilon \bar{Y}) \cap \mathcal{S}_{\varepsilon}\right)}^{2} & \leq\|\Phi\|_{L^{2}(\varepsilon \xi+\varepsilon Y)}^{2} \leq C \varepsilon^{2}\left\|\Phi_{\mid \mathcal{S}_{\varepsilon}}\right\|_{L^{2}\left((\varepsilon \xi+\varepsilon \bar{Y}) \cap \mathcal{S}_{\varepsilon}\right)}^{2}, \quad \\
c \varepsilon^{2}\left\|\frac{d \Phi_{\mid \mathcal{S}_{\varepsilon}}}{d \mathbf{s}}\right\|_{L^{2}\left((\varepsilon \xi+\varepsilon \bar{Y}) \cap \mathcal{S}_{\varepsilon}\right)}^{2} & \leq\|\nabla \Phi\|_{L^{2}(\varepsilon \xi+\varepsilon Y)}^{2} \leq C \varepsilon^{2}\left\|\frac{d \Phi_{\mid \mathcal{S}_{\varepsilon}}}{d \mathbf{s}}\right\|_{L^{2}\left((\varepsilon \xi+\varepsilon \bar{Y}) \cap \mathcal{S}_{\varepsilon}\right)}^{2}, \quad \forall \xi \in \Xi_{\varepsilon} .
\end{aligned}
$$

Summing up all these inequalities for all $\xi \in \boldsymbol{\Xi}_{\varepsilon}$ yields $(6.10)_{1,3}$.

Now, suppose that the sequence $\left\{\Phi_{\varepsilon}\right\}_{\varepsilon}$ of functions belonging to $W^{1, \infty}\left(\Omega_{\varepsilon}\right)$ satisfies (6.11). Then, up to a sequence of $\varepsilon$, there exists $\Phi \in L^{2}(\Omega)$ such that $(6.12)_{1}$ holds and furthermore due to (6.3) (see also [9, Theorem 3.6]), one has

$$
\mathcal{T}_{\varepsilon}^{\text {ext }}\left(\Phi_{\varepsilon}\right) \rightarrow \Phi \quad \text { weakly in } L^{2}(\Omega \times Y) .
$$

But, taking into account (6.9), we have the convergence $(6.12)_{2}$ which implies $(6.12)_{3}$, since the embedding $Q_{1}(Y) \subset H^{1}(\mathcal{S})$ is continuous.

Moreover, if $\left\|\Phi_{\varepsilon}\right\|_{H^{1}\left(\Omega_{\varepsilon}\right)} \leq C$ then, up to a sequence of $\varepsilon$, there exists $\Phi \in H^{1}(\Omega)$ such that $(6.13)_{1}$ holds. In the same way as [9, Theorem 3.6], we obtain convergence $(6.13)_{2}$, from which, taking into account $(6.10)_{1}$, we have convergence $(6.13)_{3}$.

Proof of Lemma 14 Using the properties of the unfolding operator $\mathcal{T}_{\varepsilon}^{\mathcal{S}}$ (6.3)-(6.4) and the estimates for $\phi_{\varepsilon}$, we obtain

$$
\left\|\mathcal{T}_{\varepsilon}^{\mathcal{S}}\left(\phi_{\varepsilon}\right)\right\|_{L^{2}\left(\Omega_{\varepsilon} \times \mathcal{S}\right)}=\varepsilon\left\|\phi_{\varepsilon}\right\|_{L^{2}\left(\mathcal{S}_{\varepsilon}\right)} \leq C,
$$

and

$$
\left\|\frac{\partial \mathcal{T}_{\varepsilon}^{\mathcal{S}}\left(\phi_{\varepsilon}\right)}{\partial \mathbf{S}}\right\|_{L^{2}\left(\Omega_{\varepsilon} \times \mathcal{S}\right)}=\left\|\varepsilon \mathcal{T}_{\mathcal{\varepsilon}}^{\mathcal{S}}\left(\frac{d \phi_{\varepsilon}}{d \mathbf{s}}\right)\right\|_{L^{2}\left(\Omega_{\varepsilon} \times \mathcal{S}\right)}=\varepsilon^{2}\left\|\frac{d \phi_{\varepsilon}}{d \mathbf{s}}\right\|_{L^{2}\left(\mathcal{S}_{\varepsilon}\right)} \leq C .
$$


Thus,

$$
\left\|\mathcal{T}_{\varepsilon}^{\mathcal{S}}\left(\phi_{\varepsilon}\right)\right\|_{L^{2}\left(\Omega ; H^{1}(\mathcal{S})\right)} \leq\left\|\mathcal{T}_{\varepsilon}^{\mathcal{S}}\left(\phi_{\varepsilon}\right)\right\|_{L^{2}\left(\Omega_{\varepsilon} ; H^{1}(\mathcal{S})\right)} \leq C .
$$

Hence, up to a subsequence $\varepsilon$, there exists $\widehat{\phi} \in L^{2}\left(\Omega ; H^{1}(\mathcal{S})\right)$ such that (6.14) holds.

In order to prove of (6.15), first observe that $\mathcal{T}_{\varepsilon}^{\mathcal{S}}\left(\phi_{\varepsilon} \mathbf{1}_{\widehat{\Omega}_{\varepsilon} \text { int }}\right)$ belongs to $L^{2}\left(\Omega ; H^{1}(\mathcal{S})\right)$ and

$$
\left\|\mathcal{T}_{\varepsilon}^{\mathcal{S}}\left(\phi_{\varepsilon}\right) \mathbf{1}_{\widehat{\Omega}_{\varepsilon}^{i n t} \times \mathcal{S}}\right\|_{L^{2}\left(\Omega ; H^{1}(\mathcal{S})\right)} \leq \varepsilon\left(\left\|\phi_{\varepsilon}\right\|_{L^{2}\left(\mathcal{S}_{\varepsilon} \cap \Omega_{\varepsilon}^{i n t}\right)}+\varepsilon\left\|\frac{d \phi_{\varepsilon}}{d \mathbf{s}}\right\|_{L^{2}\left(\mathcal{S}_{\varepsilon} \cap \Omega_{\varepsilon}^{i n t}\right)}\right) \leq C .
$$

And, up to a subsequence of $\{\varepsilon\}$, there exists $\widehat{\phi} \in L^{2}\left(\Omega ; H^{1}(\mathcal{S})\right.$ such that $(6.15)$ holds.

In both cases, the periodicity of $\widehat{\phi}$ is obtained proceeding in the same way as to prove [10, Theorem 4.28].

Proof of Lemma 15 The Poincaré-Wirtinger inequality gives a constant such that

$$
\forall \psi \in H^{1}(\mathcal{S}), \quad\left\|\psi-\frac{1}{|\mathcal{S}|} \int_{\mathcal{S}} \psi d \mathbf{S}\right\|_{L^{2}(\mathcal{S})} \leq C\left\|\frac{d \psi}{d \mathbf{S}}\right\|_{L^{2}(\mathcal{S})} .
$$

We apply the above inequality with the function $\psi(S)=\phi_{\varepsilon}(\varepsilon \xi+\varepsilon S), \xi \in \Xi_{\varepsilon}$. Then, after summation over $\xi \in \Xi_{\varepsilon}$, that yields

$$
\left\|\phi_{\varepsilon}-\mathcal{M}_{\varepsilon}^{*}\left(\phi_{\varepsilon}\right)\right\|_{L^{2}\left(\mathcal{S}_{\varepsilon}\right)} \leq C \varepsilon\left\|\frac{d \phi_{\varepsilon}}{d \mathbf{s}}\right\|_{L^{2}\left(\mathcal{S}_{\varepsilon}\right)} .
$$

Now, consider the function $\Phi_{\varepsilon}$ defined in the cell $\varepsilon(\xi+\bar{Y}), \xi \in \widehat{\Xi}_{\varepsilon}$, as the $Q_{1}$ interpolation of $\mathcal{M}_{\varepsilon}^{*}(\phi)(\varepsilon \xi)$ on the vertices of this parallelotope. One has

$$
\Phi_{\varepsilon} \in W^{1, \infty}\left(\widehat{\Omega}_{\varepsilon}\right) .
$$

Observe that $\Phi_{\varepsilon}$ also belongs to $W^{1, \infty}\left(\Omega_{\varepsilon}^{i n t}\right)$. Proceeding as in [10, Chap. 4] we obtain the following estimates:

$$
\begin{aligned}
& \left\|\Phi_{\varepsilon}\right\|_{L^{2}\left(\widehat{\Omega}_{\varepsilon}\right)} \leq C \varepsilon\left\|\phi_{\varepsilon}\right\|_{L^{2}\left(\mathcal{S}_{\varepsilon}\right)}, \quad\left\|\nabla \Phi_{\varepsilon}\right\|_{L^{2}\left(\widehat{\Omega}_{\varepsilon}\right)} \leq C \varepsilon\left\|\frac{d \phi_{\varepsilon}}{d \mathbf{s}}\right\|_{L^{2}\left(\mathcal{S}_{\varepsilon}\right)}, \\
& \left\|\Phi_{\varepsilon}-\mathcal{M}_{\varepsilon}^{*}\left(\phi_{\varepsilon}\right)\right\|_{L^{2}\left(\widehat{\Omega}_{\varepsilon}\right)} \leq C \varepsilon^{2}\left\|\frac{d \phi_{\varepsilon}}{d \mathbf{s}}\right\|_{L^{2}\left(\mathcal{S}_{\varepsilon}\right)} .
\end{aligned}
$$

Therefore,

$$
\left\|\Phi_{\varepsilon}\right\|_{H^{1}\left(\Omega_{\varepsilon}^{i n t}\right)} \leq\left\|\Phi_{\varepsilon}\right\|_{H^{1}\left(\widehat{\Omega}_{\varepsilon}\right)} \leq C .
$$

Lemma 5.1 in [16] gives $\Phi \in H^{1}(\Omega)$ such that (up to a subsequence)

$$
\begin{aligned}
& \Phi_{\varepsilon} \mathbf{1}_{\Omega_{\varepsilon}^{i n t}} \longrightarrow \Phi \quad \text { strongly in } \quad L^{2}(\Omega), \\
& \nabla \Phi_{\varepsilon} \mathbf{1}_{\Omega_{\varepsilon}^{i n t}} \rightarrow \nabla \Phi \quad \text { weakly in } \quad L^{2}(\Omega)^{3} .
\end{aligned}
$$

Besides, by definition of $\Phi_{\varepsilon}, \Phi_{\varepsilon \mid \widehat{\Omega}_{\varepsilon} \cap \mathcal{S}_{\varepsilon}}$ belongs to $W^{1, \infty}\left(\widehat{\Omega}_{\varepsilon} \cap \mathcal{S}_{\varepsilon}\right)$ and Lemma 13 gives

$$
\frac{d \Phi_{\varepsilon \mid \mathcal{S}_{\varepsilon}}}{d \mathbf{s}}=\nabla \Phi_{\varepsilon} \cdot \mathbf{t}_{1} \quad \text { a.e. in } \widehat{\Omega}_{\varepsilon} \cap \mathcal{S}_{\varepsilon}, \quad\left\|\frac{d \Phi_{\varepsilon \mid \mathcal{S}_{\varepsilon}}}{d \mathbf{s}}\right\|_{L^{2}\left(\widehat{\Omega}_{\varepsilon} \cap \mathcal{S}_{\varepsilon}\right)} \leq \frac{C}{\varepsilon}\left\|\nabla \Phi_{\varepsilon}\right\|_{L^{2}\left(\widehat{\Omega}_{\varepsilon}\right)} .
$$


Hence,

$$
\begin{aligned}
& \left\|\frac{d \Phi_{\varepsilon}}{d \mathbf{s}}-\frac{d \phi_{\varepsilon}}{d \mathbf{s}}\right\|_{L^{2}\left(\widehat{\Omega}_{\varepsilon} \cap \mathcal{S}_{\varepsilon}\right)} \\
& \quad \leq\left\|\frac{d \Phi_{\varepsilon}}{d \mathbf{s}}\right\|_{L^{2}\left(\widehat{\Omega}_{\varepsilon} \cap \mathcal{S}_{\varepsilon}\right)}+\left\|\frac{d \phi_{\varepsilon}}{d \mathbf{s}}\right\|_{L^{2}\left(\mathcal{S}_{\varepsilon}\right)} \\
& \quad \leq \frac{C}{\varepsilon}\left\|\nabla \Phi_{\varepsilon}\right\|_{L^{2}\left(\widehat{\Omega}_{\varepsilon}\right)}+\left\|\frac{d \phi_{\varepsilon}}{d \mathbf{s}}\right\|_{L^{2}\left(\mathcal{S}_{\varepsilon}\right)} \leq C\left\|\frac{d \phi_{\varepsilon}}{d \mathbf{s}}\right\|_{L^{2}\left(\mathcal{S}_{\varepsilon}\right)} \leq \frac{C}{\varepsilon} .
\end{aligned}
$$

By (6.16), (C.1)-(C.2), we obtain

$$
\left\|\Phi_{\varepsilon}-\phi_{\varepsilon}\right\|_{L^{2}\left(\widehat{\Omega}_{\varepsilon} \cap \mathcal{S}_{\varepsilon}\right)} \leq C \varepsilon\left\|\frac{d \phi_{\varepsilon}}{d \mathbf{s}}\right\|_{L^{2}\left(\mathcal{S}_{\varepsilon}\right)} \leq C
$$

Therefore, Lemma 14 gives a function $\widehat{\phi} \in L^{2}\left(\Omega ; H_{p e r}^{1}(\mathcal{S})\right)$ such that (up to a subsequence)

$$
\frac{1}{\varepsilon} \mathcal{T}_{\varepsilon}^{\mathcal{S}}\left(\phi_{\varepsilon}-\Phi_{\varepsilon}\right) \mathbf{1}_{\widehat{\Omega}_{\varepsilon}^{i n t} \times \mathcal{S}} \rightarrow \widehat{\phi} \quad \text { weakly in } L^{2}\left(\Omega ; H^{1}(\mathcal{S})\right)
$$

Due to estimate (C.4), there exist a subsequence of $\{\varepsilon\}$ and $F \in L^{2}(\mathcal{S})$ such that

$$
\mathcal{T}_{\varepsilon}^{\mathcal{S}}\left(\frac{d \Phi_{\varepsilon}}{d \mathbf{s}}\right) \mathbf{1}_{\widehat{\Omega}_{\varepsilon} \times \mathcal{S}} \rightarrow F \quad \text { weakly in } L^{2}(\Omega \times \mathcal{S})
$$

Let $\mathcal{O}$ be an open set strictly included in $\Omega$. If $\varepsilon$ is small enough, one has

$$
\mathcal{O} \subset \widehat{\Omega}_{\varepsilon}^{i n t} \subset \widehat{\Omega}_{\varepsilon}
$$

Applying Lemma 13 in the context of the open set $\mathcal{O}$ leads to (up to a subsequence)

$$
\mathcal{T}_{\varepsilon}^{\mathcal{S}}\left(\frac{d \Phi_{\varepsilon}}{d \mathbf{s}}\right) \mathbf{1}_{\mathcal{O} \times \mathcal{S}}=\mathcal{T}_{\varepsilon}^{\text {ext }}\left(\nabla \Phi_{\varepsilon} \cdot \mathbf{t}_{1}\right) \mathbf{1}_{\mathcal{O} \times \mathcal{S}} \rightarrow \nabla \Phi \cdot \mathbf{t}_{1} \quad \text { weakly in } L^{2}(\mathcal{O} \times \mathcal{S}) .
$$

Hence,

$$
F=\nabla \Phi \cdot \mathbf{t}_{1} \quad \text { a.e. in } \mathcal{O} \times \mathcal{S} .
$$

As a consequence $F=\nabla \Phi \cdot \mathbf{t}_{1}$ a.e. in $\Omega \times \mathcal{S}$ and (6.17) are proved. Now, from (C.3) and (C.5) we obtain

$$
\begin{aligned}
& \mathcal{T}_{\varepsilon}^{\mathcal{S}}\left(\Phi_{\varepsilon}\right) \mathbf{1}_{\widehat{\Omega}_{\varepsilon}^{i n t} \times \mathcal{S}} \longrightarrow \Phi \quad \text { strongly in } \quad L^{2}\left(\Omega ; H^{1}(\mathcal{S})\right) \\
& \mathcal{T}_{\varepsilon}^{\mathcal{S}}\left(\phi_{\varepsilon}-\Phi_{\varepsilon}\right) \mathbf{1}_{\widehat{\Omega}_{\varepsilon}{ }_{i n} \times \mathcal{S}} \longrightarrow 0 \quad \text { strongly in } \quad L^{2}\left(\Omega ; H^{1}(\mathcal{S})\right)
\end{aligned}
$$

Hence,

$$
\mathcal{T}_{\varepsilon}^{\mathcal{S}}\left(\phi_{\varepsilon}\right) \mathbf{1}_{\widehat{\Omega}_{\varepsilon}^{i n t} \times \mathcal{S}} \longrightarrow \Phi \quad \text { strongly in } \quad L^{2}\left(\Omega ; H^{1}(\mathcal{S})\right)
$$




\section{Appendix D: Proof of Lemma 16}

Step 1. We show that the semi-norm $\|\cdot\|_{\mathcal{S}}$ is a norm in $\mathcal{D}_{E x}$.

Indeed, if $\left\|\frac{d \overline{\mathcal{A}}}{d \mathbf{S}} \cdot \mathbf{t}_{1}\right\|_{L^{2}(\mathcal{S})}=0$ then $\overline{\mathcal{A}}$ is a rigid displacement (remind that $\mathcal{S}$ is a stable structure). The periodicity of $\overline{\mathcal{A}}$ implies that $\overline{\mathcal{A}}$ is a constant field. Since the mean value of $\overline{\mathcal{A}}$ is equal to zero then $\overline{\mathcal{A}}=0$. Hence, the semi-norm $\|\cdot\|_{\mathcal{S}}$ is a norm in $\mathcal{D}_{E x}$.

Step 2. We show that the norm $\|\cdot\|_{\mathcal{S}}$ is equivalent to the norm $\|\cdot\|_{H^{1}(\mathcal{S})}$.

First, we have

$$
\forall \overline{\mathcal{A}} \in \mathcal{D}_{E x}, \quad\|\overline{\mathcal{A}}\|_{\mathcal{S}}=\left\|\frac{d \overline{\mathcal{A}}}{d \mathbf{S}} \cdot \mathbf{t}_{1}\right\|_{L^{2}(\mathcal{S})} \leq\left\|\frac{d \overline{\mathcal{A}}}{d \mathbf{S}}\right\|_{L^{2}(\mathcal{S})} \leq\|\overline{\mathcal{A}}\|_{H^{1}(\mathcal{S})} .
$$

The map

$$
\overline{\mathcal{A}} \in \mathcal{D}_{E x} \longmapsto \overline{\mathcal{A}}_{\mathrm{Aff}} \in H_{\text {per }}^{1}(\mathcal{S})^{3} \cap \mathbf{U}_{\mathcal{S}}
$$

where $\overline{\mathcal{A}}_{\text {Aff }}$ is defined by

$$
\overline{\mathcal{A}}_{\mathrm{Aff}}(A)=\overline{\mathcal{A}}(A) \quad \forall A \in \mathcal{K} .
$$

Lemma 1 claims that there exists a rigid displacement $\mathbf{r}$ such that

$$
\left\|\overline{\mathcal{A}}_{\mathrm{Aff}}-\mathbf{r}\right\|_{H^{1}(\mathcal{S})} \leq C\left\|\overline{\mathcal{A}}_{\mathrm{Aff}}\right\|_{\mathcal{S}}=C\left\|\frac{d \overline{\mathcal{A}}_{\mathrm{Aff}}}{d \mathbf{S}} \cdot \mathbf{t}_{1}\right\|_{L^{2}(\mathcal{S})} .
$$

Since $\mathcal{S}$ is a 3-periodic structure and $\overline{\mathcal{A}}_{\text {Aff }}$ is a periodic function, we can choose $\mathbf{r}$ constant. Hence,

$$
\left\|\frac{d \overline{\mathcal{A}}_{\mathrm{Aff}}}{d \mathbf{S}}\right\|_{L^{2}(\mathcal{S})} \leq C\left\|\frac{d \overline{\mathcal{A}}_{\mathrm{Aff}}}{d \mathbf{S}} \cdot \mathbf{t}_{1}\right\|_{L^{2}(\mathcal{S})} .
$$

The function $\overline{\mathcal{A}}-\overline{\mathcal{A}}_{\text {Aff }}$ vanishes on all the nodes. Therefore by the definitions of the functions $\overline{\mathcal{A}}$ and $\overline{\mathcal{A}}_{\text {Aff }}$ we obtain

$$
\left(\overline{\mathcal{A}}-\overline{\mathcal{A}}_{\mathrm{Aff}}\right) \wedge \mathbf{t}_{1} \text { is an affine function on all the segments } \gamma_{\ell}, \ell \in\{1, \ldots, m\}
$$

Hence,

$$
\left\|\frac{d\left(\overline{\mathcal{A}}-\overline{\mathcal{A}}_{\mathrm{Aff}}\right)}{d \mathbf{S}} \cdot \mathbf{t}_{1}\right\|_{L^{2}(\mathcal{S})}^{2}+\left\|\frac{d \overline{\mathcal{A}}_{\mathrm{Aff}}}{d \mathbf{S}} \cdot \mathbf{t}_{1}\right\|_{L^{2}(\mathcal{S})}^{2}=\left\|\frac{d \overline{\mathcal{A}}}{d \mathbf{S}} \cdot \mathbf{t}_{1}\right\|_{L^{2}(\mathcal{S})}^{2}
$$

and, therefore,

$$
\left\|\frac{d\left(\overline{\mathcal{A}}-\overline{\mathcal{A}}_{\mathrm{Aff}}\right)}{d \mathbf{S}}\right\|_{L^{2}(\mathcal{S})}=\left\|\frac{d\left(\overline{\mathcal{A}}-\overline{\mathcal{A}}_{\mathrm{Aff}}\right)}{d \mathbf{S}} \cdot \mathbf{t}_{1}\right\|_{L^{2}(\mathcal{S})} \leq\left\|\frac{d \overline{\mathcal{A}}}{d \mathbf{S}} \cdot \mathbf{t}_{1}\right\|_{L^{2}(\mathcal{S})} .
$$

As a consequence of (D.1)-(D.3), one obtains

$$
\forall \overline{\mathcal{A}} \in \mathcal{D}_{E x}, \quad\left\|\frac{d \overline{\mathcal{A}}}{d \mathbf{S}}\right\|_{L^{2}(\mathcal{S})} \leq C\left\|\frac{d \overline{\mathcal{A}}}{d \mathbf{S}} \cdot \mathbf{t}_{1}\right\|_{L^{2}(\mathcal{S})} .
$$


Remind that since $\overline{\mathcal{A}}$ belongs to $H_{p e r, 0}^{1}(\mathcal{S})^{3}$, the Poincaré-Wirtinger inequality gives

$$
\forall \overline{\mathcal{A}} \in \mathcal{D}_{E x}, \quad\|\overline{\mathcal{A}}\|_{L^{2}(\mathcal{S})} \leq C\left\|\frac{d \overline{\mathcal{A}}}{d \mathbf{S}}\right\|_{L^{2}(\mathcal{S})} \leq C\left\|\frac{d \overline{\mathcal{A}}}{d \mathbf{S}} \cdot \mathbf{t}_{1}\right\|_{L^{2}(\mathcal{S})} .
$$

Thus,

$$
\forall \overline{\mathcal{A}} \in \mathcal{D}_{E x}, \quad\|\overline{\mathcal{A}}\|_{H^{1}(\mathcal{S})} \leq C\left\|\frac{d \overline{\mathcal{A}}}{d \mathbf{S}} \cdot \mathbf{t}_{1}\right\|_{L^{2}(\mathcal{S})}=C\|\overline{\mathcal{A}}\|_{\mathcal{S}} .
$$

Both norms are equivalent.

Step 3. We show that the semi-norm $\|(\cdot, \cdot)\|_{\mathcal{D}_{I n}}$ is a norm in $\mathcal{D}_{I n}$.

Indeed, if $\left\|\frac{d \widehat{\mathcal{B}}}{d \mathbf{S}}\right\|_{L^{2}(\mathcal{S})}=0$, then $\widehat{\mathcal{B}}$ is a constant field. Remind that $\widehat{\mathcal{A}}$ vanishes on all the nodes, therefore one has $\widehat{\mathcal{B}} \wedge \mathbf{t}_{1}=0$ in $\mathcal{S}$. Since every node is a common extremity of at least two segments with non-collinear direction, then $\widehat{\mathcal{B}}$ vanishes on every node and thus $\widehat{\mathcal{B}}=0$ in $\mathcal{S}$. Hence, we have $\widehat{\mathcal{A}}=0$ on $\mathcal{S}$ and the semi-norm $\|(\cdot, \cdot)\|_{\mathcal{D}_{I n}}$ is a norm in $\mathcal{D}_{I n}$.

Step 4 . We show that the norm $\|(\cdot, \cdot)\|_{\mathcal{D}_{I n}}$ is equivalent to the norm $\|(\cdot, \cdot)\|_{H^{1}(\mathcal{S}) \times H^{1}(\mathcal{S})}$.

First, we have

$$
\begin{aligned}
& \forall(\widehat{\mathcal{A}}, \widehat{\mathcal{B}}) \in \mathcal{D}_{I n}, \\
& \|(\widehat{\mathcal{A}}, \widehat{\mathcal{B}})\|_{\mathcal{D}_{I n}} \doteq\left\|\frac{d \widehat{\mathcal{B}}}{d \mathbf{S}}\right\|_{L^{2}(\mathcal{S})} \leq\|\widehat{\mathcal{A}}\|_{H^{1}(\mathcal{S})}+\|\widehat{\mathcal{B}}\|_{H^{1}(\mathcal{S})}=\|(\widehat{\mathcal{A}}, \widehat{\mathcal{B}})\|_{H^{1}(\mathcal{S}) \times H^{1}(\mathcal{S})} .
\end{aligned}
$$

We prove by contradiction that there exists a constant $C$ strictly positive such that

$$
\forall(\widehat{\mathcal{A}}, \widehat{\mathcal{B}}) \in \mathcal{D}_{\text {In }}, \quad\|(\widehat{\mathcal{A}}, \widehat{\mathcal{B}})\|_{H^{1}(\mathcal{S}) \times H^{1}(\mathcal{S})} \leq C\|(\widehat{\mathcal{A}}, \widehat{\mathcal{B}})\|_{\mathcal{D}_{\text {In }}} .
$$

Suppose that such constant does not exist, then for every $n \geq 1$, there exists $\left(\widehat{\mathcal{A}}_{n}, \widehat{\mathcal{B}}_{n}\right) \in \mathcal{D}_{I n}$ such that

$$
\left\|\left(\widehat{\mathcal{A}}_{n}, \widehat{\mathcal{B}}_{n}\right)\right\|_{H^{1}(\mathcal{S}) \times H^{1}(\mathcal{S})}=1 \quad \text { and } \quad\left\|\left(\widehat{\mathcal{A}}_{n}, \widehat{\mathcal{B}}_{n}\right)\right\|_{\mathcal{D}_{I n}} \leq \frac{1}{n} .
$$

Thus, there exists a subsequence, still denoted $n$, such that

$$
\left(\widehat{\mathcal{A}}_{n}, \widehat{\mathcal{B}}_{n}\right) \rightarrow(\widehat{\mathcal{A}}, \widehat{\mathcal{B}}) \quad \text { weakly in }\left(H_{p e r}^{1}(\mathcal{S})^{3} \times H_{p e r}^{1}(\mathcal{S})^{3}\right) \cap \mathcal{D}_{\text {In }} .
$$

Then, one has

$$
\|(\widehat{\mathcal{A}}, \widehat{\mathcal{B}})\|_{\mathcal{D}_{\text {In }}}=\left\|\frac{d \widehat{\mathcal{B}}}{d \mathbf{S}}\right\|_{L^{2}(\mathcal{S})} \leq \liminf _{n \rightarrow+\infty}\left\|\frac{d \widehat{\mathcal{B}}_{n}}{d \mathbf{S}}\right\|_{L^{2}(\mathcal{S})}=\liminf _{n \rightarrow+\infty}\left\|\left(\widehat{\mathcal{A}}_{n}, \widehat{\mathcal{B}}_{n}\right)\right\|_{\mathcal{D}_{I n}}=0 .
$$

Hence, $\|(\widehat{\mathcal{A}}, \widehat{\mathcal{B}})\|_{\mathcal{D}_{I n}}=0$ which implies $(\widehat{\mathcal{A}}, \widehat{\mathcal{B}})=(0,0)$. As a consequence of the above convergences, the Sobolev embedding and the definition of $\mathcal{D}_{I n}$ we obtain

$$
\begin{array}{ll} 
& \widehat{\mathcal{B}}_{n} \longrightarrow 0 \quad \text { strongly in } H_{p e r}^{1}(\mathcal{S})^{3}, \\
\text { and then } \quad \widehat{\mathcal{A}}_{n} \longrightarrow 0 \quad \text { strongly in } H_{p e r}^{1}(\mathcal{S})^{3} .
\end{array}
$$

Finally $\left\|\left(\widehat{\mathcal{A}}_{n}, \widehat{\mathcal{B}}_{n}\right)\right\|_{H^{1}(\mathcal{S}) \times H^{1}(\mathcal{S})} \rightarrow 0$ which gives us a contradiction. 


\section{Appendix E: A Density Result for $H_{p e r}^{1}(\mathcal{S})$ and $\mathcal{D}_{I n}$}

Let $\mathfrak{r}$ and $a$ be two constants such that $0<4 \mathfrak{r}<a$.

Lemma 23 For every $\phi$ in $H^{1}(0, a)$, we define $\mathcal{F}_{\mathfrak{r}, a}(\phi) \in H^{1}(0, a)$ by

$$
\mathcal{F}_{\mathfrak{r}, a}(\phi)(t)=\left\{\begin{array}{l}
\phi(0) \\
\quad \text { if } t \in[0, \mathfrak{r}], \\
(\phi(2 \mathfrak{r})-\phi(0)) \frac{t-\mathfrak{r}}{\mathfrak{r}}+\phi(0)+A(t-\mathfrak{r})(t-2 \mathfrak{r}) \\
\quad \text { if } t \in[\mathfrak{r}, 2 \mathfrak{r}], \\
\phi(t) \\
\quad \text { if } t \in[2 \mathfrak{r}, a-2 \mathfrak{r}], \\
(\phi(a)-\phi(a-2 \mathfrak{r})) \frac{t-a+2 \mathfrak{r}}{\mathfrak{r}}+\phi(a)+B(t-a+\mathfrak{r})(t-a+2 \mathfrak{r}) \\
\quad \text { ift } \in[a-2 \mathfrak{r}, a-\mathfrak{r}], \\
\phi(a) \\
\quad \text { ift } \in[a-\mathfrak{r}, a],
\end{array}\right.
$$

where $A$ and $B$ are determined by the equalities

$$
\int_{0}^{2 \mathfrak{r}} \mathcal{F}_{\mathfrak{r}, a}(\phi)(t) d t=\int_{0}^{2 \mathfrak{r}} \phi(t) d t, \quad \int_{a-2 \mathfrak{r}}^{a} \mathcal{F}_{\mathfrak{r}, a}(\phi)(t) d t=\int_{a-2 \mathfrak{r}}^{a} \phi(t) d t
$$

Then one has

$$
\left\|\mathcal{F}_{\mathfrak{r}, a}(\phi)^{\prime}\right\|_{L^{2}(0,2 \mathfrak{r})}+\left\|\mathcal{F}_{\mathfrak{r}, a}(\phi)^{\prime}\right\|_{L^{2}(a-2 \mathfrak{r}, a)} \leq C\left(\left\|\phi^{\prime}\right\|_{L^{2}(0,2 \mathfrak{r})}+\left\|\phi^{\prime}\right\|_{L^{2}(a-2 \mathfrak{r}, a)}\right) .
$$

The constant does not depend on a and $\mathfrak{r}$.

Proof From (E.1) 1 we have

$$
\begin{aligned}
A & =\frac{6}{\mathfrak{r}^{3}}\left(\frac{\mathfrak{r}}{2}(\phi(2 \mathfrak{r})-\phi(0))+\int_{0}^{2 \mathfrak{r}}(\phi(0)-\phi(t)) d t\right) \\
& =\frac{6}{\mathfrak{r}^{3}}\left(\frac{\mathfrak{r}}{2} \int_{0}^{2 \mathfrak{r}} \phi^{\prime}(\tilde{t}) d \tilde{t}-\int_{0}^{2 \mathfrak{r}} \int_{0}^{t} \phi^{\prime}(\tilde{t}) d \tilde{t} d t\right) .
\end{aligned}
$$

Then, using the Cauchy-Schwartz inequality we obtain

$$
\begin{aligned}
|A|^{2} & \leq \frac{36}{\mathfrak{r}^{4}}\left(\mathfrak{r}\left\|\phi^{\prime}\right\|_{L^{2}(0,2 r)}^{2}+\int_{0}^{2 \mathfrak{r}} \int_{0}^{t}\left|\phi^{\prime}(\tilde{t})\right|^{2} d \tilde{t} d t\right) \leq \frac{36}{\mathfrak{r}^{4}}\left(\mathfrak{r}\left\|\phi^{\prime}\right\|_{L^{2}(0,2 r)}^{2}+2 \mathfrak{r}\left\|\phi^{\prime}\right\|_{L^{2}(0,2 r)}^{2}\right) \\
& =\frac{108}{\mathfrak{r}^{3}}\left\|\phi^{\prime}\right\|_{L^{2}(0,2 r)}^{2} .
\end{aligned}
$$


By definition of $\mathcal{F}_{\mathfrak{r}, a}$ and again using the Cauchy-Schwartz inequality we have

$$
\begin{aligned}
& \left\|\mathcal{F}_{\mathfrak{r}, a}(\phi)^{\prime}\right\|_{L^{2}(0,2 \mathfrak{r})}^{2}=\int_{\mathfrak{r}}^{2 \mathfrak{r}}\left(\frac{\phi(2 \mathfrak{r})-\phi(0)}{\mathfrak{r}}+A(t-\mathfrak{r})+A(t-2 \mathfrak{r})\right)^{2} d t \\
& \leq \frac{3}{\mathfrak{r}^{2}} \int_{\mathfrak{r}}^{2 \mathfrak{r}}(\phi(2 \mathfrak{r})-\phi(0))^{2} d t+3 A^{2} \int_{\mathfrak{r}}^{2 \mathfrak{r}}(t-\mathfrak{r})^{2} d t+3 A^{2} \int_{\mathfrak{r}}^{2 \mathfrak{r}}(t-2 \mathfrak{r})^{2} d t \\
& =\frac{3}{\mathfrak{r}}\left(\int_{0}^{2 \mathfrak{r}} \phi^{\prime}(\tilde{t}) d \tilde{t}\right)^{2} d t+2 A^{2} \mathfrak{r}^{3} \leq \frac{3}{\mathfrak{r}}\left(\int_{0}^{2 \mathfrak{r}} 1^{2} d t\right)\left(\int_{0}^{2 \mathfrak{r}}\left|\phi^{\prime}(\tilde{t})\right|^{2} d \tilde{t}\right)+204\left\|\phi^{\prime}\right\|_{L^{2}(0,2 r)}^{2} \\
& \leq 6\left\|\phi^{\prime}\right\|_{L^{2}(0,2 \mathfrak{r})}^{2}+204\left\|\phi^{\prime}\right\|_{L^{2}(0,2 r)}^{2}=210\left\|\phi^{\prime}\right\|_{L^{2}(0,2 \mathfrak{r})}^{2} .
\end{aligned}
$$

In the same way we obtain

$$
\left\|\mathcal{F}_{\mathfrak{r}, a}(\phi)^{\prime}\right\|_{L^{2}(a-2 \mathfrak{r}, a)}^{2} \leq 210\left\|\phi^{\prime}\right\|_{L^{2}(a-2 \mathfrak{r}, a)}^{2}
$$

and (E.2) holds.

Let $\mathcal{S}$ be a $3 D$-periodic structure. For every $\mathfrak{r}$ satisfying (remind that $l_{\ell}$ is the length of the segment $\gamma_{\ell} \subset \mathcal{S}$ )

$$
0<4 \mathfrak{r}<\min _{\ell \in\{1, \ldots, m\}} l_{\ell}
$$

we define the map $\mathcal{F}_{\mathfrak{r}}$ from $H^{1}(\mathcal{S})$ into $H^{1}(\mathcal{S})$ by

$$
\forall \phi \in H^{1}(\mathcal{S}), \quad \forall \gamma_{\ell} \subset \mathcal{S}, \quad \mathcal{F}_{\mathfrak{r}}(\phi)_{\mid \gamma_{\ell}}=\mathcal{F}_{\mathfrak{r}, l_{\ell}}\left(\phi_{\mid \gamma_{\ell}}\right) .
$$

Lemma $24 \mathcal{F}_{\mathfrak{r}}$ is a linear and continuous map from $H_{\text {per }}^{1}(\mathcal{S})$ into $H_{\text {per }}^{1}(\mathcal{S})$. We have

$$
\forall \phi \in H_{\text {per }}^{1}(\mathcal{S}), \quad \mathcal{F}_{\mathfrak{r}}(\phi) \longrightarrow \phi \quad \text { strongly in } H_{\text {per }}^{1}(\mathcal{S}) .
$$

For every $(\widehat{\mathcal{A}}, \widehat{\mathcal{B}}) \in \mathcal{D}_{\text {In }}$, we define $\widehat{\mathcal{A}}_{\mathfrak{r}} \in H_{\text {per }}^{1}(\mathcal{S})^{3}$ by

$$
\frac{d \widehat{\mathcal{A}}_{\mathfrak{r}}}{d \mathbf{s}}=\mathcal{F}_{\mathfrak{r}}(\widehat{\mathcal{B}}) \wedge \mathbf{t}_{1}, \quad \widehat{\mathcal{A}}_{\mathfrak{r}}=0 \text { on all the nodes of } \mathcal{S} .
$$

The couple $\left(\widehat{\mathcal{A}}_{\mathfrak{r}}, \mathcal{F}_{\mathfrak{r}}(\widehat{\mathcal{B}})\right)$ belongs to $\mathcal{D}_{\text {In }}$ and we have

$$
\begin{aligned}
\mathcal{F}_{\mathfrak{r}}(\widehat{\mathcal{B}}) & \longrightarrow \widehat{\mathcal{B}} \quad \text { strongly in } H_{\text {per }}^{1}(\mathcal{S})^{3}, \\
\widehat{\mathcal{A}_{\mathfrak{r}}} & \longrightarrow \widehat{\mathcal{A}} \quad \text { strongly in } H_{\text {per }}^{1}(\mathcal{S})^{3} .
\end{aligned}
$$

Proof First observe that for every $\phi \in H^{1}(\mathcal{S})$, the function $\mathcal{F}_{\mathfrak{r}}(\phi)-\phi$ vanishes on every nodes. As a consequence, $\mathcal{F}_{\mathfrak{r}}$ maps $H_{p e r}^{1}(\mathcal{S})$ into $H_{\text {per }}^{1}(\mathcal{S})$. Then (E.3) follows from Lemma 23.

Due to properties $\left(\right.$ E.1) of $\mathcal{F}_{\mathfrak{r}}(\widehat{\mathcal{B}})$, the function $\widehat{\mathcal{A}}_{\mathfrak{r}}$ is well define by (E.4). Then, the convergences (E.5) are the immediate consequences of Lemma 23. 


\section{Appendix F: The Test Functions $\phi_{\varepsilon, r}$ and $\mathcal{V}_{\varepsilon, r}$}

Let $\phi$ be in $\mathcal{D}(\Omega)$. We define the field $\phi_{\varepsilon, r}$ belonging to $W^{1, \infty}\left(\mathcal{S}_{\varepsilon}\right)$ as follows:for every $\xi \in \boldsymbol{\Xi}_{\varepsilon}$ and every $\gamma_{\ell}=\left[\mathbf{A}^{\ell}, \mathbf{A}^{\ell}+l_{\ell} \mathbf{t}_{1}^{\ell}\right] \in \mathcal{S}$ we set

$$
\phi_{\varepsilon, r}(\mathbf{s})= \begin{cases}\phi\left(\varepsilon \xi+\varepsilon \mathbf{A}^{\ell}\right) & \text { in }\left[-c_{0} r, c_{0} r\right], \\ \boldsymbol{\Phi}_{\varepsilon, r}\left(s_{1}\right) & \text { if } s_{1} \in\left[c_{0} r, \varepsilon l_{\ell}-c_{0} r\right], \\ \phi\left(\varepsilon \xi+\varepsilon \mathbf{B}^{\ell}\right) & \text { in }\left[\varepsilon l_{\ell}-c_{0} r, \varepsilon l_{\ell}+c_{0} r\right],\end{cases}
$$

where $\boldsymbol{\Phi}_{\varepsilon, r}$ is a polynomial function of degree less than 3 with respect to $s_{1}$ such that

$$
\begin{aligned}
& \boldsymbol{\Phi}_{\varepsilon, r}\left(c_{0} r\right)=\phi\left(\varepsilon \xi+\varepsilon \mathbf{A}^{\ell}\right), \quad \boldsymbol{\Phi}_{\varepsilon, r}\left(\varepsilon l_{\ell}-c_{0} r\right)=\phi\left(\varepsilon \xi+\varepsilon \mathbf{B}^{\ell}\right), \\
& \frac{d \boldsymbol{\Phi}_{\varepsilon, r}}{d s_{1}}\left(c_{0} r\right)=\frac{d \boldsymbol{\Phi}_{\varepsilon, r}}{d s_{1}}\left(\varepsilon l_{\ell}-c_{0} r\right)=0 .
\end{aligned}
$$

By construction $\phi_{\varepsilon,\left.r\right|_{\varepsilon \xi+\varepsilon \gamma_{\ell}}}$ belongs to $W^{2, \infty}\left(\varepsilon \xi+\varepsilon \gamma_{\ell}\right)$. We easily check that

$$
\begin{aligned}
& \mathcal{T}_{\varepsilon}^{\mathcal{S}}\left(\phi_{\varepsilon, r}\right) \longrightarrow \phi \quad \text { strongly in } \quad L^{2}\left(\Omega^{\prime} \times \mathcal{S}^{\prime}\right), \\
& \varepsilon \mathcal{T}_{\varepsilon}^{\mathcal{S}}\left(\frac{d \phi_{\varepsilon, r}}{d \mathbf{s}}\right), \quad \varepsilon^{2} \mathcal{T}_{\varepsilon}^{b, \ell}\left(\frac{d^{2} \phi_{\varepsilon, r}}{d \mathbf{s}^{2}}\right) \longrightarrow 0 \quad \text { strongly in } \quad L^{2}\left(\Omega^{\prime} \times \mathcal{S}^{\prime}\right) .
\end{aligned}
$$

Let $\mathcal{V}$ be in $\mathcal{D}\left(\mathbb{R}^{3}\right)$ such that $\mathcal{V}=0$ in $\Omega^{\prime} \backslash \bar{\Omega}$. We define the field $\phi_{\varepsilon, r}$ belonging to $W^{1, \infty}\left(\mathcal{S}_{\varepsilon}^{\prime}\right)$ as follows:for every $\xi \in \Xi_{\varepsilon}$ and every $\gamma_{\ell}=\left[\mathbf{A}^{\ell}, \mathbf{A}^{\ell}+l_{\ell} \mathbf{t}_{1}^{\ell}\right] \in \mathcal{S}$ we set

$$
\mathcal{V}_{\varepsilon, r}(\mathbf{s})=\left\{\begin{array}{l}
\mathcal{V}\left(\varepsilon \xi+\varepsilon \mathbf{A}^{\ell}\right) \\
\quad \text { in }\left[-c_{0} r, c_{0} r\right] \\
\mathcal{V}\left(\varepsilon \xi+\varepsilon \mathbf{A}^{\ell}\right)+\left(\mathcal{V}\left(\varepsilon \xi+\varepsilon \mathbf{A}^{\ell}+\varepsilon \mathbf{t}_{1}^{\ell}\right)-\mathcal{V}\left(\varepsilon \xi+\varepsilon \mathbf{A}^{\ell}\right)\right) \frac{s_{1}-c_{0} r}{\varepsilon l_{\ell}-2 c_{0} r} \\
\quad \text { if } s_{1} \in\left[c_{0} r, \varepsilon l_{\ell}-c_{0} r\right] \\
\mathcal{V}\left(\varepsilon \xi+\varepsilon \mathbf{B}^{\ell}\right) \\
\quad \text { in }\left[\varepsilon l_{\ell}-c_{0} r, \varepsilon l_{\ell}+c_{0} r\right] .
\end{array}\right.
$$

We easily check that

$$
\mathcal{T}_{\varepsilon}^{\mathcal{S}}\left(\frac{d \mathcal{V}_{\varepsilon, r}}{d \mathbf{s}}\right) \longrightarrow \nabla \mathcal{V} \cdot \mathbf{t}_{1} \quad \text { strongly in } \quad L^{2}\left(\Omega^{\prime} \times \mathcal{S}^{\prime}\right)
$$

\section{Appendix G: Coercivity Results}

Lemma 25 For every $\widetilde{v}=\left(\widetilde{v}_{1}, \widetilde{v}_{2}, \widetilde{v}_{3}\right) \in \mathcal{D}_{w}$ and every $\zeta \in \mathbb{R}^{4}$, one has

$$
\begin{aligned}
\int_{D}\left|\mathcal{E}_{D}(\widetilde{v})+\mathbf{M}_{\zeta}\right|^{2} d S_{2} d S_{3}= & \pi\left(\left|\zeta_{1}\right|^{2}+\frac{1}{8}\left(\left|\zeta_{3}\right|^{2}+\left|\zeta_{4}\right|^{2}\right)+\frac{1}{16}\left|\zeta_{2}\right|^{2}\right)+\frac{1}{4}\left\|\nabla \widetilde{v}_{1}\right\|_{L^{2}(D)}^{2} \\
& +\sum_{j, k=2}^{3}\left\|e_{j k}(\widetilde{v})\right\|_{L^{2}(D)}^{2}
\end{aligned}
$$


where $\mathbf{M}_{\zeta}=\left(\zeta_{1}+S_{3} \zeta_{3}-S_{2} \zeta_{4}\right) \mathbf{M}^{11}-S_{3} \mathbf{M}^{12}+S_{2} \mathbf{M}^{13}$.

Moreover, there exists a strictly positive constant $C$ such that

$$
|\zeta|^{2}+\|\widetilde{v}\|_{H^{1}(D)}^{2} \leq C \int_{D}\left|\mathcal{E}_{D}(\widetilde{v})+\mathbf{M}_{\zeta}\right|^{2} d S_{2} d S_{3} .
$$

Proof A direct calculation gives

$$
\begin{aligned}
\int_{D} & \left|\mathcal{E}_{D}(\widetilde{v})+\mathbf{M}_{\zeta}\right|^{2} d S_{2} d S_{3} \\
= & \int_{D}\left(\zeta_{1}+S_{3} \zeta_{3}-S_{2} \zeta_{4}\right)^{2} d S_{2} d S_{3}+\frac{1}{4} \int_{D}\left(\frac{\partial \widetilde{v}_{1}}{\partial S_{2}}-S_{3} \zeta_{2}\right)^{2} d S_{2} d S_{3} \\
& +\frac{1}{4} \int_{D}\left(\frac{\partial \widetilde{v}_{1}}{\partial S_{3}}+S_{2} \zeta_{2}\right)^{2} d S_{2} d S_{3}+\sum_{j, k=2}^{3}\left\|e_{j k}(\widetilde{v})\right\|_{L^{2}(\Omega)}^{2} .
\end{aligned}
$$

Observe that

$$
\int_{D}\left(-S_{3} \frac{\partial \widetilde{v}_{1}}{\partial S_{2}}+S_{2} \frac{\partial \widetilde{v}_{1}}{\partial S_{3}}\right) d S_{2} d S_{3}=0 .
$$

Hence, we obtain (G.1). Then (G.2) follows from the definition of $\mathcal{D}_{w}$, the PoincaréWirtinger and the Korn inequality.

Lemma 26 There exists a strictly positive constant $C$ such that

$$
\begin{aligned}
& \forall \mathcal{V} \in H_{\Gamma}^{1}(\Omega)^{3}, \quad \forall(\overline{\mathcal{V}}, \widehat{\mathcal{V}}, \widehat{\mathcal{B}}) \in L^{2}\left(\Omega ; \mathcal{D}_{E x} \times \mathcal{D}_{I n}\right), \quad \forall \widetilde{v} \in L^{2}\left(\Omega \times \mathcal{S} ; \mathcal{D}_{w}\right), \\
& \|\mathcal{V}\|_{H^{1}(\Omega)}^{2}+\|(\overline{\mathcal{V}}, \widehat{\mathcal{V}}, \widehat{\mathcal{B}})\|_{L^{2}\left(\Omega ; H^{1}(\mathcal{S})\right)}^{2}+\|\widetilde{v}\|_{L^{2}\left(\Omega ; H^{1}(D)\right)}^{2} \\
& \quad \leq C \int_{\Omega \times \mathcal{S} \times D}\left|\mathcal{E}(\mathcal{V})+\mathcal{E}_{\mathcal{S}}(\overline{\mathcal{V}}, \widehat{\mathcal{V}}, \widehat{\mathcal{B}})+\mathcal{E}_{D}(\widetilde{v})\right|^{2} d x d \widehat{S}
\end{aligned}
$$

Proof Step 1. A preliminary result.

Let $\zeta$ be a $3 \times 3$ symmetric matrix. Consider the displacements $\overline{\mathcal{W}} \in \mathcal{D}_{E x}$ and $\mathbb{V}_{\zeta}(x)=$ $\zeta x, x \in \mathbb{R}^{3}$. The restriction of $\mathbb{V}_{\zeta}$ to $\mathcal{S}$ belongs to $\mathbf{U}_{\mathcal{S}}$ and one has

$$
\frac{d \mathbb{V}_{\zeta}}{d S_{1}} \cdot \mathbf{t}_{1}=\left(\zeta \mathbf{t}_{1}\right) \cdot \mathbf{t}_{1}, \quad\left(\zeta \mathbf{t}_{1}\right) \cdot \mathbf{t}_{1}+\frac{\partial \overline{\mathcal{W}}}{\partial S_{1}} \cdot \mathbf{t}_{1}=\frac{\partial\left(\mathbb{V}_{\zeta}+\overline{\mathcal{W}}\right)}{\partial S_{1}} \cdot \mathbf{t}_{1}
$$

As in Step 2 of Lemma 16 and since the structure $\mathcal{S}$ is stable, we obtain a rigid displacement r such that

$$
\left\|\mathbb{V}_{\zeta}+\overline{\mathcal{W}}-\mathbf{r}\right\|_{H^{1}(\mathcal{S})} \leq C\left\|\frac{\partial\left(\mathbb{V}_{\zeta}+\overline{\mathcal{W}}\right)}{\partial S_{1}} \cdot \mathbf{t}_{1}\right\|_{L^{2}(\mathcal{S})} .
$$

Remind that $\mathcal{S}$ is also a 3-periodic structure. Therefore, comparing the values of $\mathbb{V}_{\zeta}+\overline{\mathcal{W}}-\mathbf{r}$ on the opposite faces of $Y \cap \mathcal{S}$ gives

$$
|\zeta-\mathbf{b}| \leq C\left\|\mathbb{V}_{\zeta}+\overline{\mathcal{W}}-\mathbf{r}\right\|_{H^{1}(\mathcal{S})},
$$

where $\mathbf{b}=\nabla \mathbf{r}$ is a $3 \times 3$ antisymmetric matrix. Hence,

$$
|\zeta|+|\mathbf{b}| \leq C\left\|\frac{\partial\left(\mathbb{V}_{\zeta}+\overline{\mathcal{W}}\right)}{\partial S_{1}} \cdot \mathbf{t}_{1}\right\|_{L^{2}(\mathcal{S})} .
$$


Since $\bar{W}$ belongs to $H_{p e r, 0}^{1}(\mathcal{S})^{3}$, we obtain

$$
|\zeta|+\|\overline{\mathcal{W}}\|_{H^{1}(\mathcal{S})} \leq C\left\|\frac{\partial\left(\mathbb{V}_{\zeta}+\overline{\mathcal{W}}\right)}{\partial S_{1}} \cdot \mathbf{t}_{1}\right\|_{L^{2}(\mathcal{S})} .
$$

Step 2. Inequality (G.2) leads to

$$
\begin{aligned}
& \int_{\Omega \times \mathcal{S}}\left|\left(e(\mathcal{V}) \mathbf{t}_{1}\right) \mathbf{t}_{1}+\frac{\partial \overline{\mathcal{V}}}{\partial S_{1}} \cdot \mathbf{t}_{1}\right|^{2} d \mathbf{S} d x+\|(\widehat{\mathcal{V}}, \widehat{\mathcal{B}})\|_{L^{2}\left(\Omega ; \mathcal{D}_{I n}\right)}^{2}+\|\widetilde{v}\|_{L^{2}\left(\Omega ; H^{1}(D)\right)}^{2} \\
& \quad \leq C \int_{\Omega \times \mathcal{S} \times D}\left|\mathcal{E}(\mathcal{V})+\mathcal{E}_{\mathcal{S}}(\overline{\mathcal{V}}, \widehat{\mathcal{V}}, \widehat{\mathcal{B}})+\mathcal{E}_{D}(\widetilde{\mathcal{V}})\right|^{2} d \widehat{S} d x
\end{aligned}
$$

Then, the estimate (G.4) and Lemma 16 give (G.3).

Lemma 27 There exists $C_{0}^{\prime}>0$ which does not depend on the variable $\mathbf{S}$, such that

$$
\forall \zeta \in \mathbb{R}^{4}, \quad \mathfrak{A} \zeta \cdot \zeta \geq C_{0}^{\prime}|\zeta|^{2} \quad \text { a.e. in } \mathcal{S} .
$$

Proof Set $\tilde{\chi}_{\zeta}=\sum_{q=1}^{4} \zeta_{q} \tilde{\chi}_{q}$. By (5.4) one obtains

$$
\mathfrak{A} \zeta \cdot \zeta \geq C_{0} \int_{D}\left|\mathcal{E}_{D}\left(\tilde{\chi}_{\zeta}\right)+\mathbf{M}_{\zeta}\right|^{2} d S_{2} d S_{3}, \quad \text { a.e. in } \mathcal{S}
$$

Lemma 25 yields $\int_{D}\left|\mathcal{E}_{D}\left(\tilde{\chi}_{\zeta}\right)+\mathbf{M}_{\zeta}\right|^{2} d S_{2} d S_{3} \geq \pi\left(\left|\zeta_{1}\right|^{2}+\frac{1}{8}\left(\left|\zeta_{3}\right|^{2}+\left|\zeta_{4}\right|^{2}\right)+\frac{1}{16}\left|\zeta_{2}\right|^{2}\right)$. Thus, (G.5) is proved.

\section{References}

1. Abdoul-Anziz, H., Seppecher, P., Bellis, C.: Homogenization of frame lattices leading to second gradient models coupling classical strain and strain-gradient terms. Math. Mech. Solids 24(12), 3976-3999 (2019)

2. Antman, S.S.: The theory of rods. In: Függe, S., Truesdell, C. (eds.) Handbuch der Physik, pp. 641-703. Springer, Berlin (1972)

3. Blanchard, D., Gaudiello, A., Griso, G.: Junction of a periodic family of elastic rods with a 3d plate. Part I. J. Math. Pures Appl. 88(1), 1-33 (2007)

4. Blanchard, D., Griso, G.: Asymptotic behavior of structures made of straight rods. J. Elast. 108(1), 85118 (2012)

5. Caillerie, D.: Thin elastic and periodic plates. Math. Models Methods Appl. Sci. 6(1), 159-191 (1984)

6. Casado-Díaz, J., Luna-Laynez, M., Martín, J.D., Gómez, J.D.: Homogenization of very thin elastic reticulated structures. J. Mech. Behav. Biomed. Mater. 16(4-5), 297-304 (2005)

7. Ciarlet, P.G.: Mathematical Elasticity II: Lower-Dimensional Theories of Plates and Rods. NorthHolland, Amsterdam (1990)

8. Cioranescu, D., Saint, J., Paulin, J.: Homogenization of Reticulated Structures. Applied Mathematical Sciences, vol. 136. Springer, New York (1999)

9. Cioranescu, D., Damlamian, A., Griso, G.: The periodic unfolding method in homogenization. SIAM J. Math. Anal. 40(4), 1585-1620 (2008)

10. Cioranescu, D., Damlamian, A., Griso, G.: The Periodic Unfolding Method: Theory and Applications to Partial Differential Problems. Springer, Berlin (2018)

11. Damlamian, A., Vogelius, M.: Homogenization limits of the equations of elasticity in thin domains. SIAM J. Math. Anal. 18(2), 435-451 (1987)

12. Geymonat, G., Krasucki, F., Marigo, J.J.: Sur la commutativité des passages à la limite en théorie asymptotique des poutres composites. C. R. Acad. Sci. Paris, Ser. I 305(2), 225-228 (1987) 
13. Griso, G.: Asymptotic behavior of curved rods by the unfolding method. Math. Methods Appl. Sci. 27(17), 2081-2110 (2004)

14. Griso, G.: Asymptotic behavior of structures made of curved rods. Anal. Appl. 6(1), 11-22 (2008)

15. Griso, G.: Decompositions of displacements of thin structures. J. Math. Pures Appl. 89, 199-223 (2008)

16. Griso, G., Khilkova, L., Orlik, J., Sivak, O.: Homogenization of perforated elastic structures. J. Elast. 141, 181-225 (2020). https://doi.org/10.1007/s10659-020-09781

17. Griso, G., Migunova, A., Orlik, J.: Asymptotic analysis for domains separated by a thin layer made of periodic vertical beams. J. Elast. 128(2), 291-331 (2017)

18. Griso, G., Miara, B.: Homogenization of periodically heterogeneous thin beams. Chin. Ann. Math., Ser. B 39(3), 397-426 (2018)

19. Griso, G., Orlik, J., Wackerle, S.: Homogenization of textiles. SIAM J. Math. Anal. 52(2), 1639-1689 (2020)

20. Griso, G., Orlik, J., Wackerle, S.: Asymptotic behavior for textiles in von-Kármán regime. J. Math. Pures Appl. 144, 164-193 (2020)

21. Griso, G., Hauck, M., Orlik, J.: Asymptotic analysis for periodic perforated shells. ESAIM: M2AN. https://doi.org/10.1051/m2an/2020067

22. Le Dret, H.: Modeling of the junction between two rods. J. Math. Pures Appl. 68(3) 365-397 (1989)

23. Le Dret, H.: Problèmes variationnels dans les multi-domaines. Modélisation des jonctions et applications Elsevier-Masson, Paris (1991)

24. Kolzlov, V., Maz'Ya, V., Mocvchan, A.: Asymptotic Analysis of Fields in Multi-Structures. Clarendon Press, Oxford (1999)

25. Martinsson, P.G., Babuška, I.: Homogenization of materials with periodic truss or frame microstructures. Math. Models Methods Appl. Sci. 17(5), 805-832 (2007)

26. Orlik, J., Panasenko, G., Shiryaev, V.: Optimization of textile-like materials via homogenization and dimension reduction. SIAM J. Multiscale Model. Simul. 14(2), 637-667 (2016)

27. Panasenko, G.: Asymptotic solutions of the system of elasticity theory for rod and frame structures. Russian Acad. Sci. Sb. Math. 75(1), 85-110 (1993)

28. Pastukhova, S.: Homogenization of problems of elasticity theory on periodic box and rod frames of critical thickness. J. Math. Sci. 130, 4954-5004 (2005)

29. Pilkey, W.: Analysis and Design of Elastic Beams, Computational Methods. Wiley, New York (2002)

30. Trabucho, L., Viano, J.M.: Mathematical Modeling of Rods. Handbook of Numerical Analysis, vol. 4. North-Holland, Amsterdam (1996)

31. Zhikov, V., Pastukhova, S.: Homogenization for elasticity problems on periodic networks of critical thickness. Sb. Math. 194(5), 61-96 (2003) 